
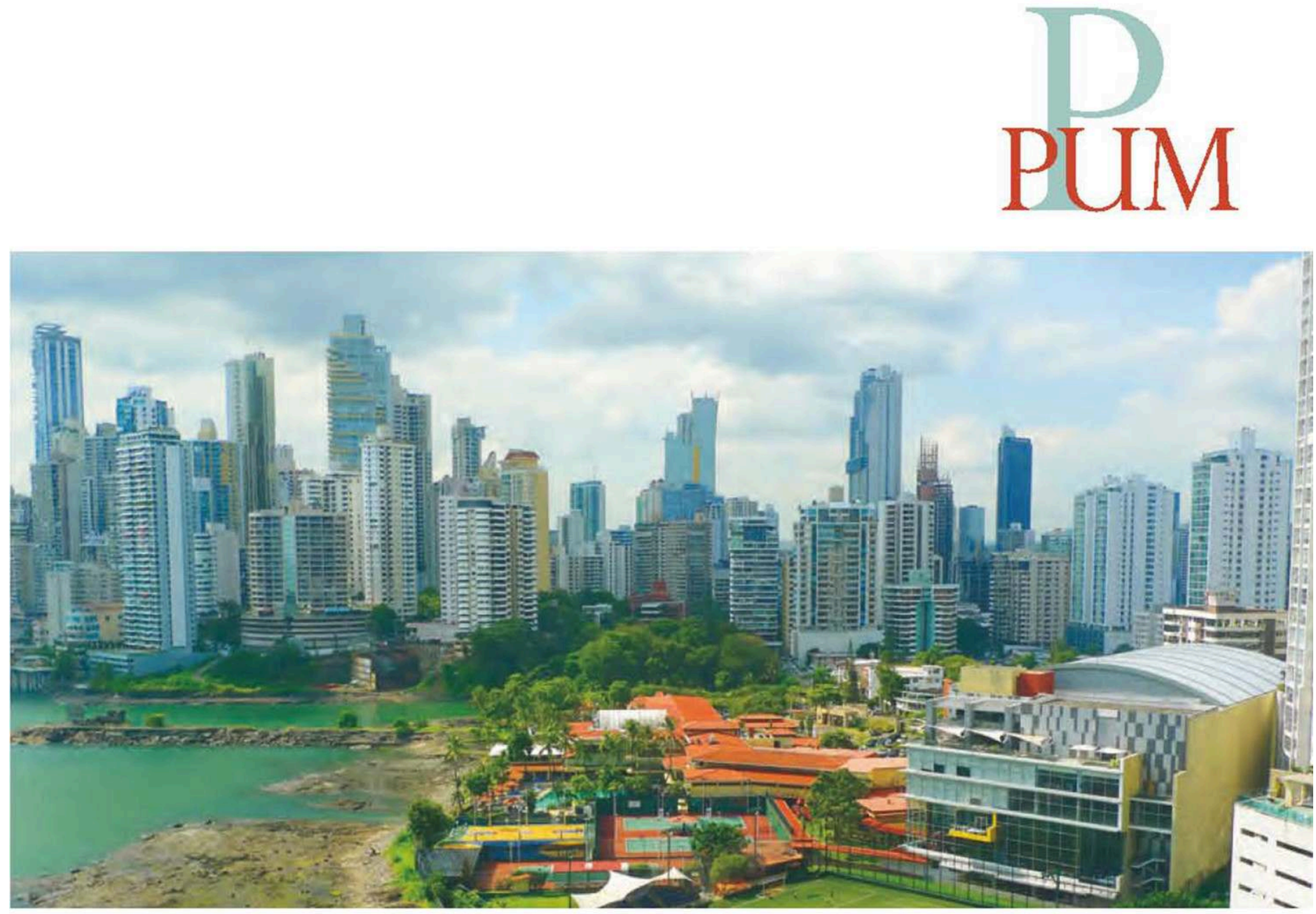

\title{
Les villes non occidentales
}

Comprendre les enjeux de la diversité urbaine

Sous la direction de

Gabriel Fauveaud 


\section{Les villes non occidentales}

Comprendre les enjeux de la diversité urbaine

\section{Gabriel Fauveaud (dir.)}

DOI : 10.4000/books.pum. 11857

Éditeur : Presses de l'Université de Montréal

Lieu d'édition : Montréal

Année d'édition : 2017

Date de mise en ligne : 18 mai 2018

Collection : PUM

EAN électronique : 9791036501845

\section{Q OpenEdition}

\section{Books}

https://books.openedition.org

\section{Édition imprimée}

EAN (Édition imprimée) : 9782760635142

Nombre de pages : 186

\section{Référence électronique}

FAUVEAUD, Gabriel (dir.). Les villes non occidentales : Comprendre les enjeux de la diversité urbaine. Nouvelle édition [en ligne]. Montréal : Presses de l'Université de Montréal, 2017 (généré le 29 janvier 2022). Disponible sur Internet : <http://books.openedition.org/pum/11857>. ISBN : 9791036501845 DOI : https://doi.org/10.4000/books.pum.11857. 


\section{RÉSUMÉS}

Cet ouvrage souhaite montrer la richesse d'une ouverture géographique et thématique des recherches urbaines actuelles en présentant celles qui prennent en considération les villes situées en dehors de l'aire occidentale. Il donne la voix à de jeunes chercheurs et praticiens originaires de six pays et de trois continents qui, par leurs réflexions engagées, proposent de corriger les perceptions souvent négatives, voire catastrophiques, de l'urbanisation non occidentale. En partant du principe que tout espace urbain est aussi banal que singulier, ils aident à décentrer le regard et à envisager sérieusement l'importance de l'altérité dans la construction des représentations collectives.

Ouvert sur le monde, donc, cet ouvrage montre tout l'intérêt de diversifier les approches théoriques et empiriques en urbanisme, dont le fort ancrage nord-américain ou européen empêche souvent de rendre compte des multiples réalités des villes de la planète.

\section{GABRIEL FAUVEAUD (DIR.)}

Docteur en géographie, Gabriel Fauveaud est chercheur invité au Centre d'études et de recherches international de l'Université de Montréal (CÉRIUM) et chercheur associé à l'UMR 8586 Prodig. 
Cet ouvrage est diffusé en accès ouvert dans le cadre du projet OpenEdition Books Select.

Ce programme de financement participatif, coordonné par OpenEdition en partenariat avec Knowledge Unlatched et le consortium Couperin, permet aux bibliothèques de contribuer à la libération de contenus provenant d'éditeurs majeurs dans le domaine des sciences humaines et sociales.

La liste des bibliothèques ayant contribué financièrement à la libération de cet ouvrage se trouve ici :

https://www.openedition.org/22515.

This book is published open access as part of the OpenEdition Books Select project.

This crowdfunding program is coordinated by OpenEdition in partnership with Knowledge Unlatched and the French library consortium Couperin. Thanks to the initiative, libraries can contribute to unlatch content from key publishers in the Humanities and Social Sciences.

Discover all the libraries that helped to make this book available open access: https://www.openedition.org/22515?lang=en.

\section{IAI Sopenedition coupernoren}



LES VILLES NON OCCIDENTALES 

Sous la direction de

Gabriel Fauveaud

\section{LES VILLES NON OCCIDENTALES}

COMPRENDRE LES ENJEUX DE LA DIVERSITÉ URBAINE 
Catalogage avant publication de Bibliothèque et Archives nationales du Québec et Bibliothèque et Archives Canada

Les villes non occidentales: comprendre les enjeux de la diversité urbaine

Comprend des références bibliographiques.

ISBN 978-2-7606-3514-2

1. Villes - Pays en voie de développement. 2. Urbanisme - Pays en voie de développement - Études de cas. I. Fauveaud, Gabriel.

HT149.5.V54 2017

307.7609172 '4

C2017-940552-7

Mise en pages: Folio infographie

ISBN (papier) : 978-2-7606-3514-2

ISBN (PDF) : 978-2-7606-3515-9

ISBN (ePub) : 978-2-7606-3516-6

Dépôt légal: $2^{\mathrm{e}}$ trimestre 2017

Bibliothèque et Archives nationales du Québec

(C) Les Presses de l'Université de Montréal, 2017

Les Presses de l'Université de Montréal remercient de leur soutien financier le Conseil des arts du Canada et la Société de développement des entreprises culturelles du Québec (SODEC).

Finance parl gouvernement du canada $\mid$ Canadà' 


\section{Remerciements}

Cet ouvrage, fruit d'un véritable travail collectif, a été rédigé à partir de discussions tenues au cours d'un atelier de travail en juin 2014 à l'Université de Montréal. Il n'aurait pas vu le jour sans l'implication active de tous les participants, avant, pendant et après l'atelier. Nous remercions tous les auteurs de s'être prêtés au jeu de la critique et de la coproduction scientifique.

Merci par ailleurs à Hélène Bélanger, professeure au Département d'études urbaines de l'Université du Québec à Montréal, et à Michel-Max Raynaud, professeur agrégé à l'Institut d'urbanisme de l'Université de Montréal, qui ont animé l'atelier; cet ouvrage est aussi nourri de leurs commentaires, de leurs remarques et de leurs synthèses.

L'atelier de travail destiné à la rencontre de jeunes chercheurs en études urbaines est, à l'origine, une idée menée par Franck Scherrer, directeur de l'Institut d'urbanisme de l'Université de Montréal, par l'intermédiaire de l'Association pour la promotion de l'enseignement et de la recherche en aménagement et urbanisme (APERAU). Nous le remercions ici chaleureusement de son initiative.

Latelier de travail n'aurait pu avoir lieu sans soutien financier, tant pour le déroulement de l'événement que pour la venue des chercheurs. Nous remercions à ce titre le réseau interuniversitaire Villes Régions Monde, l'Institut d'urbanisme de l'Université de Montréal, l'Observatoire Ivanhoé Cambridge du développement urbain et 
immobilier de la Faculté de l'aménagement de l'Université de Montréal, et l'APERAU.

Enfin, la publication de cet ouvrage a reçu le soutien financier de l'APERAU et de l'Association internationale des maires francophones, que nous remercions également. 


\section{Avant-propos}

Les auteurs de cet ouvrage constituent le Collectif de recherche pour un urbanisme ouvert sur les mondes (CORUM), regroupement à vocation pluridisciplinaire formé en 2014 et composé de jeunes chercheurs et praticiens. Il est parrainé par l'APERAU. La publication de cet ouvrage répond à un double constat que partagent les membres du CORUM à propos des villes non occidentales (expression sur laquelle nous reviendrons) : 1) elles restent faiblement représentées au sein des recherches urbaines francophones; 2) il existe un réel besoin de diversification des approches théoriques et méthodologiques permettant de mieux rendre compte de la diversité du phénomène urbain dans le monde. Cet ouvrage veut en ce sens ouvrir un espace de réflexion et de discussion en faveur d'une plus grande considération des villes non occidentales dans la production des savoirs en recherches urbaines.

Afin de répondre à cet objectif, l'ouvrage donne la voix à de jeunes chercheurs et praticiens non occidentaux et occidentaux, qui ont tous étudié au sein d'institutions du Nord et qui travaillent actuellement dans des universités, des institutions ou des entreprises localisées en Afrique du Nord, en Afrique subsaharienne, en Amérique latine, en Amérique du Nord ou en Europe de l'Ouest.

Par ailleurs, le positionnement éditorial de l'ouvrage s'inscrit dans une volonté d'amener les étudiants et les jeunes chercheurs à accorder 
une attention plus manifeste aux recherches urbaines des villes non occidentales. En conséquence, plutôt que de centrer leurs propos sur des résultats de recherche comme des contributions à un ouvrage scientifique classique l'auraient fait, les auteurs ont choisi d'insister sur leurs questionnements de recherche et de proposer des points de vue originaux sur les aspects pratiques et réflexifs de leurs activités de chercheurs ou de professionnels. Les chapitres sont donc courts et comportent peu de références bibliographiques. Nous espérons que ce positionnement permettra de mieux faire connaître certains enjeux des recherches urbaines au sein des villes non occidentales auprès des étudiants, des jeunes chercheurs et des professionnels de l'urbain. L'ouvrage peut ainsi être vu comme un guide pratique avant d'aller sur le terrain, pour des étudiants commençant à travailler sur des villes non occidentales, ou encore comme un recueil de témoignages révélateur de certaines interrogations théoriques et méthodologiques que se posent les chercheurs et praticiens du phénomène urbain. 


\title{
Repenser la place des villes non occidentales dans les recherches urbaines
}

\author{
Gabriel Fauveaud
}

L'ancrage occidental historique de la recherche urbaine et l'attention privilégiée portée aux villes européennes et nord-américaines obligent à remettre en question la capacité des cadres théoriques et méthodologiques constitués à rendre compte de la diversité entre les réalités urbaines. ${ }^{1}$. Si les différences de trajectoires urbaines empruntées par les villes occidentales donnent lieu à de nombreux débats et, dans une certaine mesure, à un important travail épistémologique, les villes situées en dehors du monde occidental restent faiblement mobilisées dans les réflexions portant sur l'évolution des cadres théoriques et méthodologiques de la recherche urbaine. Pourtant, ces villes font l'objet d'une attention croissante depuis les trente dernières années. Le poids grandissant des économies émergentes dans la globalisation, l'accélération des rythmes d'urbanisation, ou encore la mondialisation accrue des processus urbanistiques ont certainement participé au renouvellement des recherches urbaines en dehors du monde occi-

1. Je souhaiterais remercier chaleureusement Martine Drozdz et Juliette Maulat d'avoir lu attentivement et commenté consciencieusement une première version de cette introduction. Par ailleurs, l'approche éditoriale privilégiée pour cet ouvrage a été de limiter la citation d'auteurs et de travaux scientifiques. J'invite donc le lecteur à consulter la bibliographie générale pour prendre connaissance de l'ensemble des travaux ayant servi à la rédaction de cette introduction. 
dental, particulièrement manifeste depuis les années 2000. On sent alors un important décalage entre une production scientifique très structurée par l'expérience occidentale et des recherches urbaines de plus en plus nourries par d'autres logiques d'urbanisation, d'autres trajectoires urbaines et d'autres réalités citadines.

Face à ce constat, le qualificatif «non occidental» que des géographes et des urbanistes, tant anglophones que francophones, proposent d'employer (voir notamment Lévy, 1997; Edensor et Mark, 2012) sert de plaidoyer en faveur d'une plus grande ouverture et diversification géographiques des approches empiriques et théoriques de la recherche urbaine. Ce terme vise aussi à dépasser les tropismes géographiques (les villes du Sud) et économiques (les villes en développement) qui caractérisent les appellations couramment utilisées pour dénommer ces espaces urbains. Bien entendu, le terme «non occidental» remplace un tropisme par un autre, celui de l'aire civilisationnelle, qui renvoie à une représentation du monde tout aussi subjective et discutable. Cependant, il vise justement à souligner la prévalence d'approches occidentalo-centrées des dynamiques urbaines dans le monde, conséquence de la polarisation de la production scientifique au sein des pays occidentaux et de la domination des cadres théoriques forgés en Occident, généralement à partir de l'étude de villes occidentales. L'idée tenace que les villes non occidentales ne tendraient qu'à converger vers les occidentales, ou que le phénomène urbain est universel puisqu'il ne représente que l'expression d'un système de production devenu global, en sont quelques exemples parmi tant d'autres.

$\mathrm{Au}$ sein du monde francophone, les villes non occidentales ne semblent pas composer un champ de recherche spécifique. On privilégie souvent les approches thématiques, parfois dans une perspective très descriptive, où il est par exemple question de transitions démographiques, de dynamiques résidentielles, de politiques urbaines, d'accès aux services, ou encore d'informalité. En conséquence, de nombreux travaux abordent les villes non occidentales selon des modèles, des typologies et des catégorisations bien ancrés dans la 
culture occidentale et ses formes particulières de rationalité, sans pour autant examiner de manière plus systématique les fondements méthodologiques et théoriques qui déterminent leurs approches scientifiques. Pourtant, une approche détaillée de la littérature francophone témoigne de l'existence de réflexions épistémologiques riches (portant tant sur les conditions de la production des savoirs que sur leurs fondements conceptuels) attachées aux villes non occidentales. Les positionnements théoriques et méthodologiques existent donc, mais ils semblent dispersés dans la production scientifique. Plusieurs interrogations émergent: quelles sont les spécificités des approches théoriques et empiriques portant sur les villes non occidentales? L'évolution de la recherche urbaine sur ces villes est-elle spécifique, ou correspondelle au contraire à l'évolution plus générale de la discipline? Plus largement, les travaux sur les villes non occidentales constituent-ils un champ de recherche particulier?

Cette introduction propose de dresser un état intermédiaire des connaissances, des programmes, des pratiques et des positionnements scientifiques - voire de certaines postures éthiques - propres aux villes non occidentales. Cela permettra de mieux identifier certaines caractéristiques des recherches urbaines sur ces villes. La dernière partie de l'introduction émettra quelques propositions en faveur d'un décentrement géographique, théorique et méthodologique des recherches urbaines francophones où les villes non occidentales pourraient jouer un rôle de premier plan.

\section{Un champ de recherche spécifique dans le monde francophone?}

L'évolution des recherches urbaines francophones sur les villes non occidentales depuis les années 1980-1990 s'est peu accompagnée, en tout cas de manière explicite et assumée, d'un travail réflexif global examinant les courants théoriques, les concepts, les méthodes, voire les positionnements politiques et professionnels structurant la production des savoirs. Pourtant, une revue de la littérature témoigne de la richesse des réflexions théoriques et empiriques sur ces espaces, 
qu'il faut par ailleurs considérer dans la perspective de l'évolution des sciences sociales depuis les années 1970-1980.

\section{De villes du Tiers-Monde à villes non occidentales}

Le passage de l'appellation «villes du Tiers-Monde» à «villes du Sud» au cours des années 1980-1990 témoigne d'une évolution des approches scientifiques et techniques des chercheurs et praticiens vis-à-vis des villes non occidentales. Si les recherches dans les années 1970-1980 sont largement marquées par des approches centrées sur le mal-développement et les relations centres-périphéries, à partir des années 1990-2000, elles s'inscrivent tant dans la réorganisation des dynamiques économiques et politiques, avec la fin de la guerre froide, que dans l'accélération des dynamiques de mondialisation et de globalisation.

Une partie de ces travaux s'inscrit toutefois dans la continuité des recherches urbaines des années 1970-1980. On peut ainsi noter la prégnance d'une vision alarmiste et catastrophique du développement urbain, où «l'explosion urbaine» et les métropoles qui «enflent et qui laminent» sont encore perçues comme les principaux dangers du «sous-développement» (Troin, 2000: 148). L'idée selon laquelle la ville reste un véritable défi pour les pays du Sud est encore partagée par certains acteurs de la coopération internationale. L'importance des questionnements relatifs à la tradition et à la modernité, en droite ligne avec les approches développementalistes traitant des transformations sociospatiales des pays du Sud, témoigne de l'héritage encore prégnant des approches tropicalistes et développementalistes du monde francophone. Une partie de la littérature présente ainsi un jugement particulièrement pessimiste des trajectoires de développement dans les pays du Sud, où le fait urbain est autant une cause qu'un révélateur de ces évolutions.

En parallèle, au cours des années 1990, la place de la ville et de l'urbanisation dans les dynamiques de développement au Sud change progressivement. L'évolution des discours et des registres d'action des grandes agences internationales de développement, qui considèrent 
depuis les années 1990 la croissance urbaine comme une occasion de développement plutôt que comme un frein à celui-ci, a certainement favorisé l'abandon progressif des visions et des discours négatifs sur les villes non occidentales. Le fait urbain est de moins en moins perçu comme un défi, et plutôt étudié comme un révélateur ou une cause de changements sociospatiaux plus profonds du Sud global. Les conséquences de l'accélération de l'urbanisation sont de plus en plus étudiées de manière thématique. Les chercheurs s'intéressent ainsi à l'évolution des pratiques et des dynamiques foncières, aux politiques d'aménagement du territoire, aux nouvelles relations entre villes et campagnes, ou encore à l'adaptation des stratégies résidentielles. De même, on considère de plus en plus les villes comme situées au cœur d'évolutions sociétales plus larges, qui doivent aussi se rapporter aux échelles nationales.

La libéralisation accrue des logiques de production de la ville devient aussi un sujet de première importance. Des approches critiques plus diversifiées, portant sur les nouvelles logiques de développement urbain et sur les processus d'exclusion et de marginalisation sociospatiales leur correspondant, remplacent peu à peu les approches marxistes des années 1960-1980 sur le Tiers-Monde. Les approches sectorielles ou thématiques sont favorisées, pour montrer comment les nouvelles logiques de marché transforment les dynamiques de production et de gestion des espaces urbains, que ce soit à grande ou à petite échelle. De nombreux auteurs mettent aussi en avant une plus grande transversalité thématique. Par exemple, l'étude de la libéralisation de la production de l'habitat et du désengagement des États dans le financement de l'accès au logement amène à considérer conjointement les statuts d'occupation du sol, les pratiques citadines, les modes d'habiter et les pratiques résidentielles (Bertrand, 2003).

Dans ce cadre d'analyse, les politiques urbaines des grandes agences internationales sont passées au crible, tout comme leurs discours et leurs registres d'action, comme le révèlent les critiques portées aux notions de bonne gouvernance ou de best practices. Les chercheurs s'interrogent également sur la capacité des politiques de 
développement à véritablement inclure les populations les plus précaires dans les processus décisionnels. Les objectifs de rentabilité, de croissance et de plus-value, qui orientent de manière plus explicite les politiques de développement des acteurs gouvernementaux et non gouvernementaux, sont souvent accusés de nuire à la mise en œuvre d'approches plus inclusives du développement urbain.

Si ces recherches ne s'inscrivent généralement pas de manière explicite au sein de courants théoriques précis, certains auteurs mentionnent les nouveaux enjeux réflexifs, disciplinaires et épistémologiques soulevés par l'évolution des logiques de production des villes non occidentales (privatisation, décentralisation, développement par projets, etc.). Sans rupture manifeste avec le paradigme modernité/ développement qui structure les recherches urbaines des années 19701980, la recherche urbaine des années 1990-2000 examine parfois la capacité des cadres théoriques mobilisés à rendre compte des changements urbains à l'œuvre depuis les années 1980, avec un net souci de réfléchir aux conditions de la production des connaissances. Monique Bertrand insiste sur ce point à propos des villes du Sud et d'Afrique de l'Ouest:

Cette théorie plus générale se cherche depuis la confrontation des pensées marxistes, néo-libérales et tiers-mondistes des années 1970. [...] Elle renvoie aux conditions d'élaboration des connaissances - et à la relation particulière que les chercheurs du Nord et du Sud entretiennent avec leurs propres contextes académiques, milieux intellectuels et carcans cognitifs. Il paraît donc nécessaire d'objectiver plus que jamais tous les moments de ce positionnement en ricochet, sans perdre prise avec des terrains précis d'investigation (1998: 901).

Nous souscrivons tout à fait à ce constat et au souhait de mieux objectiver la production des savoirs urbains à propos des villes non occidentales. Pour ce faire, il apparaît indispensable d'effectuer un double travail réflexif, tant sur les logiques de production de la connaissance que sur les savoirs produits. Cela semble d'autant plus nécessaire qu'un décalage apparaît entre l'évolution des dynamiques urbaines et celle des cadres conceptuels mobilisés pour les comprendre. Ces der- 
niers procèdent certes d'un recadrage thématique et scalaire depuis les années 1990, sans pour autant être accompagnés d'une approche réflexive de fond. Ceux-ci restent trop souvent tributaires de typologies spatiales, de conceptions urbanistiques ou d'appréciations des pratiques citadines forgées par des chercheurs occidentaux ou au sein de contextes universitaires et professionnels du Nord. Ils peinent donc parfois à proposer des approches et des méthodes adaptées aux conditions spécifiques et localisées de l'urbanisation. Pourtant, les appels à un décentrement de la recherche urbaine sont nombreux. Depuis les années 1990, mais surtout à partir des années 2000, nous assistons à une remise en cause beaucoup plus manifeste des approches et des concepts traditionnellement mobilisés pour l'étude des villes non occidentales. Les critiques portent, par exemple, sur la manière d'aborder l'informalité des pratiques économiques, sur les écueils de la pensée occidentale rationaliste, ou encore sur la nécessité de repenser l'habitat spontané.

En ce sens, on remet en question la domination des approches duales des dynamiques urbaines au Sud (formel/informel, planifié/ non planifié, réglementé/spontané, légal/illégal, etc.) qui prévalait jusqu'alors, et dans son sillage la notion même de développement. À ce titre, le tournant global des recherches urbaines a joué un rôle important dans l'évolution des approches sur les villes non occidentales, parfois de manière paradoxale.

\section{Le tournant global des recherches urbaines}

L'évolution des recherches urbaines francophones à propos des villes non occidentales est largement tributaire du tournant global des sciences sociales qui s'opère dès la fin des années 1980. Si le terme "global» se formalise dans les sciences sociales dès la deuxième moitié $\mathrm{du} \mathrm{XX}^{\mathrm{e}}$ siècle, c'est véritablement au cours des années 1980 que les approches globales dans les sciences sociales se popularisent, notamment à partir des travaux menés dans le monde universitaire anglophone (Steger, 2010). Les recherches portant sur les villes mondiales 
et globales, ainsi que le rôle des villes dans l'émergence d'une société en réseau global (Castells, 1999), ont largement privilégié une ouverture géographique des approches du fait urbain. Pour les recherches urbaines, les global studies apparaissent d'autant plus importantes que le monde devient, au début du $\mathrm{XXI}^{\mathrm{e}}$ siècle et pour la première fois de son histoire, majoritairement urbain. L'une des représentations communes entre un Nord industrialisé et urbanisé et un Sud sous-développé et principalement rural est donc en train de s'effriter, ou en tout cas de changer de nature.

L'émergence de grandes villes et de régions urbaines en dehors des pays occidentaux fait l'objet d'un nombre toujours croissant de travaux depuis les années 1980, que ce soit au sein de régions particulières ou à l'échelle globale. Dans les recherches urbaines, l'attrait pour ces très grands ensembles urbains procède de différents facteurs. Une partie des travaux s'inscrit dans la continuité directe des théories de la ville globale et cherche à repenser la place des villes non occidentales dans la hiérarchie métropolitaine mondiale. Il s'agit ainsi de cerner différentes formes et processus de métropolisation en Asie, en Afrique ou en Amérique latine.

Si le tournant global des recherches urbaines a très certainement participé à leur ouverture géographique, l'importance accordée aux grandes métropoles et aux régions urbaines, ainsi qu'aux pays et aux villes les plus intégrés à la globalisation et à la mondialisation, a automatiquement favorisé une polarisation des recherches vers les pays considérés comme émergents. On voit donc se multiplier, depuis les années 1990, des travaux portant sur la Chine, l'Inde, le Brésil, le Mexique ou encore l'Afrique du Sud, laissant apparaître de manière encore plus manifeste les angles morts de la recherche urbaine sur les villes non occidentales. Par ailleurs, les approches conceptuelles et méthodologiques employées correspondent bien souvent à celles mobilisées dans l'étude des villes occidentales, ce qui pose un grand nombre de questions méthodologiques. Particulièrement au sein des pays considérés comme émergents, l'approche des dynamiques urbaines s'inscrit explicitement ou implicitement dans la trajectoire 
présupposée du développement économique, et donc de l'expérience occidentale (industrialisation, métropolisation, intégration internationale, etc.). Pour de nombreux chercheurs, plus les villes se développent, plus elles convergent vers différents modèles de la ville occidentale, phénomène que la globalisation économique et urbanistique accentuerait.

Les auteurs d'un ouvrage dirigé par Dominique Lorrain (2011) proposent une analyse des modes de gestion des réseaux et des infrastructures, ainsi que des logiques de gouvernement, dans ce qu'ils nomment les «métropoles XXL en pays émergents». Dans l'introduction, Lorrain annonce une volonté de dépasser la pensée dominante selon laquelle les très grandes villes du Sud sont chaotiques et ingouvernables, pour montrer que d'autres logiques de régulation sont à l'œuvre. L'auteur revient sur la trajectoire particulière des villes des pays en développement, dont les évolutions ne peuvent être pensées selon la même grille d'analyse que celle mobilisée pour l'étude des villes des pays industriels:

En résumé, sur plusieurs facteurs structurants - le régime politique, l'organisation de l'espace, le modèle de services urbains -, les métropoles émergentes XXL apportent des réponses non habituelles aux défis urbains. Elles invitent à une étude spécifique qui les pense comme des mondes en soi qu'il s'agit d'investiguer dans plusieurs dimensions. [...] À trop s'intéresser aux villes globales, à faire de Londres, New York, Tokyo des modèles, on finit par produire une image déformée du monde (2011: 25-33).

On pourrait tout à fait appliquer le même constat à des villes qui ne sont ni globales, ni des métropoles émergentes. La hiérarchie métropolitaine imposée par la compétition interurbaine globale, tout comme le positivisme développementaliste, ne produisent pas seulement une image déformée du monde: ils structurent les méthodes, les sujets et les terrains de recherche, généralement loin de l'ordinaire des réalités citadines locales.

Pourtant, avec le virage global s'opère un changement de fond dans les échelles d'analyse mobilisées et les thématiques abordées. D’une 
part, le global remet sur le devant de la scène le local; d'autre part, il accompagne une évolution des approches thématiques du fait urbain dans les villes non occidentales.

\section{Un repositionnement progressif et généralisé des recherches}

L'extension des processus de mondialisation et de globalisation économique a largement participé à brouiller les catégories Nord-Sud, notamment avec l'émergence de grandes métropoles et de mégalopoles internationales dans les pays en développement et émergents, ou encore par la diffusion de formes urbaines internationalisées, comme les communautés fermées. L'adaptation des recherches urbaines découle d'une évolution des approches et des méthodes, d'une diversification des thèmes de recherche et d'une transversalité thématique et scalaire plus manifeste.

Tout d'abord, les approches comparatives Nord-Sud et Sud-Sud deviennent plus courantes. L'objectif consiste notamment à mieux comprendre des dynamiques urbaines touchant l'ensemble des villes dans le monde. Pour Koop et Amilhat (2011) par exemple, dépasser le paradigme Nord-Sud permet de souligner que les logiques de production de la ville et leurs discours techniques et politiques tendent à s'universaliser sous l'effet d'une intégration généralisée des territoires aux réseaux et flux de la globalisation économique. Dans ce contexte, certains concepts, comme la fragmentation urbaine ou le droit à la ville, font l'objet de comparaisons internationales et d'analyse transversales. Cependant, et nous y reviendrons, il faut être attentif à ne pas confondre internationalisation et universalisation, ce dernier terme sous-entendant parfois une homogénéisation globale de la fabrique urbaine.

La globalisation du fait urbain pousse aussi à reconsidérer des dynamiques spatiales spécifiques. À propos du processus de périurbanisation, par exemple, de nombreux auteurs soulignent le foisonnement de concepts cherchant à mieux définir ce processus de croissance urbaine en fonction des réalités territoriales locales. Dans 
un ouvrage récent de 2014, fruit du programme de recherche PERISUD, Jean-Louis Chaléard insiste ainsi sur la nécessité d’adopter des approches comparatives, notamment pour mieux saisir les particularités locales et régionales de la périurbanisation. Considérer ce phénomène de manière globale permet ainsi de mieux cerner des logiques sociospatiales locales de plus en plus diversifiées. La question du rapport entre développement périurbain et métropolisation au sein des capitales ou d'importantes métropoles émergentes se situe au cour de l'analyse. Le positionnement théorique de l'ouvrage, notamment articulé autour de l'idée de relation, témoigne d'une volonté de mieux caractériser ces espaces d'entre-deux, qui brouillent les catégories traditionnelles, rurales et urbaines. Dans ce contexte, les approches régionales et locales s'avèrent non seulement fécondes, mais aussi primordiales.

L'accélération globale du fait urbain s'est par ailleurs largement traduite par une attention accrue portée aux logiques de circulation, à la fois d'hommes, de capitaux et d'influences urbanistiques. Il s'agit de comprendre comment circulent les modèles urbains au Sud ou au sein d'espaces régionaux spécifiques, notamment par l'intermédiaire des pratiques professionnelles. La recherche s'intéresse aussi à la réorganisation des logiques de gouvernance, en lien avec la diffusion internationale de nouvelles formes urbaines et la généralisation d'un urbanisme de projet (Barthel, 2006; Goldblum, 2012). De même, on étudie de près les rapports de pouvoir conditionnant les modes de gestion politiques et techniques de l'urbanisation. Les chercheurs mettent souvent en valeur les tactiques et les stratégies d'adaptation des gouvernants locaux, qui reformulent les cadres normatifs de la gestion urbaine (décentralisation, partenariats publics-privés, gouvernance, etc.) en fonction de la réalité des rapports de force locaux. L'intérêt pour les logiques de circulation et de mobilité touche aussi le champ des migrations, que ce soit à l'échelle locale ou internationale. Les nouvelles logiques de mobilité impliquent tant l'émergence de formes inédites d'urbanité et de cosmopolitisme (Varrel, 2013) que de nouvelles logiques de production urbaine. 
L'évolution thématique des recherches urbaines dans les villes non occidentales est donc particulièrement tributaire des nouvelles articulations du rapport global/local. De manière croissante, de nombreux auteurs considèrent que les modèles internationaux et leurs circulations ne peuvent être étudiés sans la prise en compte, à l'échelle locale, des modes de gouvernance, des trajectoires urbaines, des dynamiques sociospatiales, des discours et des représentations, ou encore des logiques d'appropriation de l'espace, qui participent de la diversité des logiques d'urbanisation. Au croisement de la géographie urbaine, de l'aménagement du territoire et de l'urbanisme, les chercheurs insistent sur les processus de territorialisation et de reterritorialisation des modes de fabrication de l'urbain. Nous retrouvons de tels positionnements sur la question des transports par exemple. Il ne s'agit plus seulement d'étudier le rôle des infrastructures dans le charpentage des territoires, mais aussi de considérer le transport comme un lieu, producteur et constitué de relations, impliquant représentations identitaires et appropriations sociospatiales (Lombard et Steck, 2004). On rattache ainsi le transport à un fait citadin, à la fois dans ses dimensions urbanistiques, sociospatiales et politiques.

Au-delà du local, le tournant global a aussi amené une certaine résurgence des approches régionales. De nombreux travaux veulent montrer en quoi telle ou telle région du monde prend part à la globalisation du phénomène urbain de manière originale, dans la continuité de logiques citadines et urbaines en contexte local. En Asie, on s'interroge sur les trajectoires historiques spécifiques des villes d'Asie du Sud en lien avec la libéralisation économique et les particularismes des dynamiques sociales du sous-continent. De même, certains chercheurs, comme Franck, Goldblum et Taillard, évoquent les différentes expressions territoriales de la modernisation urbaine asiatique, ou encore les logiques de «métropolisation secondaire » à l'échelle sudest asiatique (2012).

Une transversalité scalaire et thématique plus importante accompagne ainsi la diversification des méthodes, des approches et des terrains de recherche. L'attention accrue portée aux processus plutôt 
qu'aux portraits thématiques ou monographiques s'étend aussi à l'évolution des discours et des positionnements des chercheurs et des praticiens. Dansereau et Navez-Bouchanine (2002) remarquent à cet égard que l'étude des dynamiques résidentielles des villes en développement depuis les années 1970 procède de deux postures dominantes: d'un côté, un discours de l'intervention, qui explique comment l'habitat devrait être; de l'autre, un discours critique, qui s'attache principalement à rendre compte des inégalités. Les auteures insistent sur la portée normative de tels positionnements, qui rendent peu compte de la diversité des réalités habitantes, et soulignent le dépassement nécessaire des présupposés normatifs occidentaux, comme la critique du clientélisme ou les présumées vertus de la démocratie participative. Elles en appellent à une meilleure complémentarité entre recherches et actions pour orienter la décision politique dans l'aménagement et l'urbanisme.

L'évolution de la production scientifique illustre doncl'importance que l'on donne à la connaissance du terrain et aux travaux empiriques, indispensables au renouvellement des recherches. La généralisation des approches multidisciplinaires et transdisciplinaires en géographie et en urbanisme, tout comme l'insistance plus manifeste sur les dynamiques sociales des transitions urbaines, doit aussi être resituée dans le «tournant spatial» des sciences sociales (Lévy, 1999). Pourtant, peu de travaux francophones proposent explicitement de réfléchir aux conditions de la production des savoirs sur les villes non occidentales, que ce soit d'un point de vue méthodologique ou théorique. Un constat partagé et bien souvent implicite semble donc s'imposer: la connaissance du terrain et le travail empirique amèneront d'eux-mêmes une réflexion théorique propre à rendre compte des réalités urbaines locales. Une telle posture peut cependant présenter des limites: ne pas faire face de manière explicite aux conditions de la production des connaissances limite la capacité des cadres conceptuels à intégrer, à déconstruire ou tout simplement à considérer le caractère normatif des postures et des présupposés du producteur de savoirs, des disciplines scientifiques et des contextes professionnels. 
Cela étant dit, la montée en force et la diversification des approches critiques ont largement participé à faire évoluer les cadres théoriques et méthodologiques de la recherche urbaine. Si les villes du Nord ont été au cœur de ce renouvellement dès les années 1990, les villes non occidentales y occupent une place de plus en plus centrale depuis les années 2000.

\section{Le rôle central du renouvellement des recherches critiques}

La diversification des échelles d'analyse et le renouvellement des approches et des thématiques accompagnent une relativisation progressive du caractère global des processus d'urbanisation. Pour beaucoup d'auteurs, si l'urbanisation est bien un phénomène planétaire, elle ne peut être considérée comme un processus universel, qui conduirait à l'homogénéisation de la fabrique urbaine et des pratiques citadines. Les monographies urbaines tendent à s'éclipser au profit d'études portant sur des populations, des groupes sociaux ou des processus spécifiques, mais aussi sur des espaces urbains plus modestes. L'analyse des pratiques, des discours et des représentations y occupe souvent une place de choix. Dans ce contexte, les réflexions thématiques s'appuient de manière croissante sur des recherches relevant de l'ethnographie, tant dans leurs méthodes que dans leur rapport aux objets étudiés. Les réflexions portant sur les cadres théoriques et méthodologiques mobilisés, ainsi que sur les objets ou les sujets étudiés, accompagnent souvent la production d'un savoir plus localisé. Le renouvellement des recherches critiques joue ici un rôle central.

Tout d'abord, de nombreux travaux témoignent d'un positionnement plus assumé dans le champ des études urbaines, bien souvent favorisé par une mobilisation de la littérature anglophone. À la croisée de l'économie politique et de l'urbanisme, on s'intéresse par exemple aux effets de la décentralisation, à la généralisation des partenariats publics-privés et à la diffusion de nouvelles formes de marchandisation des services. À ce titre, Baron et Peyroux proposent une approche 
théorique de la néolibéralisation des services urbains, en centrant leurs analyses sur l'évolution des formes de partenariats publicsprivés et leurs applications concrètes au sein de deux contextes africains. Lapproche théorique permet aux auteures de préconiser un déplacement des échelles d'analyse, afin de:

dépasser des théories économiques qui se focalisent sur les outils de la coordination (nature du contrat, modes de gouvernance les plus efficaces) pour privilégier, à l'instar d'approches en économie politique, sciences politiques et géographie, des analyses qui intègrent les facteurs sociopolitiques et institutionnels en considérant à part entière la dimension du pouvoir et les rapports de force, comme le proposent également certaines théories institutionnalistes (2011).

Le renouvellement des approches critiques amène à repenser certains thèmes déjà populaires au sein des recherches urbaines sur les villes du Tiers-Monde, comme les logiques politico-économiques de la production de l'urbain. Les recherches s'intéressent aux effets du néolibéralisme, à la précarisation de l'habitat informel sous la pression de la spéculation foncière, ou encore à l'ouverture des marchés aux promoteurs et acteurs privés. On étudie aussi la montée en puissance des acteurs privés, leur prise en main des logiques de production de la ville, tout comme l'extrême volatilité de marchés immobiliers principalement dédiés à la promotion privée. En privilégiant des approches ethnographiques, les recherches critiques proposent ainsi de repenser certains thèmes centraux des recherches urbaines dans les villes non occidentales, comme l'informalité par les logiques d'éviction foncière ou d'éradication des quartiers précaires. Il s'agit alors de redéfinir certaines notions centrales liées à l'exclusion de certains habitants ou groupes sociaux, comme les marges, ou alors de mobiliser des cadres conceptuels nouveaux pour les villes des pays en développement, comme celui de «justice spatiale» (GervaisLambony et al., 2014).

Cependant, malgré cette évolution généralisée des cadres théoriques et méthodologiques, un important effort de reconceptualisation des dynamiques urbaines en dehors des pays occidentaux reste 
à accomplir. Le constat de certains chercheurs montre ainsi la difficulté de dépasser certains présupposés occidentaux. Afin de justifier l'emploi du terme «villes en développement» par exemple, M. Bertrand «convenons de la formule pour couvrir la variété des Suds [sic], dont les différences de taux, de rythme et de concentration de l'urbanisation sont connues, et même si les références théoriques sur la ville restent de fait largement empruntées aux trajectoires historiques du Nord» (Bertrand, 2010: 8). Les recherches plus théoriques visant à remettre en cause la portée générale de notions forgées en Occident, comme la ségrégation, la citadinité et l'urbanité, ou encore le développement durable, restent finalement rares.

Pourtant, certains auteurs revendiquent justement de nouvelles manières de concevoir les dynamiques urbaines dans les villes non occidentales. Chenal, Pedrazzini et Kaufmann, à propos de la publication récente d'un ouvrage, écrivent:

une théorie de la ville africaine est donc nécessaire, pour autant qu’elle soit également une théorie africaine de la ville. Sans quoi nous nous condamnons à répéter les erreurs parfois dramatiques commises par les urbanistes et surtout à répéter les solutions mises en place pour répondre de manière erronée à ces erreurs. [...] Une alter-modernité qui n’est plus le fruit de métissages entre un modèle blanc et colonial et des pratiques noires et indigènes, mais qui résulte d'un autre «chemin», vernaculaire peut-être, définissant ses propres règles, affirmant avec résolution sa modernité autre (2009a).

Si les auteurs annoncent la nécessité d'une planification des villes africaines métissées, postcolonialistes et postcapitalistes, on précise peu les modalités concrètes de la mise en œuvre d'un tel projet. Par ailleurs, leur conception de la ville africaine semble tributaire de notions rattachées à un certain culturalisme réifiant la notion de ville africaine, comme celles de modernité, de progrès et de développement, à l'inverse de ce que revendiquent justement les postures postcoloniales. On peut alors formuler deux questions, plus larges et transversales. Tout d'abord, l'annonce d'une indispensable approche autochtone de la planification urbaine dans les villes non occidentales 
peut-elle se soustraire à une remise en cause préalable des cadres conceptuels normatifs qui œuvrent à la caractérisation de ces villes? Ensuite, le dépassement du positivisme et de la rationalité occidentale doit-il servir un relativisme permettant d'échapper à toute tentative d'élaboration de nouveaux cadres conceptuels précis qui, tout en analysant ses biais et en assumant sa perpétuelle incomplétude, permettrait d'alimenter le débat et de faire évoluer les pratiques?

L'évolution des recherches francophones témoigne donc d'une diversification des sujets et des objets de recherche, d'une transversalité thématique et scalaire plus importante, d'une ouverture plus manifeste aux villes situées en dehors du monde occidental, ainsi que d'un renouvellement théorique et épistémologique, certes diffus et parcellaire, mais bien réel. Nous retrouvons de telles tendances au sein des recherches anglophones en général. Ces dernières illustrent là encore un repositionnement commun des recherches urbaines sur les villes non occidentales.

\section{Le renouvellement des recherches anglophones}

Défendus plus explicitement par leurs auteurs, les grands courants épistémologiques structurant l'évolution de la recherche anglophone portant sur les villes du Sud, depuis les années 1990, sont plus facilement repérables que ceux de la recherche francophone. La profusion de nouveaux concepts et méthodes donne l'impression d'un renouveau plus important du champ de recherche ${ }^{2}$. Pourtant, une étude plus approfondie de leurs contenus montre qu'elles procèdent bien souvent de constats similaires, même si les fonds théoriques et les inscriptions disciplinaires peuvent être très différents.

2. De manière évidente, l'importance de la production scientifique anglophone et l'internationalisation de la langue anglaise expliquent structurellement l'influence de cette production dans la recherche urbaine. 


\section{Une remise en cause des approches classiques}

Les approches critiques, s'inspirant ou se revendiquant explicitement des courants néomarxistes ou postmarxistes, postcolonialistes et postdéveloppementalistes ont largement été attribuées au renouvellement des études urbaines anglophones depuis les années 2000. Plus généralement, le tournant culturel des années 1960, le postmodernisme depuis les années 1970 et le tournant spatial à partir des années 1980 (Soja, 1980) ont contribué à la remise en cause des cadres traditionnels de l'urbanisme, de la rationalité occidentale et de la place des villes non occidentales dans les études urbaines. En conséquence, l'évolution des approches théoriques et empiriques du monde universitaire anglo-américain doit certainement beaucoup au renouvellement du champ de l'urbanisme des années 1970-1980.

En effet, au cours des années 1980, la position et l'action des urbanistes - en ce qu'elle implique une position de pouvoir vis-à-vis de la sphère publique - font l'objet de vives critiques. Pour bon nombre d'auteurs, il devient évident que derrière la rationalité et l'objectivité présupposées des urbanistes se dessinent des savoirs et des techniques socialement, historiquement et idéologiquement situés. Dans les années 1970-1980, le renouvellement très intense des théories en urbanisme et aménagement ont ainsi abouti à l'émergence de nouvelles manières de concevoir l'action et ses fondements réflexifs. On pense par exemple au tournant communicationnel, ou encore à l'influence du postmodernisme dans l'émergence de nouvelles utopies urbaines où justice sociale et citoyenneté trouvent une place centrale. Nous pouvons aussi mentionner les contributions de l'urbanisme radical, largement opposé à la planification rationnelle et institutionnelle, prônant des approches par le bas, collectives et collaboratives, pour la mise en œuvre de villes plus justes.

Les apports de la pensée critique en urbanisme et en études urbaines ont permis de constituer un nouveau répertoire critique de la pensée urbaine anglophone, sur lequel se sont appuyés de nombreux chercheurs travaillant sur les villes non occidentales. Il s'agit ainsi de 
repenser les pratiques et les approches urbanistiques en considérant les rapports de pouvoir et les registres d'action qu'elles supposent, tout en localisant davantage la pensée urbaine, tant dans l'histoire que dans l'espace. Au cours des années 1980-1990, les objets, les approches et les échelles d'analyse des études urbaines évoluent rapidement. L'importance donnée au local, la prise en compte des groupes sociaux subalternes, les approches socioculturelles ou encore les études portant sur les marges urbaines ont largement influencé les recherches portant sur les villes non occidentales. Mais surtout, le renouveau des études urbaines anglophones dans ce qu'on nomme souvent le «Sud global» semble particulièrement lié, comme dans la recherche francophone, à la globalisation du fait urbain.

Dès les années 1980-1990, mais plus particulièrement au cours des années 2000, un nombre croissant de chercheurs critiquent la faible représentativité des villes non occidentales dans les études urbaines anglophones. On pose ce débat notamment en réponse au succès des théories portant sur les grandes dynamiques métropolitaines globales, ou encore en réaction aux recherches traitant de phénomènes urbains considérés comme universels, tels que la périurbanisation. Selon de nombreux auteurs, en proposant des approches typologiques et une lecture hiérarchique des logiques de métropolisation, les théories sur les villes globales ont accentué la vision d'une globalité urbaine réductrice, dominée par quelques grandes métropoles et où les espaces secondaires ou périphériques ne chercheraient qu'à imiter les grandes métropoles mondiales. Ainsi une trop grande attention portée aux métropoles des pays émergents a-t-elle proportionnellement caché les dynamiques urbaines de villes situées censément à leur périphérie.

$\mathrm{Au}$ cours des années 2000 donc, de nombreux chercheurs du monde universitaire anglophone ou anglo-américain se positionnent en faveur d'une diversification des recherches urbaines vers les villes situées en marge des grandes logiques de métropolisation. Robinson (2006), tout en soulignant l'apport important des études urbaines globales, a justement montré comment cette hiérarchisation procède 
de visions occidentalo-centrées et positivistes, qui ont tendance à interpréter le phénomène urbain par le seul prisme de la trajectoire historique des villes occidentales (industrialisation, périurbanisation, mises en réseau, etc.). Dans ce contexte, la force du diptyque modernité/développement freinerait la compréhension des dynamiques urbaines locales, toujours prises entre processus endogènes et exogènes. Finalement, il ne faudrait plus les considérer comme une exception à l'ordinaire urbanistique dont le cœur se trouverait en Occident. L'idée de ville ordinaire proposée par Robinson et appliquée aux villes non occidentales, bien que plus ancienne (Amin et Grahama, 1997), viserait ainsi à dépasser l'exotisme qui ponctue bon nombre de travaux portant sur les villes des pays en développement, dont le caractère extraordinaire renvoie bien souvent à l'anormalité présupposée de leur évolution, si souvent décriée depuis les années 1960.

L'attention qu'on porte aux villes périphériques devient alors un parti pris épistémologique. Pour Ananya Roy, il faut se garder de confondre la globalité du fait urbain avec l'universalité des dynamiques urbaines (2015). Pour ce faire, il devient indispensable de proposer de nouveaux cadres conceptuels produits à partir d'une déconstruction des concepts normatifs élaborés en Occident. Ce positionnement impliquerait alors de provincialiser les théories urbaines dominantes et d'appliquer ce principe de décentrement aux recherches urbaines dans le Sud global. Nous verrons plus loin les critiques que nous pouvons porter à de tels arguments.

Une divergence croissante apparaît ainsi entre les approches d'inspiration marxistes d'un côté, et les recherches critiques imprégnées du tournant culturel et du postcolonialisme de l'autre. Pour ces dernières, les postures critiques d'inspiration marxiste, portant par exemple sur la gentrification, le néolibéralisme, l'habitat informel, les logiques de rente ou encore les inégalités sociospatiales, tendent justement à sous-estimer la complexité du local, voire à transposer implicitement une approche élitiste de la théorisation en sciences sociales à propos des villes non occidentales. Ces débats témoignent de l'évolution des fondements des recherches critiques, mais aussi de 
la recherche de solutions théoriques visant à dépasser les approches centre-périphérie, celles sur le développement et la modernité, ou encore celles portant sur les rapports de classes.

\section{La consolidation de nouveaux champs théoriques et thématiques}

Malgré ces divergences, les recherches critiques partagent la volonté de mettre en avant les populations, les groupes sociaux, les espaces et les moments historiques que le passé colonial a contribué à garder sous silence. L'appel au renouvellement de la recherche (théorique, thématique, méthodologique, etc.) favorise alors l'émergence de nombreux concepts rendant compte des pratiques urbanistiques du quotidien, du bas ou contestataires. Ce faisant, on propose des solutions de rechange aux logiques de planification et d'aménagement imposées d'en haut, souvent inspirés de modèles transnationaux issus de la production urbaine capitalistique globalisée.

Comme pour les recherches urbaines francophones, le tournant global des sciences sociales, puis l'attrait renouvelé envers le local et les approches ethnographiques, ont largement porté l'évolution récente du champ des études urbaines anglophones. Cependant, les recherches anglophones se sont aussi attachées à redéfinir le global par le local, dans l'optique d'une reconsidération de ce qui est communément désigné comme centre ou périphérie. En ce sens, les grandes métropoles comme les plus petits centres urbains doivent faire l'objet d'approches ethnographiques rendant compte de leurs singularités tout autant que de leurs potentiels de globalité. Il s'agit par ailleurs de repenser l'engagement critique des chercheurs à l'heure de l'urbain généralisé, notamment à propos du droit à la ville.

Parallèlement et encore à l'instar des recherches urbaines francophones, de nouvelles échelles d'analyse des dynamiques urbaines voient le jour. On notera, par exemple, une attention de plus en plus manifeste pour les approches comparatives, d'où émergent de nouvelles propositions théoriques largement inspirées des approches relationnelles en géographie et en études urbaines, des théories transnationales, ou 
encore du mobility turn (Urry, 2007). En conséquence, les comparaisons Sud-Sud se popularisent, et avec elles l'étude des dynamiques de circulation et de transfert de modèles urbains, de politiques publiques, de pratiques et de théories urbanistiques, ou encore de modes de vie. Pour les chercheurs qui se situent au croisement de la géographie et de l'urbanisme, il devient nécessaire de réfléchir aux nouvelles échelles que suppose la globalisation du phénomène urbain. Par exemple, l'échelle translocale permettrait de mieux analyser les différentes formes de tensions, entre les circulations d'influences urbaines à l'échelle globale d'une part et leurs logiques d'ancrage et de territorialisation à l'échelle locale d'autre part.

Ces contributions théoriques s'appuient par ailleurs sur de nouvelles complémentarités entre l'interdisciplinarité, le décentrement des positionnements théoriques et la diversification des objets de recherche. Malgré cette volonté de renouvellement, de nombreux auteurs puisent dans un registre théorique forgé en Occident, à partir de recherches portant sur les sociétés occidentales. On peut par exemple souligner la forte influence de la French Theory qui, portée par sa popularité dans les recherches critiques et les cultural studies anglo-saxonnes, vient nourrir une remise à niveau du cadre conceptuel des recherches urbaines sur les villes non occidentales. La mobilisation accrue de concepts comme les assemblages, la gouvernementalité, ou encore la théorie de l'acteur-réseau en sont quelques exemples.

Cette diversification théorique s'accompagne d'une évolution des approches thématiques, qui illustrent une remise en question des approches urbanistiques au sein des villes non occidentales. La critique des approches considérées comme traditionnelles de l'urbanisme (rationnelles, holistes, institutionnalisées, etc.) se traduit par une remise en cause des processus hégémoniques sous-jacents à la production urbaine contemporaine, comme le néolibéralisme, le capitalisme ou la privatisation de la fabrique urbaine, ainsi que des logiques politico-économiques qui les portent. Une partie des approches radicales devient particulièrement attentive aux initiatives urbanistiques locales, ainsi qu'à la manière dont les populations 
subalternes s'opposent aux modes de production dominants et proposent des solutions. On s'intéresse par exemple aux stratégies d'autoorganisation, de contestation, d'opposition et de lutte des habitants pour mener à bien différents projets communautaires à l'échelle locale, que ce soit en contexte autoritaire ou au sein de régimes économiques plus libéraux. D’autres chercheurs montrent ainsi comment l'urbanisme devient un acte militant et politique, ou encore citoyen.

Cette courte présentation de l'évolution récente du registre thématique et conceptuel des études urbaines anglophones (appartenant majoritairement au monde universitaire anglo-américain) sur les villes non occidentales ne peut prétendre à l'exhaustivité. On aurait pu évoquer bien d'autres thèmes au cours des pages qui précèdent. Nous pouvons penser, par exemple, à l'importance croissante des études sur le genre, la jeunesse, les nouvelles problématiques environnementales, ou encore sur les notions d'identité, de citoyenneté et d'espace public. De même, nous avons dû laisser de côté des champs thématiques importants, comme l'économie urbaine ou la planification régionale. Cependant, la mise en perspective de la littérature francophone avec l'anglophone permet d'émettre quelques constats intermédiaires et de formuler des propositions allant dans le sens d'une meilleure identification des registres théoriques et méthodologiques des recherches urbaines sur les villes non occidentales.

\section{Pour un champ de recherche sur les villes non occidentales}

Comme nous l'avons évoqué, si les recherches urbaines francophones sur les villes non occidentales sont riches et se sont largement diversifiées depuis les années 1980, la transition de «villes du Tiers-Monde» à «villes du Sud» s'est finalement peu accompagnée de travaux réflexifs portant sur l'évolution récente des cadres théoriques et méthodologiques. On peut s'interroger sur les raisons de ce manque et sur les manières d'y remédier. 


\section{La recherche francophone face à l'altérité urbaine}

En comparaison avec les travaux anglophones, les recherches urbaines francophones sur les villes non occidentales semblent s'inscrire beaucoup moins dans un champ de recherche spécifique et revendiqué. Cette situation renvoie à des différences de positionnement scientifique vis-à-vis du Sud global, à la place de la théorie dans les recherches urbaines et à l'articulation des différentes disciplines universitaires traitant des enjeux urbains.

Dans les recherches angloaméricaines, la remise en question des approches traditionnelles de l'urbanisme, le foisonnement des recherches critiques et le décentrement tant géographique que conceptuel des études urbaines sur les villes non occidentales se sont paradoxalement appuyés sur une mise en exergue particulièrement manifeste de la partition Nord-Sud. La ligne de partage ne s'est donc pas estompée, elle s'est plutôt renforcée à la faveur de la place plus grande qu'ont prise les Global South Studies dans le monde anglosaxon depuis les années 1990. De nombreux auteurs s'en défendent cependant. Pour Sophie Watson (2014), nommer explicitement les villes du Sud ou les villes non occidentales permet d'insister sur la diversité des dynamiques urbaines à l'heure de la globalisation urbanistique, tout en remettant en question l'imposition de la rationalité occidentale dans l'urbanisme et l'aménagement.

Les recherches urbaines francophones, quant à elles, semblent se tenir à distance de ces débats et y font peu face de manière explicite. On peut invoquer ici différentes raisons. La difficile identification d'un répertoire théorique dans les recherches urbaines en général freine la constitution de champs de recherche spécifiques, comme pourrait l'être celui portant sur les villes non occidentales. Comme l'a déjà souligné le collectif Champ libre ${ }^{3}$ ?, le cadre théorique des recherches

3. Le collectif Champ libre?, constitué récemment, rassemble de jeunes enseignantschercheurs français en aménagement et urbanisme. Le collectif s'est formé dans le cadre de l'organisation d'une conférence en janvier 2016 intitulée "Champ libre? L'urbanisme et l'aménagement à l'épreuve des cadres théoriques». L'appel à communication de l'événement partait notamment du constat de la difficulté à identifier les cadres théoriques de l’aménagement et de l’urbanisme en France. 
urbaines dans le monde francophone (principalement à propos des villes du Nord) s'inscrit dans un certain «flou» épistémologique, que la quasi-absence d'un fonds théorique et disciplinaire commun a favorisé. Comme le précise le collectif, la tension entre recherches théoriques et pratiques en urbanisme, le manque d'enseignements spécifiques, ou encore l'éclatement du champ entre différentes disciplines ne facilitent pas la mise en valeur d'un fonds théorique et conceptuel pourtant important. Comme le remarque Sherrer (2010), il est effectivement plus difficile de participer à des débats appartenant à un champ de recherche articulé autour d'un objet - la ville - lorsque l'on inscrit principalement sa pensée dans une discipline, comme c'est le cas en France et, plus largement, dans le monde francophone. À la suite de ce que nous avons pu montrer, le dispersement de la théorie au sein d'approches principalement thématiques et régionales sur les villes non occidentales renvoie donc plus largement à une réalité que partagent les recherches urbaines en général.

Ces logiques disciplinaires internes sont aussi tributaires de la dévolution à la géographie des réflexions théoriques portant sur le Sud (en tant que catégorie, objet ou espace de recherche). Cette réalité a sûrement accentué la frilosité des recherches urbaines à se saisir des enjeux théoriques et épistémologiques soulevés par cette catégorisation. Pour beaucoup de chercheurs ou de praticiens de l'urbain, isoler la ville non occidentale de la ville en général présente le danger de tomber dans la «trappe théorique» de la dichotomie Nord-Sud et de la virulence des débats à cet endroit, dont les géographes restent les principaux animateurs.

Pour ces derniers, le Sud constitue bien évidemment une catégorie ou un objet à déconstruire et à dépasser. Les chercheurs se sont ainsi attachés à dénoncer la séparation subjective Nord-Sud et à remettre en question son tropicalisme latent, sujet de débat par ailleurs ancien ${ }^{4}$. La simple mention du terme «Sud», «Tiers-Monde» ou «non occidental» participe de la perpétuation d'une partition civilisationnelle

4. Voir notamment le numéro 4 de L'Espace géographique intitulé «Géographie tropicale - géographie du Tiers Monde» publié en 1984. 
ou culturelle du monde, car «nommer, c'est créer un peu» (Raison, 2007). Il devient alors indispensable de se «délivrer de l'exotisme» (Hancock, 2007) et de « désoccidentaliser la pensée urbaine» (Choplin, 2012). Dans ce contexte, défendre la constitution d'un champ de recherche spécifiquement attaché aux villes non occidentales s'avère difficile. Pourtant, ces dynamiques disciplinaires et ces postures présentent certaines limites qui peuvent nuire, c'est du moins l'argument ici, aux recherches portant sur les villes non occidentales.

\section{Au cœur du renouvellement des recherches urbaines}

Si la plupart des chercheurs sont aujourd'hui conscients du caractère ethnocentré du découpage Nord-Sud, celui-ci n'en reste pas moins un vecteur puissant de l'organisation institutionnelle et scientifique de la recherche urbaine. Comme nous l'avons mentionné, la domination des institutions occidentales dans la production scientifique mondiale, la lecture des dynamiques urbaines globales à partir de concepts forgés au Nord, ou encore la force du rationalisme occidental dans la considération des dynamiques urbaines mondiales en sont quelques exemples.

Ainsi, l'altérité et l'exotisme ne cessent pas d'exister dès lors que l'on en fait la critique ou qu'on les passe sous silence. Comme nous l'avons vu précédemment, dépasser la dichotomie Nord-Sud n'empêche en rien le maintien de cadres théoriques et méthodologiques normatifs (universalisme, développement, modernité, etc.). Un décalage se fait alors sentir entre une remise en cause théorique de cette partition du monde et le maintien de ces représentations dans les logiques institutionnelles et scientifiques structurant la production des savoirs. Pourtant, il existe une différence entre l'indispensable effort de toujours remettre en cause nos représentations socialement situées (qui s'attachent à des constructions culturelles et sociales jamais universelles) et la reconnaissance de l'effet de ces représentations sur la recherche scientifique et les pratiques professionnelles. Par exemple, s'il s'agit de désoccidentaliser la pensée urbaine, il faut 
identifier la substance de cette occidentalisation et non la taire, au risque de favoriser le maintien de représentations dichotomiques et simplistes de la réalité urbaine globale. Considérer le principe ontologique de celui qui parle, recherche ou agit, ainsi que les cadres conceptuels et méthodologiques qui structurent son action, permet de produire une réflexivité épistémologique nécessaire à l'évolution des recherches urbaines. En d'autres termes, si la géographie examine son rôle dans la production d'une "assignation à territorialité " (Hancock, 2008: 117), la recherche urbaine doit elle aussi s'intéresser de manière plus systématique aux différentes formes d'assignations à urbanité qu'elle produit.

Dans ce contexte, si isoler les villes non occidentales comme champ ou objet d'analyse représente certes une catégorisation faussée et une essentialisation trompeuse, cela permet en revanche de mieux défendre une diversification de la recherche urbaine francophone et une plus grande représentativité des villes non occidentales dans la production des savoirs. Par ailleurs, cette classification facilite l'identification de discours et de représentations dominants, étape préalable indispensable à leur déconstruction. En ce sens, faire exister la ville non occidentale, de manière temporaire et à des fins épistémologiques, doit donc aussi s'accompagner d'une indispensable critique de cette catégorisation.

Prendre à bras-le-corps la thématique des villes non occidentales permettrait la constitution d'un champ de recherche plus balisé et structuré par des écoles de pensée et des positionnements théoriques, voire politiques, plus assumés. La constitution d'un tel champ permettrait de confronter plus systématiquement différentes approches et théories, et de maintenir ainsi un espace de débat constructif favorisant une plus grande transversalité des approches thématiques. Il serait alors possible de rassembler les théories et les concepts des recherches urbaines sur les villes non occidentales. Cela pourrait passer par la production de manuels, d'événements scientifiques spécifiques, de cours universitaires dédiés, ou encore par la création de groupes ou de collectifs de recherche. 
Un tel positionnement n'empêche en rien les approches valorisant les recherches thématiques dépassant les catégories Nord-Sud et ne contredit pas non plus les travaux insistant sur les approches relationnelles de la fabrique urbaine (globalisation urbanistique, circulation de modèles, notamment Sud-Sud, etc.). Une plus grande mise en valeur des registres théoriques et des méthodes mobilisés pour l'étude des villes non occidentales augmenterait au contraire la capacité des chercheurs et des praticiens à identifier les modalités de circulation de concepts, ainsi qu'à valoriser les méthodes et les théories produites par les institutions et les chercheurs non occidentaux, qui devraient par ailleurs occuper une position beaucoup plus centrale dans les recherches urbaines en général. Un bornage plus précis de ce champ de recherche permettrait de favoriser les échanges, ainsi que la circulation de concepts et de méthodes, entre chercheurs occidentaux et non occidentaux. En d'autres mots, une recherche urbaine attentive à la notion d'altérité et à sa capacité normative, induite par les rapports de production inégaux des savoirs et de l'expertise technique, permettrait d'assurer un renouvellement pérenne et une diversification de la recherche urbaine francophone, ainsi que des pratiques en aménagement et urbanisme. Les différentes parties de l'ouvrage s'insèrent justement dans cette volonté.

\section{Présentation de l'ouvrage}

$\mathrm{Au}$ fil du travail collectif impliquant les auteurs de l'ouvrage, trois grands thèmes de réflexion transversaux ont rapidement émergé: les enjeux des espaces publics dans le devenir des villes non occidentales; l'importance des jeux d'échelles dans la recherche et la pratique; les terrains et les méthodes de la recherche urbaine sur les villes non occidentales. Ces thèmes représentent les trois grandes parties de l'ouvrage. 


\section{L'espace public, élément constitutif et constituant de la ville}

La première partie de l'ouvrage concentre son regard sur un thème majeur des recherches urbaines: les espaces publics, qui occupent une place de plus en plus centrale au sein des travaux portant sur les villes non occidentales. Si le point de départ représente bien la notion d'espace public au sens d'espace de débat politique, les études urbaines $\mathrm{y}$ associent la notion d'espaces publics (au pluriel), c'est-à-dire les espaces et les lieux où s'élabore la relation entre les citadins et la sphère publique du débat politique ou citoyen. Les espaces publics traversent en ce sens de multiples thèmes de recherche en même temps qu'ils posent un grand nombre de questions, dont une majeure: comment se construit concrètement la citadinité aujourd'hui? Cette interrogation s'avère d'autant plus cruciale au regard de la privatisation croissante de l'aménagement des villes, du morcellement accru des territoires urbains, de la disparition ou de la recomposition des lieux qui faisaient précédemment office d'espaces publics, ou encore de l'émergence de nouvelles formes de coercition et de contrôle des citadins. Cependant, les espaces publics se situent bien souvent là où on ne les attend pas et les acteurs urbains s'accommodent régulièrement des transformations contemporaines de la ville. L'espace public n'a donc pas d'existence propre, c'est-à-dire qu'il n'existe pas indépendamment de l'acteur urbain et de la structure sociopolitique qu'il produit, et dont il dépend.

Aniss M. Mezoued nous rappelle en ce sens que l'espace public ne va pas de soi : il est le produit de «récits » et se fait l'écho de stratégies d'acteurs. Les espaces publics s'avèrent alors stratégiques, car ils se situent à l'interface de différentes manières de percevoir la ville, de la produire et de l'aménager. À l'heure où l'expression «fragmentation urbaine» fait bien souvent figure de tautologie, les espaces publics représentent le substrat collectif à la fois physique et idéel nécessaire au dépassement des intérêts particuliers. Cependant, il ne suffit pas de désigner certains lieux comme espaces publics pour les faire exister. Ces derniers ne peuvent en effet représenter une simple 
solution aux espaces privés, mais peuvent devenir des espaces de médiation entre les sphères publique et privée de la ville.

Pauline Bosredon associe ainsi les thèmes du patrimoine et des espaces publics au sein d'une relation multidimensionnelle: dans quelle mesure le patrimoine représente-t-il un espace public, et comment certains espaces publics sont-ils patrimonialisés? Voici une mise en perspective particulièrement féconde, tant le patrimoine, tout comme l'espace public, représentent des notions à la fois discursives et concrètes, représentées et appropriées, idéologiques et d'une banale quotidienneté. L'auteure, en comparant des cas d'étude au Brésil et au sein des Territoires palestiniens occupés, nous explique comment le processus de patrimonialisation peut être le support de revendications sociales et culturelles fortes. Dans le cas du Brésil, elles peuvent aboutir à un ancrage plus marqué du caractère public d'espaces urbains centraux. Au sein des Territoires palestiniens occupés, les discours et les stratégies d'acteurs font écho au conflit militaire larvé. Les espaces patrimoniaux, à la fois publics (la vieille ville) et privés (les logements), participent de la construction d'un espace public, qui se meut en véritable arène où s'affrontent les belligérants des conflits politiques et idéologiques. Le rôle médiateur de l'espace public est donc à remettre en question, en même temps que la neutralité du processus de patrimonialisation qu'on admet parfois; comme nous le précise l'auteure, et en écho à Aniss Mezoued, la relation entre espaces publics et patrimoine au Brésil et au sein des Territoires palestiniens occupés se comprend moins dans les faits que dans les récits.

Aux dimensions idéelles et politiques des espaces publics s'ajoute une dimension tout à fait physique, qui renvoie notamment à l'aménagement d'espaces ouverts aux habitants au sein de quartiers urbains. À ce titre, Cécilia Montoya Antich évoque le rôle de la participation citoyenne dans la création d'espaces publics à Lima, capitale du Pérou. S'il est aujourd'hui à la mode de promouvoir un aménagement des espaces urbains par les habitants, il apparaît indispensable de remettre en question les supposés bienfaits des politiques de décentralisation 
municipale et de la participation du citoyen-citadin dans la construction de la ville. En effet, l'auteure nous montre bien que la généralisation de budgets participatifs à Lima et le désengagement des autorités municipales participent d'une fragmentation des espaces urbains et d'une redéfinition des fondements mêmes des espaces publics (qui devraient être, en théorie, des espaces ouverts à tous). En conséquence, si les budgets participatifs devaient permettre aux quartiers les plus précaires de pallier leur manque de moyens financiers et techniques, leur généralisation favorise de grandes disparités d'aménagement entre les différents quartiers de la ville, qui se font le reflet des inégalités sociospatiales à l'échelle de l'agglomération.

Une plus grande participation citoyenne ne mène donc pas automatiquement à une amélioration du caractère public des espaces urbains, même dans le cas où les espaces publics semblent se multiplier. À l'inverse, certains lieux, qu’on ne considère généralement pas comme des espaces publics, pourraient tout à fait assumer ce rôle; en évoquant l'école comme un espace public à reconsidérer, Valeria Cartes Leal s'attaque justement à cette problématique. Premier lieu de l'élaboration du rapport entre l'homme et la ville comme espace de citoyenneté, l'école est rarement considérée comme un espace public local à partir duquel s'élaboreraient de nouvelles formes de citadinité. Car, comme nous le dit l'auteure, l'école n'est pas seulement un lieu où l'on apprend théoriquement à être citoyen; c'est aussi un espace vécu par lequel se construit notre rapport au quartier, à la proximité et à la vie en commun. À ce titre, l'auteure propose la notion d'« urbanisme scolaire», qui prendrait en considération l'articulation de l'école aux espaces publics de la ville tout en redonnant à l'école son potentiel d'urbanité, lequel s'exprime bien souvent lors de crises sociales, comme ce fut le cas Chili. À la fois vecteur et incubateur des transformations sociopolitiques de la cité, l'école cesserait ainsi d'être cantonnée à un fonctionnalisme éducatif pour véritablement prendre place dans l'espace public urbain qui lui est dû. 


\section{Les jeux d'échelles au cœur du phénomène urbain}

Les jeux d'échelles conditionnent et structurent tant les positionnements scientifiques que les objets et les thèmes de recherche. Il ne s'agit cependant pas de se demander à quelle échelle on doit appréhender la ville, car la réponse serait immanquablement «à toutes »; il apparaît plutôt nécessaire de saisir, d'une part, pourquoi toutes les échelles sont nécessaires à la compréhension du phénomène urbain et, d'autre part, ce que chaque échelle d'analyse soulève comme enjeux et questions, tant pour la recherche que pour la pratique. Au cours des années 1990, un important débat, encore présent aujourd'hui, s'animait autour de la question de l'uniformisation des espaces urbains mondiaux et des processus de métropolisation sous l'effet de la globalisation économique, sociale et culturelle. Ce débat a notamment privilégié des échelles d'analyse très petites, à l'échelle du monde, des continents, des régions et des grandes aires urbaines. $\mathrm{Si}$ ces discussions semblent aujourd'hui quelque peu datées, tant bon nombre d'auteurs s'accordent à considérer la ville comme le produit d'un métissage entre des réalités à la fois globales et locales, la question des échelles de la ville reste tout à fait féconde, comme en témoigne l'importance croissante qu'on accorde à l'intermédiarité, au transscalaire ou au multiscalaire. La question des échelles de la ville s'inscrit actuellement au sein de thèmes de recherche à la fois plus larges et plus précis, qui renvoient notamment à la généralisation de certains sujets (l'habiter, les pratiques habitantes, les stratégies et les discours d'acteurs, etc.) et objets (le quartier, la rue, les marchés, etc.) de recherche, qui s'appréhendent tant aux petites échelles qu'aux grandes. La deuxième partie de cet ouvrage s'inscrit résolument dans cette dynamique, tout en proposant une double perspective: elle examine non seulement les échelles de la fabrication urbaine, mais aussi la fabrication des échelles de la ville.

Mauricio Anaya présente en ce sens un regard tout à fait original sur une ville secondaire bolivienne, Cochabamba, en montrant comment la réflexivité peut et doit entrer dans les manières dont nous 
pensons et réalisons la fabrication urbaine aujourd'hui. En prenant le parti de considérer que théorisation et pratique sont indissociables en aménagement et urbanisme, l'auteur oppose fragmentation urbaine et urbanité, en montrant comment espaces urbains, gouvernance et pratiques habitantes se font écho au sein d'une relation systémique trop souvent sous-estimée. La question de l'échelle de la ville est par ailleurs posée au sujet du caractère "moyen» de la ville de Cochabamba, que l'auteur qualifie de «ville comme toutes les autres», à l'instar de la «ville ordinaire» de Robinson (2006). On privilégie donc doublement la grande échelle: en considérant une petite ville comme toutes les autres petites villes, de surcroît à l'échelle locale. Le «régime d'urbanité» dont nous parle l'auteur transcende ainsi les échelles d'analyse, pour représenter un outil très concret permettant de repenser les sphères publique et privée de la cité, ou encore de relever ce qui fait la continuité par-delà l'apparent morcellement de l'espace urbain.

Issa Sory étend le questionnement à la gouvernance de la ville en opposant la planification stratégique au droit à la ville, thème de recherche qui s'est généralisé au sein de la littérature francophone en études urbaines au cours des années 2000, notamment à partir d'études de cas en Afrique du Sud et en Amérique latine. Parfois expression valide, parfois thème central des études urbaines critiques renouvelées, le droit à la ville n'est pas seulement une proposition théorique voulant souligner les desseins sociopolitiques derrière les processus de marginalisation; il représente aujourd'hui un véritable cadre théorique permettant de nouvelles analyses quant aux processus sociaux, spatiaux, économiques et politiques menant à la ségrégation et à la stigmatisation de certaines populations. En couplant le droit à la ville à la planification stratégique, Issa Sory met finalement en opposition deux échelles d'analyse. L’une, globale, renvoie au passage du rationalisme au stratégisme dans les manières d'envisager l'encadrement du développement urbain; l'autre, locale, montre comment la planification stratégique fait écho à la nature même du régime politique burkinabè, qui joue justement avec les échelles de la ville 
pour mieux asseoir ses stratégies, notamment en s'accommodant d'une participation citoyenne qui prend l'aspect d'un simulacre démocratique.

En proposant le «chemin» comme objet de recherche, Bianca De Marchi Moyano s'attaque à l'essence même de la multiscalarité: le réseau. Là encore, les multiples échelles d'analyse ne sont pas seulement spatiales: elles sont aussi politiques et temporelles. En effet, le chemin renvoie à la fois aux échelles de la mobilité, à l'articulation des politiques territoriales dans le contexte d'un État plurinational en formation, ainsi qu'à la concordance de temporalités qui rythment différentes formes de territorialité. Dans une volonté de considérer le chemin de manière transversale, l'auteure propose une posture originale: le chemin doit être un facteur d'ancrage permettant de dépasser les tensions liées à l'aménagement de l'espace et à la gestion des flux. Pour cela, des propositions très concrètes sont formulées: considérer que l'aménagement des réseaux relève de dynamiques processuelles plutôt que politiques; appréhender le territoire dans sa porosité; mobiliser le paysage comme objet médiateur et échelle d'action dans la gestion de la mobilité et le développement des réseaux.

\section{Les chercheurs/praticiens en études urbaines et leurs terrains}

La dernière partie de l'ouvrage suggère des pistes de réflexion relatives au positionnement des chercheurs et des praticiens vis-à-vis de leurs terrains, leurs objets et leurs sujets de recherche. Ces questionnements, qui concernent peu ou prou l'ensemble des professionnels et des chercheurs s'appuyant sur des travaux empiriques, ne renvoient pas seulement aux outils et méthodes mobilisés: ils évoquent plus largement le rapport à l'altérité, la complexe articulation entre jeux d'échelles et d'acteurs, ainsi que l'intérêt d'adopter une posture réflexive dans l'élaboration et l'application d'outils d'aménagement et d'urbanisme très concrets. Le rapport entre les chercheurs en urbanisme et aménagement et leurs terrains de recherche reste un sujet marginal au sein des études urbaines francophones, alors même que 
la plupart des sciences sociales ont, parfois depuis longtemps, ouvert cette boîte noire. En conséquence, les chercheurs et les praticiens en recherches urbaines ont tendance à puiser dans d'autres disciplines les outils et les théories leur permettant de mettre en perspective leurs recherches et leurs actions. Une question plus large se dessine alors: quelles sont les particularités des approches empiriques des recherches urbaines et du positionnement des chercheurs et des praticiens visà-vis de celles-ci ? Si la dernière partie de cet ouvrage ne s'attaque pas de front à cette question, les interrogations des auteurs permettent d'ores et déjà de souligner différents enjeux propres aux recherches urbaines et aux pratiques de l'urbanisme et de l'aménagement.

Dans sa contribution, Claire Simonneau confronte la normativité développementaliste à la réflexivité postcoloniale. La question de la normativité au sein des recherches urbaines revêt une importance cruciale, le rationalisme technique et technologique étant un paradigme particulièrement puissant chez les professionnels de l'aménagement urbain. Dans bon nombre de villes où l'aménagement et la gestion des espaces urbains souffrent du manque de moyens tant techniques que financiers, la normalisation des modes de gestion du développement urbain est d'autant plus importante qu'elle s'appuie souvent sur l'imposition d'outils et d'approches allochtones, généralement inadaptés à la réalité concrète des pratiques et des habitudes locales. La critique postcoloniale n'apparaît alors pas seulement théorique: elle peut aider, selon l'auteure, à mieux considérer les villes pour ce qu'elles sont, non pour ce qu'elles devraient être, et à mieux considérer les pratiques et les représentations des acteurs urbains locaux.

Divya Leducq, en évoquant la pluralité de ses terrains de recherche et de ses thèmes d'analyse, défend l'idée de «bricolage épistémologique et méthodologique», qu'elle définit non pas comme un positionnement hasardeux qui chercherait à rendre cohérent un ensemble de pratiques et de questions très diverses, mais plutôt comme une réponse aux enjeux multiples que supposent l'aménagement des villes d'une part, la nécessaire inventivité dont il faut faire preuve pour 
imaginer, créer et tester des outils capables de répondre aux enjeux urbains d'autre part. Cette notion de bricolage, qui renvoie assurément aux travaux de Lévi-Strauss, nous pousse peut-être à reconsidérer le mythe selon lequel la ville pourrait faire l'objet d'un aménagement parfait par la mobilisation d'outils urbanistiques qui se voudraient «exacts». Nous voyons bien ici que l'urbanisme et l'aménagement se posent les mêmes questions que bien des sciences sociales: que fait-on des partis pris qui se glissent entre la méthode et la réalité du travail de terrain? Pour l'auteure donc, le bricolage procède aussi de l'honnêteté scientifique, car il renvoie aux modes d'acquisition des données, aux échanges interculturels, ou encore aux enjeux linguistiques au sein de terrains variés. Dans ce contexte, la notion de bricolage ne serait-elle pas une forme d'objectivation méthodologique et épistémologique?

La remise en question du positivisme transparaît particulièrement lorsque Léandre Guigma évoque la difficile articulation entre les rôles de chercheur et de praticien. Mais surtout, Léandre Guigma nous rappelle que l'altérité se rencontre au pas de sa porte: Burkinabè et habitant de Ouagadougou, l'auteur n'en fait pas moins face à un difficile positionnement vis-à-vis de son terrain et des habitants qu'il interroge. Fort de tactiques et de stratégies qu'il adapte à la situation, et en miroir de son positionnement à la fois de chercheur et d'expert, l'auteur souligne que le discours de celui qui enquête est coproduit avec le «cherché», renvoyant en ce sens aux questions ontologiques que soulèvent notamment les postures et les identités multiples des acteurs en présence. Derrière ces réflexions émerge très certainement le «patricien réflexif» décrit par Donald Schön, qui appelait à une réflexivité dans et pour l'action. Le terrain cesse alors d'être une frontière entre le chercheur et le cherché, pour devenir un espace de production d'une réalité tangible en perpétuelle évolution, qui s'appréhende autant par «l'acteur-je» que par le «jeu d'acteurs».

En s'intéressant aux jeux d'échelles, aux espaces publics et au terrain, cet ouvrage propose une transversalité thématique importante, qui concerne un large spectre des recherches urbaines, notam- 
ment à propos des villes non occidentales. Nous espérons que cette production scientifique contribuera à promouvoir la recherche sur les villes non occidentales, en montrant l'intérêt d'une diversification des terrains de recherche et de la mise en œuvre d'un travail réflexif sur les méthodes et les concepts. 

PREM IÈRE PARTIE

L’eSPACE PUBLIC COMME MÉdiAtion 



\section{CHAPITRE 1}

\section{L'espace public comme fabricant de liens}

Aniss M. Mezoued

La notion d'espace public prend depuis quelques années une place importante dans le débat sur la ville. Cette notion s'est progressivement éloignée de celle d'espace de débat politique, de confrontation des opinions et de démocratie, telle que décrite par Habermas (1978), pour désigner le plus souvent, et plus simplement, une série d'espaces accessibles au public. Ces espaces ne sont pas toujours publics au sens juridique du terme. Des espaces privés comme des centres commerciaux peuvent aujourd'hui avoir un caractère public, par leur accès sans restriction au plus grand nombre. Malgré cette évolution de sens, qui fait par ailleurs débat, les espaces publics restent, dans l'imaginaire collectif, associés aux rues, aux places, aux parcs, aux jardins, etc. Il s'agit, dans la majorité des cas, d'espaces ouverts situés entre les espaces privés. Ce «vide» est au cœur des enjeux urbains contemporains. En effet, alors que les villes semblent se fragmenter de plus en plus, tel que le démontre Navez-Bouchanine (2002), les espaces ouverts jouent un rôle crucial au sein des approches urbanistiques contemporaines. Ils apparaissent, comme le dit Bernardo Secchi, «comme des matériaux urbains fondamentaux» (2006: 128), qui pourraient assumer le rôle d'intermédiaires entre les différents fragments urbains. Assurant le 
lien et les connexions dans le bâti, ces espaces ouverts et particulièrement les espaces publics semblent être garants d'une forme de cohésion de la ville, d'une certaine qualité de l'environnement urbain, voire, comme le précise Salat (2011), de la qualité de la vie urbaine.

\section{L'évolution des espaces publics}

Avant de s'intéresser à la question de l'espace public comme fabricant de liens, puis d'examiner les espaces publics d'Alger et la nécessité de les mettre au cœur de processus de coproduction de la ville, revenons brièvement sur l'importance de ces enjeux au regard de l'évolution contemporaine de la fabrique urbaine. Si les villes ont évolué très différemment et à des rythmes variés, elles sont pratiquement toutes passées des configurations traditionnelles de la ville pédestre à des configurations de plus en plus complexes et non homogènes, faisant éclater les distances et les échelles.

Si nous considérons la ville comme le résultat de la superposition, de la cohabitation et de la confrontation de récits divers, pris au sens de synthèses de l'hétérogène, il est alors possible de décrire son évolution non pas de manière chronologique, mais en fonction de ce que nous pouvons nommer des récits urbanistiques. Ces derniers varient selon les villes et des contextes, mais ont majoritairement mis en avant, depuis la moitié du $\mathrm{xx}^{\mathrm{e}}$ siècle, des logiques d'occupation foncière, d'infrastructures de transport, de production quantitative de logements, de zonage et de spécialisation des activités, de réorganisation du rapport centre-périphérie, etc. L'ensemble de ces actions, que le paradigme du progrès guide dans les pays dits du Nord et que celui du développement traduit dans les pays dits du Sud, a contribué à la fragmentation des villes et des territoires ${ }^{1}$. Chaque récit met en scène des acteurs ou des types d'acteurs territoriaux, des logiques de production urbaine et des espaces et des formes d'action sur la ville. Nos villes contemporaines seraient avant tout le résultat des actions spa-

1. Voir à ce propos les travaux de Magnaghi, 2003; Navez-Bouchanine, 2002; Peemans, 2013 et 2000. 
tialisées de ces différents récits urbanistiques. Dans ce contexte, l'espace dit public n'est devenu que l'espace résiduel entre le bâti et les infrastructures.

Cette situation est particulièrement présente dans les pays où les acteurs du secteur public sont faiblement actifs dans la production urbaine, ou lorsque leurs actions sont sectorielles et non concertées. C'est le cas par exemple en Algérie, où chaque acteur du secteur public agit suivant ses propres programmes, sur ses propres espaces et avec ses propres budgets. Il n'y a que très peu de coordination, voire parfois aucune, avec les actions des autres secteurs et, de manière générale, avec celles des autres acteurs, publics ou non. De plus, la libéralisation économique qu'a connue l’Algérie, comme de nombreux pays au cours des trente dernières années, a permis l'émergence de nouveaux acteurs, notamment privés, dans la production du territoire. Ces derniers ont également contribué à augmenter la fragmentation des villes par des réalisations de plus en plus isolées des tissus existants.

\section{L’espace public: un médiateur}

Dans nos contextes citadins contemporains, la construction du «vide» comme objet urbain devrait être une préoccupation importante. Elle permettrait certainement de retrouver des connexions et des liens, non seulement entre les différentes parties des territoires urbains mais aussi entre les acteurs de ces territoires. En ce sens, nous défendons l'idée que les récits d'un nombre important de villes devraient être des récits d'espaces publics comme fabricants de liens et de médiation. Dans cette optique, les espaces publics seraient au cœur de processus de coproduction urbaine. Ils permettraient de relier et de coordonner les actions sur la ville et de construire de la médiation entre les acteurs, afin de les mener vers la construction commune du territoire.

Pour ce faire, l'espace public doit assurer son rôle d'objet commun construit collectivement. Il doit alors dépasser son simple statut juridique qui ne garantit pas pour autant sa publicité. 
Celle-ci dépend essentiellement de la manière dont il est reconnu comme une des entités partagées, qui forment «la chose publique» et qui sont objet permanent de débat [...] Autrement dit, l'espace n'existe comme bien commun qu'en fonction de ce que les citoyens décident collectivement d'en faire (Declève, 2002: 70).

C'est donc grâce à cette action collective que l'espace public se construit en tant que tel, au fil d'ajustements constants. Les échanges sont continus entre les attentes et les représentations de chaque citoyen/acteur, et entre les objets de l'espace physique.

Commençons par l'espace physique. Comme on l'a expliqué précédemment, l'espace public constitue l'espace interstitiel entre les différents espaces privés, il porte intrinsèquement un potentiel de liaison. Les différents fragments du territoire, isolés les uns des autres, peuvent être reliés entre eux par l'espace qui leur est commun. Pour y arriver, il faut aménager ces espaces, et que les espaces privés, jusquelà isolés les uns des autres et de l'espace public, participent de cet aménagement. Par exemple, les configurations spatiales des clôtures autour des édifices publics ou privés ne permettent pas forcément un bon contact avec l'espace public et renforcent l'isolement de ces fragments. De ce fait, pour fabriquer des liens spatiaux à travers la production de l'espace public, il est impératif d'intervenir à la fois sur l'espace public et sur les configurations spatiales des espaces privés.

Cela nous mène directement à la question des jeux d'acteurs et de la production du territoire. Pour que l'espace public puisse jouer son rôle d'objet commun, il doit faire l'objet d'une construction commune. Cette dernière implique des transactions constantes entre les acteurs, qui les forcent parfois à céder une part de leurs acquis. C'est donc sur ce plan qu'intervient la médiation urbanistique, afin de mettre d'accord les acteurs autour de la fabrication de l'espace public devenu objet de médiation.

Si nous considérons la ville comme le bien commun de ses habitants, le projet de ville et de territoire que construit l'urbanisme devrait lui aussi être un projet commun. Il impliquerait, de ce fait, une coproduction du territoire qui nécessite inévitablement des pro- 
cessus de médiation entre ses acteurs et devrait aussi permettre de coordonner leurs actions et d'anticiper, de résoudre, voire d'éviter, les conflits éventuels. L'espace public comme objet de médiation pourrait être au centre des projets de ville contemporains et apporter des solutions concrètes à différents aspects du développement urbain.

\section{Les espaces publics du tramway d'Alger}

Afin d'illustrer davantage ces arguments et de montrer l'importance de penser l'espace public comme fabricant de liens, explorons brièvement le projet du tramway d'Alger. Revenons aux récits urbanistiques évoqués plus haut. Ces derniers se construisent, comme nous l'explique Corboz (2001), entre lecture et écriture des phénomènes en présence. Les acteurs du territoire portent un regard sur la ville en interprétant ses phénomènes en fonction de leur vision du monde, de leurs propres stratégies et de leur volonté d'action et de transformation de l'espace. Ils fabriquent donc continuellement des récits urbanistiques qui sont toujours entre interprétation et vision de projets. À Alger, ces récits ont été porteurs, depuis plus de cinquante ans, d'une lecture du territoire comme espace fragmenté résultant des différentes périodes de transformation de la ville. Il s'agit d'une lecture bien réelle du territoire que portent à la fois les professionnels de l'urbanisme et les acteurs politiques et gestionnaires de la ville. Il en résulte des projets qui visent, entre autres, à dépasser cette fragmentation. Cependant, ces récits de projet ont agi comme des métaphores autoréalisatrices (Meunier, 1998) en produisant à leur tour de la fragmentation urbaine. Les différentes stratégies sectorielles, l'interruption des processus de mise en œuvre des projets et l'apparition de nouveaux acteurs, que les récits urbanistiques officiels n'ont pas pris en considération, sont autant d'éléments qui ont contribué à renforcer l'idée et la réalité de la fragmentation urbaine.

Le cas du tramway d'Alger est assez représentatif de cet état de fait. Le ministère des Transports, initiateur du projet, propose une infrastructure de transport censée relier la périphérie est d'Alger au centre- 
РнОTO 1.1

\section{Les espaces du tramway d'Alger}

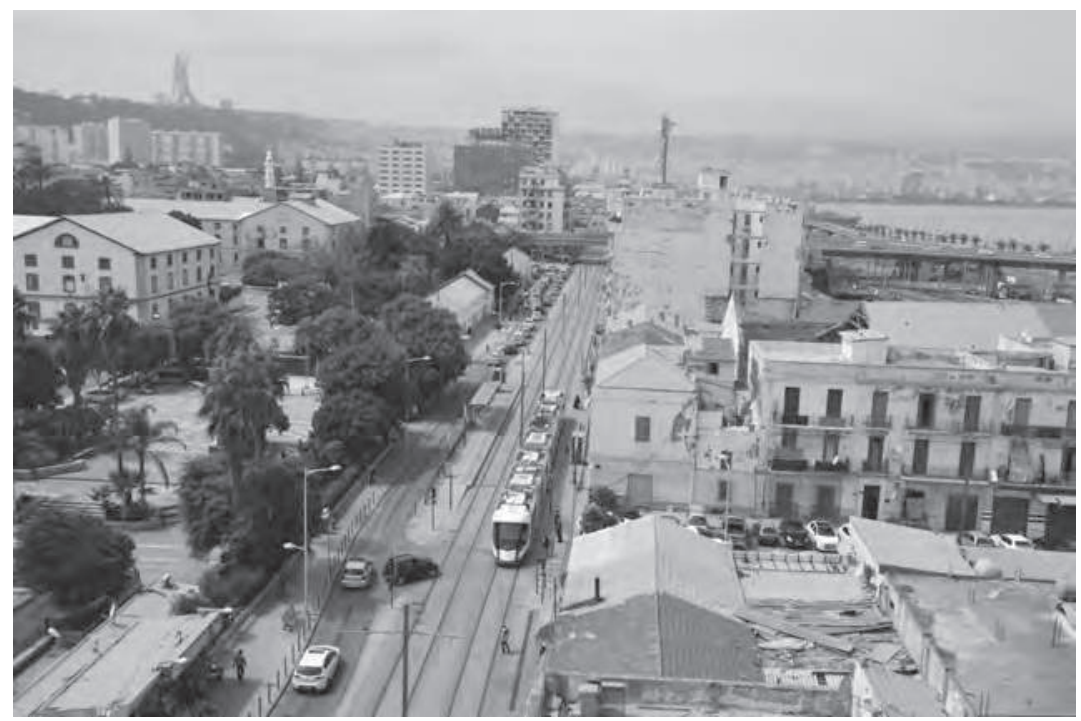

Photo: Annis M. Mezoued

ville. L'objectif est de désengorger l'autoroute du front de mer et de relier les fragments dispersés sur le plateau agricole de l'Est algérois, dédié essentiellement au logement, avec le centre, où se situent l'emploi et les services. Il s'agit donc d'un projet métropolitain, centralisé et sectoriel. Il y eut très peu de coordination entre les différents secteurs et avec les acteurs à l'échelle locale. Il en résulte un projet déconnecté spatialement des quartiers qu'il traverse. Les espaces publics que le tramway fabrique ne dépassent que rarement les limites de son emprise et créent parfois des dispositifs de sécurité qui isolent les aménagements de la vie locale. De ce fait, le tramway accentue à première vue la fragmentation existante et en crée de nouvelles.

Lorsque l'on s'en tient aux discours des acteurs urbanistiques algérois et à l'observation de leurs productions, souvent localisées dans les limites des propriétés ou de l'emprise des projets officiels, il devient difficile de dépasser le constat dominant de la fragmentation urbaine et de l'absence de lien entre les acteurs. Cependant, si l'on s'attache à 
identifier ce que raconte la ville d'elle-même, en essayant d'effectuer la synthèse des mille et un récits des «arts de faire au quotidien » dont nous parle merveilleusement bien Michel de Certeau (1990), nous notons que les récits urbanistiques portés par les acteurs producteurs de la ville s'enchevêtrent et s'hybrident pour raconter autre chose que ces récits eux-mêmes. En d'autres mots, la fragmentation que génère le tramway d'Alger n'est plus la seule histoire que raconte la ville. À partir d'analyses de la morphologie urbaine, des jeux d'acteurs et de leurs pratiques, nous observons çà et là des restructurations et des conformations d'espaces publics qui recréent de la continuité et qui font adhérer autrement l'espace de la ligne de tramway aux situations locales. Par exemple, les collectivités locales, peu prises en considération dans l'élaboration et la mise en œuvre du tramway, prennent spontanément le relais du projet en aménageant des espaces publics nouveaux tout au long de la ligne ou réaménagent les rues perpendiculaires au tramway. Elles fabriquent ainsi une continuité spatiale qui crée des liens entre la ligne et les quartiers qu'elle traverse. De même, certains acteurs privés ou des habitants profitent de l'arrivée du tramway pour prolonger la dynamique de transformation des bords de la ligne vers l'intérieur des quartiers, dont certains ont été construits de manière informelle et à la marge des récits urbanistiques officiels. Ils transforment le bâti par des densifications, transforment l'activité commerciale en s'adaptant à l'offre, aménagent parfois des trottoirs pour attirer les flux de voyageurs vers l'intérieur des quartiers, etc.

L'exemple du tramway, brièvement décrit ici, nous dit que la ville est capable, par l'intermédiaire de ses espaces publics, d'apporter des solutions à la fragmentation urbaine, même si elle ne résout pas l'ensemble des enjeux qu'elle suppose. Ce constat montre qu'il est possible de construire un autre récit urbanistique de l'avenir. Ce dernier ne serait pas le récit d'une personne ou d'un groupe ayant une certaine lecture de la ville et se projetant dans un avenir considéré comme idéal; mais plutôt un récit dont l'intrigue est déjà dans le présent par les dynamiques de l'enchevêtrement et de l'hybridation. Le socle de cet enchevêtrement, et c'est l'argument central ici, est bien l'espace public. 
РНОTO 1.2

\section{La requalification d'un quartier informel}

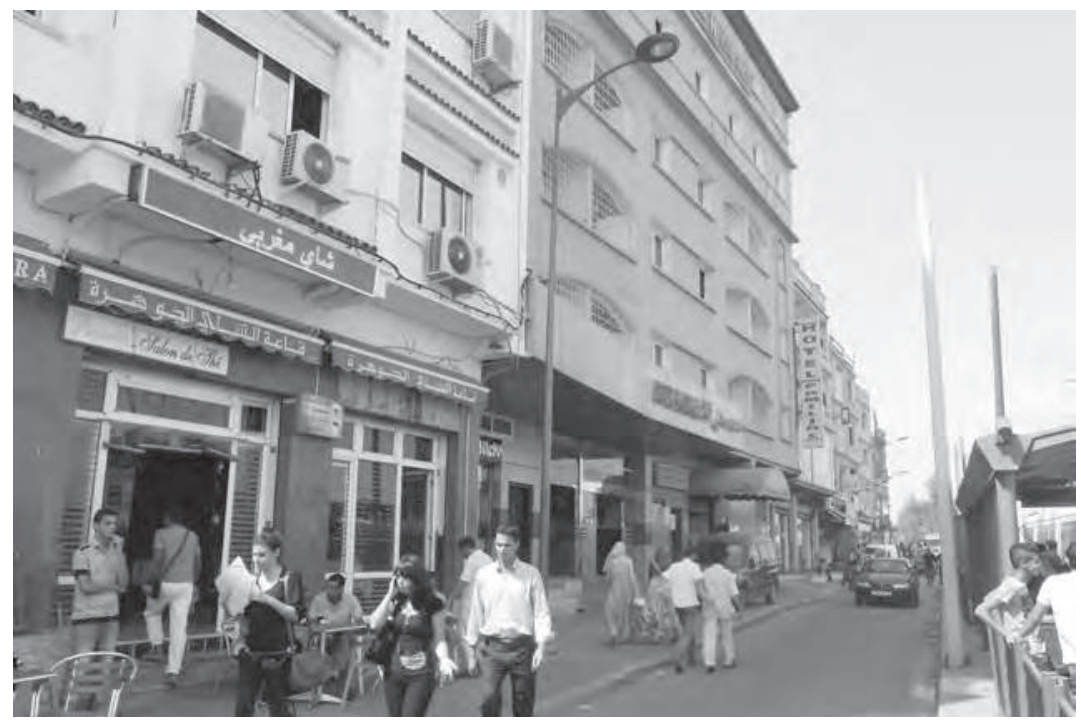

Photo: Annis M. Mezoued

Il paraît donc nécessaire que cet espace soit non seulement au cœur du projet de ville pour Alger, mais surtout qu'il soit pensé dans sa capacité à fabriquer du lien entre les fragments de la ville d'une part, et les acteurs qui les produisent d'autre part. La question importante qui se pose dans ce contexte est la suivante: comment faire de l'espace public un objet de préoccupation commun qui puisse servir de support à la fabrication de liens spatiaux et de médiation entre les acteurs? Le travail que nous avons réalisé sur Alger (2015) nous mène vers quelques pistes et suggestions sur lesquelles pourrait s'appuyer une coproduction de l'espace public.

La première hypothèse qui se vérifie par l'analyse des récits urbains algérois - à la fois des récits urbanistiques et du récit de la ville - est que des configurations spatiales et sociales créant des continuités dans l'espace public se superposent à la fragmentation urbaine et à l'absence de lien entre les acteurs et les échelles spatiotemporelles. Ces continuités constituent des signaux faibles d'un autre projet de 
ville, dont l'intrigue serait l'espace public comme fabricant de liens. Il est important de commencer par les repérer pour ensuite accompagner leur dynamique de transformation dans ce que Heurgon (2003) appelle une sorte de "prospective du présent» qui ne définit pas, comme la prospective classique, des tendances lourdes et un avenir probable, désirable ou improbable, mais plutôt un avenir souhaitable formulé à partir de l'existant. Rendre visibles ces signaux faibles constitue donc une étape importante.

Sur cette base, il est possible d'établir un projet spatial qui repose sur les dynamiques en place pour les accompagner et les renforcer. Dans le cas d'Alger, nous avions proposé un projet d'espaces publics qui permet de renforcer l'adhérence spatiale du tramway aux situations locales. L'idée est de prolonger les aménagements linéaires d'espaces publics pour construire une certaine épaisseur de part et d'autre de la ligne. Cette approche établit des continuités spatiales en permettant au piéton de rejoindre l'ensemble des points se situant à une distance confortable de marche à partir des arrêts du tramway. Elle favorise également l'établissement de «coutures» dans le tissu urbain en rétablissant des chemins rompus ou en créant de nouvelles continuités. Les projets des différents programmes sectoriels ne sont plus localisés en fonction des disponibilités foncières sur tout le territoire, mais en fonction de priorités liées à la présence d'espaces publics aménagés et connectés les uns aux autres.

Ce projet a servi de fondement à la discussion avec les différents acteurs du territoire, rencontrés individuellement, et avec certains habitants. Chacun a réagi au projet en fonction de ses propres stratégies. Contre toute attente, les intérêts des différents acteurs, qui pouvaient sembler divergents au premier abord, ont convergé. Même si certains ajustements restent à faire, le projet d'espace public, qu'on a présenté sous forme d'esquisse, semble faire consensus. Il a permis de rendre visibles les dynamiques en place, de mettre en avant le potentiel foncier et de souligner l'intérêt de créer le long de la ligne de tramway des espaces publics aménagés. Cette démarche a montré l'importance du projet d'espace public comme potentiel objet de 
médiation, autour duquel les discussions peuvent se mettre en place entre des acteurs qui n'entretenaient que très peu de relations. Ces derniers ont manifesté le besoin d'aller plus loin dans la proposition et d'organiser des tables rondes et des ateliers autour du projet. Cette idée n’a pas pu être réalisée pendant la recherche, mais n’a pas été abandonnée.

L'étape d'élaboration de l'objet de médiation et de son expérimentation n'est que l'amorce d'un long processus de médiation proprement dite. Même si les acteurs manifestent un intérêt pour le projet, le chemin reste long avant d'arriver à un consensus et à une concrétisation de la proposition. La médiation nécessite du temps, et des ajustements constants et continus. En conséquence, la dernière suggestion est d'accompagner le long processus de médiation par une prospective $d u$ présent productrice de liens.

Mettre les espaces publics au cœur du projet de ville pour Alger semble être une évidence. Leur potentiel de fabrication de liens spatiaux et de dépassement de la fragmentation existante, ainsi que leur capacité, en tant qu'objets collectifs, à constituer un espace de médiation, peuvent servir de base à l'élaboration d'un récit urbain portant l'idée de coproduction de la ville. Ce récit de l'avenir peut être celui d'Alger, mais peut tout aussi bien trouver écho à Ouagadougou, à Cochabamba, à Hanoi, à Bruxelles, ou ailleurs. 


\title{
CHAPITRE 2
}

\section{L'espace public et le patrimoine}

\author{
Pauline Bosredon
}

Les centres anciens occupent une place centrale dans l'évolution contemporaine de la fabrique urbaine et les dynamiques de renforcement des disparités sociospatiales en ville. Ils sont porteurs de valeurs tant symboliques qu'économiques, renvoyant aussi bien à l'enjeu très politique de construction territoriale et d'affirmation identitaire au moyen de leur patrimonialisation, qu'à celui de leur revitalisation socioéconomique (voir le sujet de la reconquête des centres-villes latino-américains). Dans ce contexte, le patrimoine urbain semble - de par sa définition même - investi d'un devoir de mixité sociale, d'échange et de partage. Mais sa valorisation traduitelle pour autant une coproduction de l'espace urbain impliquant l'ensemble des acteurs en présence et les multiples dimensions de leurs interactions (aménagement, pratiques, perceptions) ? La coproduction est ici comprise comme le résultat d'interactions d'acteurs multiples, de représentations diverses, d'appropriations ou de pratiques quotidiennes, tributaires aussi des politiques publiques et du cadre légal de la fabrique urbaine. Comprendre les dynamiques de coproduction dans un contexte de patrimonialisation implique donc de mettre en lumière les conflits et les rapports de force qu'elles impliquent et de dépasser l'idée trop souvent admise que le patrimoine représente le produit de valeurs historiques, culturelles et sociales consensuelles. 


\section{Un bien commun}

Considérons tout d'abord l'espace public comme un bien commun, dont l'accès est gratuit et ouvert à tous, que chacun peut s'approprier dans la mesure où il ne gêne pas la présence des autres ni leurs pratiques. C'est en théorie un espace partagé que l'on peut aussi définir par la négative: il n'est pas privatisé et son accès n'est pas restreint à certains groupes. Dans l'exemple de la Praça da Estação, ou place de la gare, à Belo Horizonte (Brésil), nous sommes face à un espace à la fois patrimonial (il fait partie d'un ensemble architectural classé) et public. La place de la gare est en effet une esplanade ouverte et c'est historiquement un lieu de rassemblement, au centre duquel on a érigé une statue à la gloire de l'Inconfidência Mineira, en hommage à la révolte en 1789 des Mineiros - habitants de Minas Gerais - contre la domination portugaise.

Plusieurs éléments entrent toutefois en contradiction avec la double qualité patrimoniale et publique de cet espace. Depuis 2010, une succession de décrets municipaux va dans le sens de sa fermeture et de son contrôle étroit. On privatise ponctuellement la place au moyen d'un accès payant et restreint et on y interdit les rassemblements populaires, en dehors d'un cadre strict fixé par la Municipalité. Par ailleurs, on a concédé le bâtiment de l'ancienne gare, autrefois lieu public, aujourd'hui patrimoine municipal classé, à une fondation privée qui y a ouvert un musée en partie payant.

Ces éléments, directement liés à la conjonction des intérêts publics (la Municipalité) et privés (la fondation), concourent à l'affaiblissement de la qualité publique de cet espace. Nous pouvons relire ce processus à la lumière des régimes de publicitél proposés par Staeheli, et Mitchell (2007). Sous l'angle des relations de propriété, la place et la gare demeurent des propriétés publiques. La première appartient à la Municipalité de Belo Horizonte et la seconde à la Compagnie brésilienne des chemins de fer urbains. Au regard des normes sociales,

1. Afin d'affiner l'appréciation du caractère plus ou moins public d'un espace, Staeheli et Mitchell proposent de considérer trois grands critères: les relations de propriété, les dynamiques sociales et les logiques de légitimation politique. 
certains usages comme l'appropriation des espaces ombragés par les sans-abri, l'organisation de duels de hip-hop le vendredi soir ou encore les rassemblements évangélistes gênent un certain nombre d'acteurs, en particulier la fondation privée du musée et les autorités municipales, qui voudraient que ces pratiques soient limitées. Enfin, les intérêts de ces protagonistes ont convergé pour aboutir à une réglementation contraignante de ces espaces, ce qui constitue une forme de légitimation politique de la réduction de leur publicité.

Et pourtant, à la suite de nombreuses manifestations organisées par le mouvement contestataire des artistes-activistes de la Praia da Estação (il s'agissait de transformer la place en une plage de centreville), une nouvelle régulation négociée de cet espace a vu le jour, ce que l'on peut assimiler à la coproduction d'un espace nouveau tant sur le plan symbolique (un espace de résistance à la privatisation et à la ségrégation sociale) que sur le plan des pratiques. Les manifestants ont réclamé l'accès libre et sans restriction à un espace auquel ils ont rendu son caractère public et patrimonial. Les pouvoirs publics tolèrent finalement cette situation. Cette relative tolérance et la discrétion de la police, qui n'est plus guère intervenue passé la première année de manifestations, s'expliquent en grande partie par le contexte de campagne électorale en 2012, le maire de Belo Horizonte ne souhaitant pas s'opposer trop directement à un mouvement bénéficiant d'une large couverture médiatique. En trois ans d'existence, la Praia a permis l'émergence d'un véritable espace politique d'expression et de revendications multiples.

Dans le cas de Ramallah (Territoires palestiniens occupés), une attention particulière est accordée par les politiques municipales à la réappropriation des espaces publics de la vieille ville. Le quartier historique, qui se trouve en position péricentrale vis-à-vis de l'actuel centre économique et fonctionnel de Ramallah, a en effet progressivement été délaissé, à la fois par ses habitants et par les édiles municipaux à partir de 1967, au début de l'occupation israélienne. Mais, depuis la fin des années 2000, c'est-à-dire depuis la fin de la seconde Intifada, ses espaces publics abandonnés, ses places et ses rues désertées connaissent un 
renouveau, dont le moteur est la politique municipale en faveur de la réhabilitation du patrimoine bâti, de la requalification des espaces ouverts de la vieille ville et du développement d'activités culturelles. À nouveau au cœur des préoccupations de la Municipalité de Ramallah après des années de peur et de couvre-feu, l'espace public est devenu un patrimoine commun à réinvestir, notamment car il incarne la résistance à l'occupation. Le quartier historique devient le théâtre d'un retour à la vie et aux activités culturelles. La réalisation de fresques sur les façades est par exemple l'un des outils utilisés par le Tamer Institute, centre culturel soutenu par la mairie, pour encourager les enfants, et par extension leurs parents, à participer à leur programmation d'activités culturelles et éducatives, à occuper les espaces ouverts et à y affirmer leur légitimité et leur appartenance à la ville. La multiplication des festivals, organisés dans les rues de façon récurrente, comme Wein a Ramallah (créé en 2010) ou Nouwar Nissane (créé en 2012), est une autre modalité de la patrimonialisation de la vieille ville par la réappropriation de ses espaces publics, visant in fine à rehausser l'attachement de la population de Ramallah à son histoire.

\section{Un objet de médiation}

Dans l'exemple de la ville palestinienne d'Hébron, le contexte est celui d'une fragmentation forte des espaces urbains marqués par une séparation entre des espaces interdits aux Palestiniens (les espaces des colons), d'autres qui sont la plupart du temps fermés aux colons, et des espaces partagés sous tension. Les rapports de force qui parcourent ces espaces - physiques et sociaux - mettent en jeu les militaires israéliens, les colons armés et protégés par les soldats, les habitants et les commerçants palestiniens, la Municipalité palestinienne d'Hébron, le Comité de réhabilitation d'Hébron (HRC)², plusieurs associations pacifistes palestiniennes et internationales, les

2. Le HRC (Hebron Rehabilitation Committee) a été créé à l'initiative de Yasser Arafat, alors président de l'Autorité palestinienne, pour réhabiliter le patrimoine bâti de la vieille ville d'Hébron et y faire revenir des habitants. 
touristes et les observateurs internationaux. La particularité de la ville d'Hébron est d'être un lieu de mémoire pour les trois religions monothéistes, du fait de la présence en son sein du caveau des Patriarches et, en particulier, du tombeau d'Abraham/Ibrahim, vénéré aussi bien par les musulmans que par les juifs. Depuis la guerre des Six Jours (1967) et les annexions territoriales israéliennes, de nombreux colons sont venus s'y installer par militantisme religieux, ce qui a mené à la constitution de la grande colonie de Kyriat Arba aux portes d'Hébron et à la mise en place de quatre colonies au cœur même de la vieille ville. Avec la présence de colons juifs appelant celle de soldats israéliens pour assurer leur protection et surveiller la mosquée d'Ibrahim, les tensions sont devenues très vives et la situation, intenable. C'est dans ce contexte que fut signé en 1997 le Protocole d'Hébron qui divise la ville en deux zones, $\mathrm{H}_{1}$ et $\mathrm{H}_{2}$, la seconde (où vivent 40 ooo Palestiniens et environ 600 colons) étant sous contrôle de l'armée israélienne: les 1500 soldats assurent la protection des colons.

Dans la vieille ville, située en zone $\mathrm{H}_{2}$, l'occupation des espaces privés (les maisons, les boutiques) représente un enjeu extrêmement fort où la patrimonialisation apparaît comme un levier efficace d'occupation et de légitimation. Les espaces publics deviennent alors les seuls espaces de rencontre possibles, comme en témoignent de nombreuses confrontations et tensions.

Le processus de patrimonialisation palestinien est mené depuis la fin des années 1990 par les acteurs publics (Municipalité et HRC). Il se décline en deux projets phares: la candidature de la vieille ville à l'UNESCO et la réhabilitation physique des maisons accompagnée d'un programme de relogement de Palestiniens dans la vieille ville. Ces deux opérations s'inscrivent dans un esprit de résistance à une menace clairement définie. Celle-ci n'est pas l'outrage du temps qui passe ni la négligence des contemporains face à l'héritage du passé, mais bien la guerre et ses belligérants, ou dans ce cas précis, la présence des colons et de l'armée israélienne. La reconnaissance du patrimoine culturel d'Hébron (en particulier à l'échelle internationale) représente pour les Palestiniens un outil de reconnaissance de leur légitimité, de 
leur mémoire et de leur histoire. La réhabilitation des maisons relève donc surtout d'un acte politique: rester ou revenir dans la vieille ville, c'est occuper l'espace et résister à la colonisation. La patrimonialisation concourt également à la production d'un discours performatif sur l'identité palestinienne de la vieille ville, sans cesse remise en cause par les colons juifs d'Hébron (notamment dans des affichages publics de propagande) et par l'État d'Israël. Ce dernier annonçait, en 2010 et par la voix de son chef de gouvernement, l'inscription du tombeau des Patriarches sur la liste de son patrimoine national.

Pour les habitants palestiniens comme pour les colons, les obstacles et les fermetures qui segmentent la vieille ville conduisent à une différenciation et à une hiérarchisation des espaces. Palestiniens, colons, touristes et observateurs internationaux s'y croisent, jamais loin du regard d'un soldat armé. Au cœur d'un patrimoine disputé, ces espaces publics de tension, partagés de force, deviennent aussi les seuls espaces possibles de médiation entre les parties opposées, où les acteurs internationaux (organisations non gouvernementales, touristes, observateurs des Nations unies) jouent le double rôle de témoins et d'intermédiaires.

Cela dit, d'autres espaces ouverts de la vieille ville d'Hébron sont aussi le théâtre d'affirmations identitaires visibles, tant dans les discours que dans des signes plus discrets. Dans les secteurs exclusifs (que ce soit envers les Palestiniens ou les colons), les façades, les murs de parpaing et les rideaux de fer des boutiques fermées sont la toile où s'expriment les identités, la résistance à l'occupation, le sentiment d'injustice, d'oppression et d'enfermement, les symboles nationaux, etc. Fresques, graffitis, drapeaux et affiches de propagande à destination des visiteurs sont autant de manières de marquer un espace revendiqué, réclamé pour soi et refusé à l'autre: cet espace soumis à des patrimonialisations concurrentes et des conflits d'appropriation ne peut être ici pleinement reconnu comme un espace public. 


\section{Un produit de la patrimonialisation}

Finalement, les processus et les politiques de patrimonialisation conduisent-ils à la production d'espaces partagés et consensuels? À l'inverse, sont-ils générateurs de conflit et de ségrégation, selon les modalités de la gentrification, de la relégation/marginalisation, voire de l'exclusion? Si la patrimonialisation est souvent assimilable à une reconquête des centres historiques, au profit de qui cette dernière s'opère-t-elle? Qu'en est-il de l'accès aux ressources et aux possibilités qu'offre la ville à tous, notamment aux populations les plus vulnérables? Ces dernières sont celles pour qui cet accès à la ville est le plus difficile, freiné, entravé, que ce soit par des raisons économiques (difficultés d'accès au foncier, relégation périphérique, statut d'occupation précaire), des raisons politiques (liées au contexte d'occupation dans l'exemple des villes palestiniennes) ou des raisons de capital social et culturel (notamment dans le cas de la place de la gare de Belo Horizonte). Ces populations montrent pourtant leur capacité d'adaptation aux pressions qui les fragilisent. Leurs stratégies et tactiques de contournement et d'adaptation (qu'elles soient individuelles ou collectives, plus ou moins conscientes et organisées) peuvent parfois être vues comme des formes de résistance. Celles-ci ne représentent cependant pas une lutte réactionnaire contre le changement: elles servent à faire face à ces pressions et à composer avec elles. Les capacités d'action des individus et des groupes dépendent grandement des divers capitaux qu'on a évoqués plus haut (économique, social, culturel), mais aussi des représentations, des parcours de vie et des rapports de pouvoir, ce qui renvoie plus largement aux luttes urbaines.

En théorie, espaces publics et espaces patrimoniaux devraient se superposer et se faire écho, car ils ont en commun les notions de partage, de consensus, de mixité des usagers et de diversité des pratiques. Pourtant, à la lumière de ces exemples, on voit clairement que des récits ${ }^{3}$

3. Nous empruntons le terme de «récit» à Aniss Mezoued (voir le chapitre $1 \mathrm{du}$ présent ouvrage), au sens d'œuvre de synthèse - lue comme un tout cohérent - entre les logiques des différents acteurs en jeu et les modes de production du territoire. 
de patrimonialisation concurrents, voire contradictoires, œuvrent à la production d'espaces de tension - voire en conflit ouvert - dont le caractère public revendiqué par certains groupes est contrarié par d'autres. Parfois, une synthèse s'opère, comme avec la Praça da Estação à Belo Horizonte, où les récits portés par la Municipalité et par le mouvement de la Praia s'accordent finalement et contribuent à la production d'un espace public négocié et donc partagé. Mais lorsque les récits paraissent irréconciliables, comme c'est le cas à Hébron où s'affrontent des patrimonialisations concurrentes visant à une appropriation exclusive de l'espace, les espaces publics semblent, dans une certaine mesure, pouvoir servir d'espaces de médiation et de soutien, sinon à un projet de ville commun, du moins à la rencontre et aux échanges entre les acteurs-belligérants. 


\title{
CHAPITRE 3
}

\section{Les revers du budget participatif}

\author{
Cécilia Montoya Antich
}

La participation citoyenne est devenue un enjeu important de la production urbaine contemporaine, particulièrement au sein des villes non occidentales. En urbanisme, elle renvoie à l'implication des citoyens dans la sphère publique et politique de l'aménagement urbain et de leurs espaces de vie. Dans ce contexte, les espaces publics sont généralement perçus comme des éléments clés pouvant favoriser la participation citoyenne. Dans certains cas, celle-ci ne porte pas seulement sur l'utilisation et la pratique des espaces publics, mais aussi sur leur production, afin notamment de mieux adapter les lieux produits aux envies, aux attentes et aux besoins des habitants, qui en sont aussi les premiers utilisateurs. Cette participation vise par ailleurs un plus grand engagement citoyen au sein des espaces de vie; la création d'espaces publics grâce aux budgets participatifs s'inscrit tout à fait dans ces objectifs et ce chapitre propose d'aborder cet aspect particulier, mais qui se popularise rapidement, de la production urbaine ${ }^{1}$.

1. Les budgets participatifs sont généralement considérés comme des outils propices à la participation citoyenne, à la démocratie et à la transparence. Démarche apparue pour la première fois à Porto Alegre (Brésil) en 1989 et largement généralisée après le premier Forum social mondial de 2001 tenu dans cette même ville, elle est aujourd'hui devenue un mécanisme primordial de gouvernance et de participation citoyenne au sein des espaces urbains. 
Le budget participatif est un outil de gestion municipal qui veut aider à la mise en place de projets urbains proposés par les habitants et financés (du moins en partie) par les institutions territoriales, bien souvent décentralisées. En conséquence, les personnes qui vivent dans la ville, et plus particulièrement dans le quartier, peuvent participer directement à la création d'espaces urbains. Ces initiatives ont souvent pour objectif de favoriser la construction d'espaces publics, de petites infrastructures destinées aux besoins des habitants, mais aussi de réduire les conflits d'usages et le manque d'appropriation de certains de ces espaces. Ces objectifs généraux s'appuient souvent sur différentes initiatives de budget participatif visant à répondre au manque de moyens des municipalités ou des entités territoriales décentralisées, ainsi qu'à augmenter l'implication des citoyens. Ces mécanismes de financement concernent aujourd'hui de nombreuses villes dans le monde et sont largement utilisés au sein des pays dits «en développement». À Lima, au sein des municipalités ayant peu de moyens financiers, les budgets participatifs sont bien souvent des procédures très attrayantes pour la population, qui voit en eux une façon de dynamiser l'aménagement du quartier.

La notion d'accessibilité représente ici un enjeu de première importance. En effet, les espaces publics sont, en théorie, des espaces intégrateurs par l'intermédiaire desquels les citadins participent de manière plus active aux affaires collectives. En ce sens, les espaces publics s'opposent aux espaces privés, et bien souvent aux usages privatisés des espaces urbains en général. À un autre niveau d'analyse, le caractère inclusif des espaces publics est généralement mobilisé pour la conciliation et la résolution de conflits, dans une optique de cohésion citoyenne (ceci implique, par exemple, l'engagement citoyen dans les affaires publiques locales, ou encore une bonne relation électeurs/élus). Dans certains districts de Lima cependant, la mise en place de budgets participatifs pour la création d'espaces publics remet justement en cause, comme nous allons le voir, certains fondements de l'accessibilité de ces espaces. 


\section{Une loi nationale au Pérou}

Au Pérou, la loi sur les budgets participatifs ${ }^{2}$ suit les alignements actuels du processus de décentralisation, proposant ainsi un dispositif de financement concret de la participation citoyenne à l'échelon local. Cette loi définit une démarche mettant en relation la population et les entités municipales, notamment pour la création de projets d'aménagement. Si ces derniers peuvent être très variés, ils engagent tous la participation de la société civile (la participation du voisinage peut aller de la conception des projets à leur construction, en passant par leur financement).

La loi instituant le budget participatif est née au sein du district populaire de Villa El Salvador ${ }^{3}$, division administrative formellement créée en 1983. Ce district est le résultat d'une occupation irrégulière de 60 ooo familles sur les terrains de Pamplona en 1963. Cet espace se trouve au sud de la ville et borde le quartier aisé de Las Casuarinas ${ }^{4}$. Les habitants de Pamplona sont issus d'autres districts urbains de Lima et de provinces rurales. Villa El Salvador s'apparente donc à ce que les autorités péruviennes qualifiaient au cours des années 1960 d'invasiones (espaces envahis), un phénomène que le manque de logements et la croissance importante de la population ont largement favorisé. En conséquence, l'occupation informelle de terrains plats et non construits à proximité de la ville consolidée s'est largement accélérée au cours des années 1960. En 1966, un décret régularise toutes les «invasions» précédentes et permet l'émission de titres de propriété pour l'ensemble des ménages concernés.

2. La Ley marco de presupuesto participativo 28056.

3. Selon une étude menée par l'institut national de statistique et d'Informatique péruvien (INEI), le district de Villa El Salvador ne comporte aucun individu ayant un revenu mensuel per capita considéré comme "élevé» (c'est-à-dire supérieur à $686 \mathrm{CAD}$ environ). Quinze autres districts de l'ensemble métropolitain sont dans la même situation.

4. Las Casuarinas est un quartier fermé à l'intérieur du district plutôt aisé de Surco (en 2012, ce district possédait le budget municipal le plus important dans la métropole, ayant accueilli dès sa création des familles originaires d'autres quartiers aisés de la capitale). 
Face à l'absence d'une législation précise, le processus de titrisation a encouragé l'occupation spontanée de terrains à Lima, chaque fois plus organisée. C'est dans ce contexte que se produit l'occupation des terrains inutilisés de la zone de Pamplona. Face à l'ampleur de cette occupation et à cause de la proximité de ce quartier avec celui de Las Casuarinas, les ménages sont rapidement déplacés par l'État à $30 \mathrm{~km}$ plus au sud.

L'histoire de la création de ce district et le développement de ces organisations de base (comités de voisins) en font un territoire remarquable en comparaison des autres districts de Lima. En conséquence, il est devenu une véritable référence en matière d'autogestion. La conception des quartiers de ce district servira ainsi d'exemple à beaucoup d'autres occupations irrégulières qui suivront, ce qui facilitera leur propre régularisation. Le plan d'occupation de ces quartiers organise les logements de manière à laisser un espace libre pour la réalisation d'un espace communal entre seize parcelles homogènes (de même taille). L'objectif de cet espace vacant est de donner un espace physique (public) pour la mise en place d'infrastructures (place, bureaux, etc.) dont le voisinage aura besoin. Ce mode particulier d'occupation de l'espace a petit à petit créé de nouveaux besoins d'aménagement.

Cette situation particulière s'explique par la manière dont la population a obtenu les terrains et à cause du manque de ressources

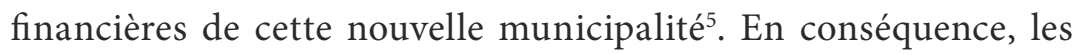
habitants ont rapidement dû prendre en main l'aménagement de leur quartier, de manière officieuse et sans compétence urbanistique autre que l'expérience de l'autoaménagement. Avec l'amélioration croissante des capacités aménagistes de la Municipalité de Villa El Salvador et face aux besoins et aux revendications de la population, le district décide, au cours des années 2000 , de convoquer les comités de voisins ${ }^{6}$ pour établir une liste des projets qu'ils voulaient voir se réaliser

5. En 2008 , le budget municipal de Villa El Salvador est à la $30^{\mathrm{e}}$ position (sur 43 districts) de tous les budgets municipaux des différents districts de Lima.

6. Les comités de voisins existent dans ce district depuis le moment où il a été habité. Les habitants avaient besoin de s'organiser en groupes de voisins, qui se sont 
dans leur quartier. Les comités de voisins intéressés devaient présenter un projet et y indiquer les personnes du quartier prêtes à s'investir, tout en spécifiant les modalités concrètes de cette implication. Cet investissement pouvait être d'ordre économique ou physique (en tant que main-d'œuvre principalement). L'initiative fut rapidement et facilement accueillie par la population résidente, qui était déjà organisée en comités de voisins actifs dans le développement du quartier. La généralisation des projets d'aménagement par l'intermédiaire des budgets participatifs à Villa El Salvador s'appuie ainsi sur des solidarités habitantes qui préexistaient à la mise en place d'outils participatifs légaux.

Les budgets participatifs représentent une véritable innovation au Pérou. Leur réussite à Villa El Salvador a largement favorisé la formalisation et la généralisation de cet outil municipal à l'ensemble de la ville et du pays. En 2001, le budget participatif devient un dispositif national, qui s'insère de surcroît dans les mesures de décentralisation prises par le gouvernement péruvien au cours de la même période. La formalisation des budgets participatifs et la généralisation de cet outil d'aménagement local posent cependant un certain nombre de questions. Lapplication de cet outil dans d'autres contextes que celui si particulier de Villa El Salvador mérite que l'on examine le caractère véritablement participatif de tels dispositifs. Plus précisément, ces projets favorisent-ils vraiment l'accessibilité des espaces publics? Pour répondre à cette interrogation, trois principes fondamentaux habituellement attribués aux espaces publics doivent donc être étudiés: leurs fonctions intégratrices, leurs usages non privatifs et leur rôle de conciliateurs (lorsqu'ils sont utilisés comme des espaces permettant aux habitants de faire valoir leurs envies et leurs objectifs, et lorsqu'ils sont le produit de cofinancements impliquant une entente citoyenne et une relation avec les gouvernements locaux).

convertis en comités, pour organiser l'accès aux lieux. Ces comités sont restés actifs pour effectuer les demandes des différents services urbains à la Municipalité (comme l'électricité et l'eau potable). Les comités sont encore très attentifs à l'évolution de l'aménagement de leur quartier. 


\section{Un rôle intégrateur mis en question}

La loi légiférant sur les budgets participatifs reste très souple. En effet, si elle décrit très précisément les étapes à suivre pour la mise en place du processus, la part du budget que les municipalités doivent engager n’est pas spécifiée. En conséquence, la participation financière des différents districts liméniens dans l'aménagement de leurs communes par l'intermédiaire des budgets participatifs présente d'importantes disparités. D'un bout à l'autre du spectre, le montant de la participation municipale peut être insignifiant (dans beaucoup des districts consolidés de Lima), ou s'élever, dans le meilleur des cas, à plus de $30 \%$ (dans les districts plutôt populaires et périphériques). Dans la capitale péruvienne, ce dispositif a plus de succès au sein des districts les moins bien aménagés et au sein des municipalités ayant le moins de moyens financiers. Si les budgets participatifs visent théoriquement une plus grande démocratisation de l'aménagement de l'espace au niveau local, ils peuvent aussi représenter un outil privilégié (et formalisé) pour l'aménagement des quartiers les plus précaires. Ainsi, les budgets participatifs instaurent d'importantes disparités entre les différents districts liméniens, qui ne s'appuient pas sur les mêmes outils pour aménager leurs territoires. Cette disparité se retrouve par ailleurs au sein d'un même district, où l'engagement financier des comités de voisins est loin d'être homogène. Les budgets participatifs favorisent donc parfois les disparités urbanistiques intramunicipales et intermunicipales.

Ces dernières sont par ailleurs renforcées par les modalités de financement des projets. En effet, les comités de voisins qui utilisent cet outil ont plus de chances d'obtenir de l'aide gouvernementale pour la mise en place d'autres projets participatifs, ce qui les pousse à le mobiliser. En conséquence, il y a un véritable effet multiplicateur qui accroît les disparités territoriales. Cette réalité est exacerbée par le manque de planification urbaine à l'échelle locale et par l'absence d'un établissement des priorités des projets d'aménagement. Les territoires urbains s'apparentent donc parfois à de véritables patchworks de quartiers différemment aménagés. 
À un autre niveau d'analyse, le budget participatif est aussi perçu comme un instrument d'aménagement qu'on réserve aux espaces les plus précaires et aux municipalités ayant le moins de moyens financiers. En effet, les projets de budget participatif sont généralement associés aux populations qui ont «envahi » des territoires de la ville et qui ont pratiqué l'autoconstruction et l'autoaménagement. La production d'espaces publics grâce au budget participatif peut donc favoriser une stigmatisation de certains quartiers et révéler les préjugés d'une partie de la société liménienne vis-à-vis des espaces précaires de la ville. En conséquence, une homogénéisation du nombre et de la répartition géographique des projets de budget participatif dans l'ensemble des districts apparaît aujourd'hui essentielle.

\section{L'aspect public de l'espace mis en question}

L'utilisation de budgets participatifs pour l'aménagement d'espaces locaux pose par ailleurs la question du caractère véritablement public des espaces que les communautés d'habitants ont créés. À Villa El Salvador, la généralisation de projets d'aménagement à budget participatif (qui ne sont pas nécessairement suivis et encadrés par la Municipalité) a engendré une semi-privatisation des espaces publics, ce qui s'oppose directement aux objectifs de démocratisation portés par un tel outil d'aménagement.

Les projets d'aménagement par budget participatif sont sélectionnés par la mairie. Pour faire son choix, elle évalue notamment le caractère public des projets proposés par les habitants et portés par les comités de voisins, et l'apport (surtout financier) qui pourra être fourni par les habitants eux-mêmes pour la mise en place de l'aménagement (les municipalités disposant de peu de moyens techniques et financiers, plus la participation citoyenne est importante, plus le projet participatif aura de chances de voir le jour). Les apports proposés par les voisins vont alors au-delà de la simple proposition d'un projet. Les habitants des quartiers en question vont, dans certains cas, jusqu'à donner une partie de l'argent nécessaire à la construction de l'espace public en 
question. Lapport financier dépend des ressources économiques des habitants et des activités qu'ils ont mises en œuvre pour récolter le financement. Ceux-ci peuvent par ailleurs proposer de participer directement, en tant que main-d'œuvre, à la construction du projet. Ces deux types d'investissements - financier ou physique (et en temps) - favorisent l'attachement au lieu créé, ce qui peut se traduire par une semi-privatisation de l'espace par les habitants ayant aidé à sa construction. En effet, face au désengagement de l'État dans l'aménagement de ces quartiers et dans l'entretien des aménagements après réalisation, les habitants trouvent parfois légitime de s'approprier et de privatiser les espaces qu'ils ont eux-mêmes construits, voire financés.

\section{Une conciliation difficile}

À un autre niveau d'analyse, la construction d'espaces publics par l'intermédiaire des budgets participatifs est parfois mise à mal par l'hétérogénéité des compositions socioéconomiques des quartiers. Dans ces cas précis, ils peuvent devenir des vecteurs d'aggravation importants des disparités sociospatiales. Au sein des districts situés à l'extrême sud de Lima, l'investissement des habitants dans l'aménagement de leur espace revêt une dimension particulière. En effet, certains de ces districts disposent de budgets très limités, alors qu'ils constituent des espaces balnéaires particulièrement attractifs, qui accueillent des populations très diverses: des classes populaires résidentes à l'année, des classes plutôt aisées qui y vivent pendant l'été et des visiteurs journaliers non résidents. Pour ces municipalités aux faibles capacités de financement, en comparaison d'autres municipalités de Lima ${ }^{7}$, l'important afflux d'une population estivale soulève de nombreux enjeux d'aménagement urbain. Parce que ces municipalités sont toujours à la recherche de nouveaux moyens financiers pour aménager leurs territoires, les budgets participatifs représentent une solution concrète, qui répond par ailleurs aux attentes des classes

7. En 2012, les budgets municipaux des cinq districts étudiés font partie des six budgets les moins importants de tous les districts métropolitains. 
populaires installées sur ce territoire tout au long de l'année. Pour ces habitants, les budgets participatifs permettent le raccordement à l'eau potable, l'aménagement de trottoirs, ou encore la réparation d'une salle de collège. Face à cette mobilisation collective, la population estivale aisée a elle aussi décidé de s'organiser en comité de voisins et de faire des propositions d'aménagement sur les districts qu'elle occupe, en sachant que ce n'est que par la mise en place de budgets participatifs que ces populations réaliseront des projets dans leur quartier.

Les propositions d'aménagement de la population saisonnière sont révélatrices d'une vision particulière du territoire, car elles couvrent avant tout ses besoins récréatifs et esthétiques. Les demandes de ces deux populations distinctes divergent donc fortement, tout en représentant les deux faces d'un même territoire. C'est dans cette situation que s'observe par ailleurs l'importance du pouvoir de décision des maires, notamment accordé selon le cadre légal de l'aménagement du territoire. Au sein des quartiers que nous avons étudiés, les maires disposent d'une grande liberté, par l'intermédiaire du dispositif de budget participatif, notamment dans le choix des aménagements. Dans le cas des stations balnéaires étudiées, les maires ont favorisé, dès qu'ils en ont eu l'occasion, les projets qui allaient dans le sens du développement de la partie balnéaire du district (partie qui est plus intéressante pour eux, car elle attire plus d'investissements économiques), tout en sachant que ces aménagements ne bénéficiaient pas aux habitants permanents. Par ailleurs, les maires peuvent tout à fait se désengager d'un projet de budget participatif préalablement accordé, parfois au profit d'un autre. Il existe à ce titre un manque criant de moyens légaux mis à disposition des citoyens pour s'opposer aux choix des maires. De manière générale, les dispositifs de contrôle des projets choisis restent très limités. Les habitants ne peuvent ainsi faire prévaloir les accords préalablement passés avec le maire lors des séances de prise de décision. 
Les exemples présentés nous permettent finalement d'observer comment la mise en place d'espaces publics par l'intermédiaire des budgets participatifs peut conduire à des situations qui mettent en cause leur accessibilité. Malgré les bonnes intentions affichées par ce type d'outil urbanistique pour faire participer les habitants dans la planification et l'aménagement de leur espace de vie, certains territoires se trouvent plus stigmatisés, la fragmentation urbaine s'accroît parfois et les espaces publics peuvent faire l'objet de formes de privatisation. Par ailleurs, le cadre légal déficient peut favoriser les situations de conflit, notamment lorsque les intérêts des autorités locales entrent en contradiction avec ceux des habitants, qui disposent finalement d'une faible marge de manœuvre décisionnelle et de peu de possibilités de recours légal. Nous croyons en conséquence que la consolidation du cadre légal existant permettrait de prévenir certains revers et abus. 


\title{
CHAPITRE 4
}

\section{L'école coproductrice de la ville et du projet de société}

\author{
Valeria Cartes Leal
}

Objet singulier pour l'urbaniste et l'aménageur, l'école représente un espace clé de la construction citoyenne. Cependant, l'école reste souvent cantonnée à sa vocation première, l'éducation des enfants, sans faire l'objet de réflexions plus larges portant sur le rôle qu'elle pourrait jouer dans la mise en œuvre d'initiatives urbanistiques plus inclusives.

À partir de 2006 au Chili, le problème de l'éducation s'est retrouvé au cœur d'une explosion de la grogne sociale. Les problèmes larvés du système éducatif ont remis en cause ce que de nombreux gouvernements nommaient la réussite du modèle chilien. Une série de manifestations organisées par les élèves du secondaire a donné lieu à ce qu’on a appelé la Revolución Pingüina ${ }^{1}$.

La révolution des pingouins a entraîné la mise en place d'un conseil consultatif sur l'éducation, chargé d'élaborer un nouveau cadre institutionnel pour l'éducation chilienne et de préparer une loi générale. Malgré ces tentatives, de nombreuses organisations - d'élèves pour la plupart - ont rejeté la nouvelle loi, provoquant une série de manifestations. Les élèves du secondaire accusaient l'État de se désintéresser

1. Le terme "pingouin» renvoie à l'uniforme des élèves du primaire et du secondaire. 
des problèmes sociaux; les étudiants du cycle supérieur réclamaient de meilleures conditions de financement et un accès plus souple à l'enseignement; les lycéens, quant à eux, revendiquaient la gratuité et de meilleures conditions matérielles. La communauté scolaire dans son ensemble dénonçait la médiocrité de l'enseignement, la faiblesse des investissements publics, l'autoritarisme de l'État en matière de politique éducative et l'état psychologique des enseignants.

Les revendications des élèves et des étudiants chiliens dépassaient les frontières du Chili et voulaient réexaminer plus largement les liens entre éducation et citoyenneté à l'échelle du continent latinoaméricain. Les problèmes du système scolaire sont devenus une priorité du programme politique du gouvernement. La réforme des politiques publiques urbaines et d'éducation ainsi qu'un projet de réforme fiscale occupent toujours le centre des discussions publiques. Cependant, les projets mis en œuvre manquent de transversalité et semblent timides au regard des enjeux sociaux beaucoup plus larges que le mouvement a révélés.

La situation du Chili illustre la place essentielle qu'occupe le système éducatif dans les sociétés contemporaines. L'école peut ainsi occuper une place centrale et stratégique dans l'espace public: plus qu'un lieu d'éducation formel, elle peut devenir un espace de mobilisation et de revendication tant social que politique. Dans ce contexte, comment réintégrer l'école au centre de projets de société et de l'espace public? Du point de vue de l'urbanisme et de l'aménagement, cette question en entraîne une autre, que ce soit sur le continent latino-américain ou en Europe de l'Ouest: comment l'école peut-elle être pensée comme un espace intégrateur et citoyen situé au cœur des projets de développement urbain?

\section{L'école et la fabrique urbaine}

Prenons comme point de départ la place de l'école dans le contexte européen et, plus précisément, en Belgique. Le XIX ${ }^{\mathrm{e}}$ siècle a vu l'école devenir un lieu d'activités éducatives gratuites obligatoires et un 
bâtiment public particulièrement lisible dans le tissu urbain. Avec la maison, elle fournissait le cadre physique et social nécessaire à l'apprentissage des enfants, car elle occupait un rôle de premier plan dans la vie de ceux-ci. De même, les écoles étaient localisées aux endroits le plus voyants et les plus exposés de la ville, sur d'importantes artères et autour des places publiques par exemple. Il n'y avait donc pas de réelle préoccupation quant à la distance ou à la nature du chemin que l'enfant devait parcourir deux fois par jour ni de prise en compte du caractère «agréable» de l'environnement urbain dans lequel l'école s'insérait.

La place de l'école dans la ville a finalement peu évolué au regard de l'ensemble des changements urbains, sociaux et éducatifs qui ont marqué les sociétés européennes depuis le $\mathrm{XIX}^{\mathrm{e}}$ siècle: celle-ci reste cantonnée à sa fonction d'espace consacré à l'éducation des enfants. Les préoccupations sociales des architectes dans les années 1970-1990, ainsi que le développement technologique, n’ont pas altéré la logique initiale de l'espace scolaire. Cela nous amène à réfléchir, d'une part, à l'environnement quotidien de l'école, considérant qu'il conditionne l'attachement ou le détachement de l'enfant à l'égard de l'environnement urbain extérieur, et d'autre part, au rôle de l'école dans la construction citoyenne.

Dans ce contexte, nous pouvons formuler l'hypothèse que les modèles et l'organisation des écoles doivent être pensés en fonction des besoins d'apprentissage du public qui les fréquente et de leur relation avec leur environnement social et spatial, dans l'objectif d'un enrichissement mutuel. En d'autres mots, pour que l'école contribue à la production de l'espace public, elle doit s'ouvrir à la communauté. Il devient alors nécessaire de surmonter la conception traditionnelle de l'école et de l'intégrer à d'autres sphères éducatives, bien au-delà de l'univers scolaire proprement dit. On doit considérer l'école comme un élément urbain dynamique, en interaction avec la réalité sociale et spatiale de la ville. De même, à supposer que la collectivité et l'environnement disposent de connaissances utiles à l'école, cette dernière pourra devenir une source de socialisation, d'apprentissage de la 
citoyenneté, ainsi qu'un puissant agent catalyseur et intégrateur de la communauté à la ville.

L'articulation entre l'école et la ville ne se fait pas seulement dans le cadre des infrastructures scolaires ou aux heures d'ouverture de l'école. La famille, le quartier, la ville, les équipements et les services urbains offrent aussi des ressources éducatives, qui contribuent à l'élaboration du contexte matériel et humain dans lequel viennent s'ancrer dans l'espace public les modèles éducatifs novateurs. Les relations entre l'école et l'espace public sont ainsi variées: elles concernent l'architecture du bâtiment scolaire, les accès à l'école, les activités programmées au sein de l'école ouvertes à la communauté, l'aménagement des rues et des espaces urbains qui l'entourent, la circulation, etc.; elles renvoient aussi aux écoles qui promeuvent l'engagement social et une ouverture au quartier et à la ville; elles touchent enfin les écoles qui voient dans l'environnement une source d'enseignement, et celles qui établissent un lien entre l'apprentissage à l'intérieur et à l'extérieur de l'école.

En outre, l'environnement et l'espace public de l'école sont aussi concernés par les enjeux socioculturels du quartier. C'est dans l'espace public que se croisent la programmation d'un projet urbain d'intégration et celle d'un projet scolaire, souvent à l'échelle du quotidien.

\section{Un rôle socialisateur}

De nombreuses sociétés conçoivent l'école comme un lieu d'administration et d'éducation. Cependant, l'école assume aussi un rôle de socialisateur majeur qui dépasse de loin le contexte éducatif. La relation entre l'école et le quartier concrétise le sens de l'urbanité et participe à l'éducation de la citoyenneté. On peut utiliser l'environnement de proximité de l'école comme un recours physique et de contenu, dont l'utilisation dépendra des caractéristiques propres et des particularités pédagogiques de l'école. Le quartier de l'école peut être défini comme un réseau de lieux, situés à proximité de l'établissement éducatif et dont l'occupation est marquée par la présence du 
public scolaire, mais aussi par les activités parascolaires. L'espace public de proximité associé à l'école se précise, plus ou moins, dans les relations d'usage qui ont lieu le plus souvent dans la rue: les achats avant de se rendre à la maison, les activités de loisir, le déplacement en soi, l'attente à l'arrêt de bus ou de tram, etc.

En urbanisme, la rue et l'espace public sont toujours évoqués comme les principales sources d'expérience urbaine de l'enfant et des jeunes. L'espace public offre en effet à ces derniers un certain nombre de stimulations qui favorisent leur développement et leur intégration dans le contexte spatial. Heidegger et Merleau-Ponty décrivent la rue comme le cadre matériel des expériences quotidiennes de l'homme. Le comportement des usagers, la sensibilité que l'espace évoque et sa morphologie contribuent à construire l'image que l'on se fait de la rue et de l'espace public. Cependant, construire l'espace public ne consiste pas uniquement à définir l'implantation des bâtiments ni à élaborer leurs façades. Il est en effet nécessaire de comprendre sa logique propre et ses réseaux, qui entretiennent les liens sociaux.

C'est dans l'espace public que se côtoient et s'articulent toutes les catégories sociales et les usages attachés à de multiples temporalités. À cet égard, les espaces publics d'une ville sont par essence des lieux de résolution quotidienne des relations de proximité-distance entre les différents membres d'une société. Simultanément, le territoire est composé par la présence de l'école et les pratiques qui lui sont associées et qui, à leur tour, contribuent à structurer l'espace public.

\section{Le parvis : une passerelle entre la ville et l'école}

Dans la rue, le trottoir ou stoep, comme cela se disait en néerlandais au XVII ${ }^{\mathrm{e}}$ siècle, est un espace multifonctionnel où les marchands et autres métiers se mettaient en scène. Ce trottoir, aménagé au ras des maisons, était légèrement surélevé par rapport à la chaussée et pouvait accueillir les jeux des enfants, sous l'œil des parents qui les surveillaient par la porte d'entrée. C'était aussi un lieu qui incitait à la rencontre entre voisins et passants, à la conversation et au repos sur les 
bancs disposés le long des façades. «De ce stoep partaient une ou plusieurs marches vers la porte d'entrée des habitations qui, par leur nombre et leur revêtement, montraient le degré de richesse de leurs occupants» (Loiseau, 2005: 6). Le flux, le bâti et le vécu - ces trois éléments - définissent l'espace de relation entre l'école et la ville, qui représente l'objet urbain fondamental de l'articulation entre espaces scolaires et espaces urbains. Cet espace intermédiaire, qui peut se matérialiser dans le parvis ou le chemin de l'école, devient le support de l'expérience de l'enfant et participe à son éducation.

L'expérience du déplacement d'un enfant entre la maison et l'école peut être décrite selon le chemin parcouru, le type de transport utilisé, le temps nécessaire, selon que l'enfant est seul ou accompagné sur tout le trajet ou sur une partie de celui-ci, et selon la perception de l'espace public. Cette chaîne des pratiques et des lieux montre les liens avec l'espace, incluant les éléments paysagers du trajet, entre le déplacement scolaire et l'organisation urbaine. La marche à pied, pour les élèves de tout âge, bénéficie d'une image positive, particulièrement quand il s'agit de "petites distances» qui permettent de "voir de nouvelles choses", ainsi que de rencontrer des personnes et de se faire de nouveaux amis. La notion de petite distance est évidemment relative: une de nos études menées sur Bruxelles montre en effet que les trajets à pied au primaire se font dans un rayon de $500 \mathrm{~m}$, tandis qu'au secondaire, ils peuvent atteindre $1,5 \mathrm{~km}$. Cependant, toute promenade est l'occasion de faire des découvertes et des observations qui stimulent la réflexion du marcheur et enrichissent les caractéristiques propres des lieux traversés. L'école représente un équipement urbain intermédiaire de l'espace public et un pôle d'attraction; elle sert de support, de scène, de lieu d'échanges, de rencontres et de discussions, où le contact social entre les élèves et les parents au moment de l'attente à la sortie joue un rôle important. En somme, la charge symbolique de l'école est celle d'un lieu qui amène l'habitant à faire face à son environnement et à son quartier. 


\section{L'ouverture de l'école à l'espace public}

Ce que nous venons d'évoquer montre l'importance du rôle de l'école dans l'intégration socioculturelle et spatiale des enfants. Pour que cela se produise, l'école doit s'ouvrir par ses pratiques pédagogiques et son intégration au milieu spatioculturel urbain. Pour ce faire, elle doit disposer de structures urbaines (espaces verts, équipements, espaces publics, transports, etc.) et d'un réseau organisé d'acteurs sociaux venant de la communauté scolaire comme de la communauté urbaine. Dans ce contexte, les espaces publics peuvent intégrer les enfants à la structure spatiale, culturelle et urbaine et ainsi participer au développement de la vie collective dans le quartier.

Le système éducatif joue ici le rôle de lien entre l'apprentissage de la citoyenneté et l'appropriation de l'espace public. L'urbaniste peut être en mesure de renforcer les rapports qu'entretient l'enfant avec l'espace public, pour lui permettre de se construire et de participer activement à la société. Établir une liaison étroite entre l'enfant et le territoire qu'il habite, c'est finalement participer au développement culturel et spatial de la société.

Il convient cependant de reconsidérer l'articulation entre les établissements scolaires et les espaces publics, de repenser le rapport entre l'enfant et la ville et de valoriser le potentiel éducatif de l'espace urbain, pour favoriser les processus de développement et d'intégration sociale et culturelle des élèves. Il est ainsi nécessaire d'intervenir non seulement dans l'aménagement ou l'organisation de l'espace public, mais également sur le plan de ses modalités d'utilisation. En ce sens, l'enfant doit avoir accès à toute la ville pour bénéficier du potentiel éducatif de l'espace urbain. Il faut lui proposer des modalités d'utilisation de cet espace pour qu'il ait un accès orienté sur des processus d'intégrations sociale et culturelle.

La notion d'urbanisme scolaire veut ainsi proposer de nouvelles réflexions quant à la place de l'école dans les approches et les théories urbanistiques. Cette notion et les approches évoquées précédemment illustrent le fait que l'école peut être plus qu'un lieu d'éducation formelle 
et devenir un lieu de mobilisation et de revendication. Plutôt que de voir cet espace jouer un rôle par défaut, comme dans le cas chilien, l'école pourrait occuper une place plus centrale dans les espaces publics urbains et devenir le moteur d'un projet de société, à condition qu'elle soit pensée en relation avec les projets urbains.

L'urbanisme scolaire implique la coopération entre les différentes fonctions urbaines et entre les outils et les dispositifs urbanistiques mis en place. C'est aussi une stratégie urbanistique qui intègre les acteurs locaux. Du point de vue de l'aménagement, l'urbanisme scolaire réfléchit aux divers problèmes relatifs à la répartition, à la programmation et à l'articulation des bâtiments scolaires dans l'ensemble de la ville. L'urbanisme scolaire élabore des stratégies et des actions allant de l'échelle architecturale du bâtiment scolaire à l'échelle métropolitaine.

Si l'école sert à la fois de déclencheur et d'acteur d'un projet urbain porteur d'une vision de société, comment peut-elle aussi jouer un rôle d'opérateur déterminant dans l'urbanisme? Comment ce concept d'ouverture et d'intégration entre l'école et la ville fonctionnerait-il dans le contexte chilien, où la demande émane directement de la population scolaire, où le système éducatif est beaucoup plus centralisé que le système bruxellois, où la dichotomie public-privé est très présente et où les échelles de territoire et la taille des établissements scolaires sont beaucoup plus importantes? Tant d'éléments et de problématiques qui font émerger des pratiques et des modes d'organisation spécifiques, que ce soit pour le ramassage scolaire, les activités éducatives et parascolaires, les nouvelles fonctions urbaines et les métiers.

Les espaces et les conditions d'usage offerts par la ville pour répondre aux enjeux d'éducation sont donc une question fondamentale à poser sur le plan urbanistique. Le concept d'urbanisme scolaire ouvre le champ des possibles d'un nouveau modèle d'école qui fait la ville. En tant que projet de société, il doit s'adapter à chaque contexte dans un projet urbain entendu comme processus de production de la ville ouverte et heuristique. 


\section{DEUXIÈME PARTIE \\ LA FABRICATION URBAINE AU PRISME DES JEUX d'ÉCHELLES}





\title{
CHAPITRE 5
}

\section{L'échelle dans la définition de l'urbain et de l'urbanité}

\author{
Mauricio Anaya
}

Chaque ville est unique, et possède en quelque sorte sa propre personnalité. Les manières par lesquelles chaque ville structure les différents niveaux de son tissu, et les différents échelons de ses instances de gestion, ne montrent pas seulement sa personnalité: elles révèlent aussi des situations, bien souvent contradictoires, résultantes de tensions entre les échelles locales et globales de la production urbaine. En prenant des exemples tirés d'une ville moyenne sud-américaine qui veut très fièrement devenir une métropole (car apparemment c'est le seul chemin à suivre), ce chapitre remet en question la manière dont les échelles (spatiales, physiques, temporelles, sociales, urbanistiques, etc.) qui la composent se mettent en relation et produisent des résultats particuliers.

\section{Le régime d'urbanité et la continuité urbaine}

De manière générale, les questionnements qui émergent des études sur la ville et sur la vie urbaine tournent autour de la compréhension des articulations entre les logiques de production, de conformation et de structuration de l'espace. À celles-ci se rajoutent bien souvent les processus d'appropriation, d'usage et de production des lieux. Pour reprendre une expression de Bernard Declève, ces logiques et ces 
processus représentent bien les articulations entre une «morphologique» et une «anthropo-logique». Dans cette perspective, certains regards portés sur la ville essaient d'aller au-delà de sa conception comme objet d'étude abstrait et appréhendable dans sa totalité, pour tenter de dialoguer avec elle en tant que sujet concret et phénomène vivant. L'urbain «est une abstraction, mais le contraire d'une entité métaphysique, une abstraction concrète, liée à la pratique » (Lefebvre, 1970: 159). L'urbain est donc à la fois unique et multiple, constitué de ce qui est individuel et de ce qui est collectif; on doit par ailleurs resituer la ville dans des échelles temporelles multiples et la considérer comme le produit d'une histoire, le résultat de routines et de quotidiennetés du temps présent, mais aussi comme une entité en devenir, tournée vers son avenir, comme projet. C'est dans cette logique qu'une meilleure compréhension des articulations complexes entre ses caractères - matériel et immatériel - peut nous aider à révéler quelques traces de la personnalité d'une ville particulière, c'est-à-dire du régime de la relation entre la société et l'espace qu'elle produit et qu'elle habite.

Le régime d'urbanité, soit l'expression momentanée de formes collectives de sociabilité (dans la même ligne que le «régime de lisibilité de la substance urbaine» que propose Lussault), exprime les manières dont une société locale produit des solutions, jamais absolues et en évolution permanente, à des situations que les relations conflictuelles génèrent entre l'espace vécu, celui des pratiques et des représentations, et l'espace social, celui des interactions sociales; les catégories d'acteurs qu'on considère comme urbanisantes et celles qu'on considère comme urbanisées; et les conditions de fonctionnement et de permanence des systèmes locaux et les conditions qu'imposent les systèmes globaux.

Aller à la rencontre de ce que nous appelons un régime d'urbanité, c'est faire face à tous les éléments du système urbain qui, au moyen de leurs interactions, lui donnent forme. Cette notion recouvre donc les degrés de continuité de l'urbain et de la ville en tant que «modèle de vivre ensemble» auxquels les articulations entre éléments et situations divers donnent lieu. 
À un autre niveau d'analyse, la continuité urbaine, c'est-à-dire les degrés d'articulation entre les éléments soutenant le régime, présentent deux volets fondamentaux. Le premier concerne les niveaux de continuité des composants de l'espace urbain, du point de vue de la structuration de l'espace physique et de la morphologie du tissu:

L'histoire des villes peut être pensée en termes d'échelles de l'espace urbain, du passage du local au global, de l'intime à la foule. La médina arabe, par exemple, déploie une série d'échelles successives entre le plus petit détail ornemental de ses carreaux de céramique; l'espace privé, secret, enclos et frais de ses cours; la maison qui protège la famille; ellemême souvent entourée et comme immergée dans d'autres maisons pour former un îlot irrégulier, desservi par une impasse; puis le quartier, constitué de quelques îlots; enfin la ville ceinte de murailles bien délimitées face à l'infini du désert (Salat, 2011: 64).

Le second correspond aux manières par lesquelles les différentes façons de s'approprier la ville et de faire partie de ses processus morphogènes s'enchaînent, pour donner forme à un système dynamique et hétérogène de gouvernance territoriale à échelles multiples, ce qui :

renvoie à l'idée de régime - moteur d'évolution du système urbain, c'està-dire à des caractéristiques variables telles que la puissance de contrôle du système, la capacité de mise en mouvement, la vitesse des cycles d'évolution, la capacité à créer de la cohésion et de la résilience (Declève, 2013: 14).

Il s'agirait donc d'une continuité qui touche les éléments du système urbain. Cette continuité urbaine, cependant, n'a rien à voir avec un ordre linéal ou déterministe qui tend vers l'homogénéisation et l'imposition d'une vision ou d'une version réductrice de la réalité; il s'agit plutôt d'une continuité rhizomique, comme je l'ai montré ailleurs (2013), pour faire référence à Gilles Deleuze et Félix Guattari. Celle-ci se nourrit de la diversité de situations urbaines et de la complexité de leurs relations, en permettant ainsi la création de points de connexion, de passages d'une dimension à une autre, c'est-à-dire d'une échelle à une autre. 
Les échelles spatiales ou morphologiques et les échelles de gestion et de gouvernance sont interconnectées et forment ensemble un système qui devrait fonctionner de manière solidaire. Cependant, ce système ne nie pas l'importance de la structure hiérarchique du système urbain, tant dans ses composants matériels qu'immatériels. Par contre, on doit nécessairement la repenser dans un contexte de réflexion élargi, prenant en compte le régime d'urbanité spécifique de la ville, qui puisse permettre de réorienter les approches vers de nouvelles hiérarchies non disjonctives. Dans ce contexte, l'organisation hiérarchique des systèmes urbains nécessaire au fonctionnement de la ville ne se réduit pas à la négation d'une échelle inférieure par l'imposition d'autres supérieures. À la fin des années 1960, Christopher Alexander avait déjà montré l'importance de cette vision complexe et intégratrice:

En bref, aucun pattern n'est une entité isolée. Chaque pattern peut exister dans le monde seulement dans la mesure où il est soutenu par d'autres patterns: des patterns plus grands dans lesquels il est incrusté, ceux de la même taille qui l'entourent, et des patterns plus petits qui sont incrustés en lui (1977: XIII).

Suivant ce cadre d'analyse, on doit nécessairement valoriser et amplifier l'incrémentation de degrés de continuité urbaine. Par ailleurs, on doit convoquer la notion de continuité urbaine dans les approches méthodologiques voulant décrire et analyser la morphologie des territoires et leurs processus de morphogenèse, comme dans les actions visant leur transformation. Étayer des démarches de recherche ou d'action sur une vision complexe conduit indéfectiblement à prendre conscience du caractère insaisissable de la vie urbaine et, surtout, des limitations des outils et des catégories de pensée en usage dans le domaine de l'urbanisme, qui se révèlent bien souvent incapables de rendre compte d'une réalité aussi complexe et souvent paradoxale.

Mais, au-delà des défis conceptuels et méthodologiques que la reconnaissance de la complexité impose aux démarches de recherche, c'est son implication dans les processus de décision, portés par l'action urbanistique publique visant à réguler et à transformer concrètement l'espace urbain, qui soulève le plus de questions. Il reste cependant à 
définir la manière dont les instances publiques, en s'appuyant sur cette reconnaissance, peuvent dépasser leurs conceptions et leurs méthodes traditionnelles d'action ancrées dans des paradigmes qui sont, pour la plupart, mal adaptés, voire parfois obsolètes et inopérants.

\section{Échelles spatiales et échelles de gouvernance. Détour par une ville moyenne}

Les changements dans les relations entre les villes et les territoires qui ont émergé, d'une manière plus évidente, à la fin du siècle passé, ont redéfini les interactions entre les échelles. Les centres urbains relativement importants, cherchant à se faire une place dans les circuits globaux, se sont inspirés de référents urbanistiques et de modèles urbains homogénéisés et de plus en plus déconnectés des réalités locales. En conséquence, on a largement relégué au second plan l'attention qu'on porte aux échelles inférieures, plus locales, plus quotidiennes, ainsi qu'à la mise en place d'éléments qui assurent les points de connexion et d'échanges interscalaires. Ce fait est particulièrement vérifié dans les centres urbains moyens, pour lesquels une incorporation effective dans les circuits globaux reste assez limitée, au moins dans les conditions qu'ils imposent.

L'exemple de Cochabamba est, à ce titre, particulièrement parlant. Située en Bolivie, Cochabamba est une ville moyenne latino-américaine comme n'importe quelle autre, mais qui, puisque c'est elle, n'est justement pas n'importe laquelle (l'appellation ville moyenne «américaine» pourrait entraîner de mauvaises interprétations $\left.{ }^{2}\right)$. Cette ville, qui compte 600 ooo habitants environ ${ }^{3}$, est la capitale de son département homonyme, un des neuf départements dont le territoire

2. L’hégémonie des États-Unis lui a donné le «droit» de s'approprier, en quelque sorte, le nom de tout un continent. Une ville «américaine» n'est pas seulement une ville des États-Unis, elle est, avant tout, une ville du continent américain. Donc, une ville moyenne d'Amérique du Sud est une ville moyenne américaine, elle ne doit pas se situer en Amérique du Nord pour l'être. Voilà un petit exemple de la puissance des grands centres, et de cette image du monde évoquée précédemment.

3. Selon les projections de l'Institut national de statistique (INE) de Bolivie, la ville de Cochabamba comptait, en 2010, 618376 habitants (www.ine.gov.bo). 
bolivien est composé. Les terres fertiles de ces vallées, situées à un peu plus de $1600 \mathrm{~m}$ d'altitude, ont joué un rôle très important dans l'histoire de la nation.

Que ce soit à cause de son potentiel agricole - même s'il est actuellement très diminué - ou de son climat favorable, la centralité géographique de cette ville à l'échelle du pays s'est vue accentuée par des mouvements migratoires qui ont donné forme à son contenu social, très divers et très caractéristique à la fois. Actuellement, Cochabamba est un carrefour commercial très important où la diversité sociale et l'économie informelle sont devenues déterminantes pour son développement et essentielles à son fonctionnement. La ville est chargée d'une histoire très riche en transformations, mais aussi en permanences; c'est un espace où ce qui se trouve généralement caché, profondément ancré dans les échelles les plus petites de la vie quotidienne, ou ce qui se développe sans vouloir attirer l'attention, joue un rôle de premier plan.

Au-delà de ces aspects qui font de Cochabamba un contexte intéressant dans cette analyse, cette ville constitue un prétexte pour un questionnement plus large portant sur les visions simplistes et homogénéisantes qui font de la figure de la métropole la synecdoque d'une constellation de villes petites et moyennes sur lesquelles elle soutient ses structures sociales, culturelles, et productives. En effet, on a largement sous-estimé le rôle des villes moyennes dans les dynamiques d'urbanisation et la structuration des territoires en Amérique latine, les recherches portant leur attention sur des espaces urbains considérés comme plus importants, notamment Sao Paulo, Buenos Aires, Lima ou Santiago. Bien évidemment, l'Amérique latine est beaucoup plus que ces grandes villes, elle est aussi Cochabamba et toutes les autres Cochabamba dispersées sur ce vaste continent. Malgré les fortes pressions que les grands processus de métropolisation exercent sur tout le continent, les villes moyennes ont su subsister. Elles ont fait preuve d'une étonnante capacité de résilience, même dans leur situation périphérique vis-à-vis des circuits globaux. L'intégration à ces derniers est pourtant considérée comme inévitable pour toute ville prétendant à se développer, de même que la métro- 
polisation - ou plutôt les processus qui en font la substance - est souvent présentée comme la seule voie possible de développement et, par extension, la seule version du monde urbain qui mérite d'être racontée. Dans le contexte latino-américain, beaucoup de villes moyennes ont récemment mis en œuvre des actions visant à se constituer en centres de territoires métropolitains ${ }^{4}$.

Dans cette ville moyenne en particulier, la situation que nous venons de décrire donne à voir différents phénomènes liés à la sousestimation des échelles inférieures évoquées précédemment. Nous pouvons souligner les tentatives de réguler l'espace urbain par l'action ponctuelle sans vision systémique, ce qui se traduit très souvent par la construction d'infrastructures à l'échelle prétendument métropolitaine, complètement déconnectées des conditions réelles du tissu urbain comme de son fonctionnement. En d'autres mots, nous pouvons noter de manière générale l'absence de coresponsabilité, en matière d'articulation entre échelles, dans les processus de production de la ville. Ainsi, s'intéresser aux systèmes de fonctionnement des villes intermédiaires, en s'éloignant des catégories métropolitaines d'évaluation et en privilégiant une ligne de pensée proche de celle que propose l'école territorialiste italienne, exposée dans Le projet local d'Alberto Magnaghi (2003), constitue une piste originale pour l'imagination d'autres types possibles de métropoles.

À Cochabamba en particulier, et en ce qui concerne les échelles spatiales, les actions que mène l'urbanisme institutionnel visant la structuration de l'espace urbain se sont limitées, la plupart du temps, à la production d'infrastructures ponctuelles de taille considérable. Ces interventions et leur présence imposante dans le paysage urbain donnent l'impression de répondre plus à un besoin de légitimation - notamment pour rappeler la présence d'une instance publique chargée d'organiser la ville - qu'à une véritable volonté d'améliorer les conditions de fonctionnement du système urbain.

4. Dans le cas spécifique de Cochabamba, ce processus, qui depuis plusieurs décennies a changé des termes comme "conurbation» par celui de "zone métropolitaine», n’a été réellement institutionnalisé que récemment. 
Les exemples qui peuvent illustrer cette situation sont nombreux. Comme pour n'importe quelle ville procédant d'une gestion fortement centralisée, la circulation dans le centre-ville de Cochabamba et ses environs devient chaque année de plus en plus problématique. Le fonctionnement chaotique du système de transport public et l'omniprésence de la voiture n'ont pas seulement généré de graves problèmes de congestion. Ils ont aussi presque éliminé du paysage les autres moyens de transport, comme le vélo. Finalement, en exerçant une pression étouffante sur les espaces de la ville, ils ont largement contribué à la dégradation des espaces destinés aux piétons. Face à tous ces problèmes, qui ne se réduisent évidemment pas à une question d'organisation mécanique des flux, les réponses que l'urbanisme officiel a apportées se sont limitées à la construction d'importants échangeurs aux portes de la ville. Ces interventions parfois impressionnantes sont totalement déconnectées du tissu environnant et génèrent des fractures presque infranchissables pour les transports non motorisés. Sans aucune réflexion urbanistique systémique, ces artefacts s'avèrent incapables d'apporter des solutions à la congestion routière dans l'échelle supérieure du tissu viaire et se constituent en barrières importantes pour les fonctionnements urbains aux échelles inférieures.

À la fin des années 1990, les autorités municipales, bien conscientes de la perte progressive du vélo, ont lancé un projet qui utilisait le cours d'un vieux canal d'irrigation pour construire une piste cyclable de plus de $8 \mathrm{~km}$ connectant l'est et l'ouest du nord de la ville. Au début, le projet a été bien accueilli. Cependant, étant donné que son tracé évite le centre-ville et ses environs et qu'il ne connecte pas réellement les principales zones d'activités, il fut dès le départ voué à l'échec. Aujourd'hui la ciclovia de la ville n'est utilisée que très rarement, et peu de personnes l'utilisent dans leurs trajets quotidiens. C'est donc un autre exemple d'une intervention ayant beaucoup de potentiel, mais dont la portée urbanistique fut très limitée. Le vélo était vu comme un simple outil de loisir et non comme un moyen de transport à part entière. Ce projet, très important à l'échelle de la ville, n’a pas réussi à mettre en relation les petites échelles de la vie quotidienne (pas même 
des quartiers qu'il traverse) avec l'ensemble de la ville ou, au moins, avec une bonne partie d'elle. Au fil du temps, il est devenu absolument anecdotique dans l'ensemble du tissu urbain.

Pour essayer de mieux saisir ces phénomènes, il est nécessaire de comprendre que la ville est produite par de complexes jeux d'alliances et de conflits entre acteurs qui construisent plusieurs villes en même temps. Chacune d'elles recompose la structuration même de la ville en assignant des fonctions et des valeurs très diverses aux différentes échelles qui la composent. L'analyse des dynamiques que produit l'espace urbain de cette ville en particulier permet de comprendre comment les systèmes formels et informels travaillent ensemble.

En même temps que ces deux systèmes s'opposent, chacun est tributaire de l'autre. Les instances régulatrices du système de production formel de la ville cherchent à contrôler l'expansion urbaine et à rationaliser l'occupation du sol, en laissant une grande marge d'action aux instances informelles pour affirmer leurs rôles dans la définition des modalités dans lesquelles ces processus se développent. Face à l'agrandissement du territoire métropolitain par l'incorporation de nouvelles zones urbanisées, les systèmes informels se chargent de connecter ces nouvelles zones avec la ville, et cela bien avant leur reconnaissance officielle. Comme on l'a souligné ci-dessus, alors que la réponse institutionnelle pour combattre la congestion se concentre sur le développement des infrastructures, le fonctionnement et la gestion des flux de transport sont entre les mains d'agents privés et de circuits informels. Si l'action officielle veut avant tout équiper certains des grands espaces publics pour les transformer en points de référence à l'échelle de la ville, l'action informelle se charge d'animer les espaces publics à l'échelle du quartier.

Au milieu de ces dynamiques complémentaires, l'acteur/habitant construit sa partie de ville en prenant ce que les deux systèmes lui offrent, tout en apportant de nouveaux éléments venus des confrontations entre différentes formes d'habiter exprimées à l'échelle du quartier. Ce sont les résultats de ces confrontations qui déterminent le renforcement d'un des deux systèmes au détriment de l'autre, selon 
les cas. C'est ainsi que dans cette ville, comme dans toutes les autres, l'habiter fait partie à part entière des acteurs producteurs de l'urbain. Ainsi, de l'aire métropolitaine à l'habitant qui la parcourt tous les jours, il existe une diversité d'échelles d'action, de décision et de gouvernance, qui renvoient à des mécaniques urbaines et urbanistiques très diverses. Chacune des échelles redéfinit la notion de la ville en tant qu'œuvre collective, participant finalement à la constitution de ce régime d'urbanité particulier.

La perte progressive de l'échelle du quartier comme élément articulateur entre l'espace résidentiel et la ville (pratiquée, représentée et appropriée) ne renvoie pas seulement à des dynamiques spatiotemporelles propres aux villes contemporaines : c'est aussi un phénomène qui se développe progressivement à l'intérieur du quartier, tout au long de sa vie. Dans le contexte spécifique de Cochabamba, plus un quartier a de bonnes conditions d'habitabilité, plus les niveaux de participation de ses habitants dans les processus de décision et de gestion sont faibles. De manière schématique, on observe trois figures élémentaires des quartiers en tant qu'espaces d'interaction et de sociabilité (même s'il faut émettre quelques nuances):

- Les quartiers en formation se développent généralement collés à la ville, hors de ses limites administratives et sur des terrains qu'on destine généralement à des activités agricoles ou à la protection environnementale. Dans ces quartiers, la cohésion et une certaine homogénéisation sociales sont absolument nécessaires. La solidarité entre habitants leur permet de faire face collectivement aux difficultés causées par l'absence de services de base et de consolider une masse critique suffisante pour entamer des négociations avec les autorités. Ces tractations peuvent mener à la reconnaissance officielle du quartier en tant qu'Organisation territoriale de base $(\mathrm{OTB})^{5}$, qui représente la

5. À partir de la Loi de participation populaire de 1994, les nouveaux quartiers ou comités de voisinage ont la possibilité, après un processus administratif et technique, d'être reconnus en tant qu'OTB, ce qui veut dire qu'ils peuvent accéder de manière 
première échelle de l'administration du territoire gérée directement par les habitants.

- Les quartiers consolidés englobent la plupart des quartiers résidentiels de la ville. La diversité des situations qu'elle réunit est donc très vaste. Néanmoins, comme nous l'avons évoqué précédemment, l'amélioration des conditions d'habitabilité dilue la participation des habitants dans les démarches collectives (il n'y a plus de raisons pour se mobiliser). Parmi les multiples causes de ce phénomène qu’on peut évoquer, les caractéristiques de l'espace physique, à savoir les degrés de continuité entre les articulations des éléments du tissu urbain, apparaissent centrales. Dans ces quartiers, il s'observe une division absolue entre les espaces privés et les espaces publics (les habitants ne s'approprient plus la rue, séparée de la parcelle résidentielle par un mur, les espaces verts sont atomisés et n'arrivent pas à constituer un système, etc.).

- Les quartiers fermés, très divers entre eux, sont généralement l'expression de l'individualisation des modes de vie et de la quête d'homogénéisation dans l'espace résidentiel comme dans les relations sociales. Ces quartiers produisent des discontinuités dans l'espace physique, puisqu'ils sont fermés et donc complètement imperméables, et dans l'espace social, car leurs habitants n'entretiennent pas ou très peu de relations avec leurs voisins.

Voilà trois façons de mettre en relation l'espace résidentiel privé avec l'espace collectif de la ville; trois fonctionnements différents de l'échelle résidentielle et de l'échelle du quartier et trois situations de gouvernance distinctes qui appellent à remettre en question les manières dont l'action urbanistique publique cherche, ou non, à les articuler à l'échelle de la ville.

directe à des fonds de l'État, prendre des décisions concernant les projets à voir en priorité et veiller à leur mise en œuvre. 


\section{Vers un urbanisme de la continuité}

Le manque de points d'articulation entre les échelles, qu'elles soient spatiales ou sociales, c'est-à-dire de gouvernance, produit des pathologies dans le système urbain qui, loin de produire des effets isolés, ont une répercussion véritablement multiscalaire. Le contexte de cette ville moyenne, où les images de la métropole se présentent comme la panacée du développement urbain, révèle à la fois de nombreux problèmes que génère l'institutionnalisation d'une vision simpliste et fragmentaire, mais aussi quelques pistes de réflexion.

Face aux dysfonctionnements, la vision simpliste avec laquelle l'action urbanistique publique agit sur les dynamiques urbaines fait souvent appel à la même logique, à savoir la mise en place ou le renforcement d'une échelle hiérarchique supérieure. Du côté de la gouvernance, la faible implication des instances officielles dans les dynamiques quotidiennes des habitants réduit radicalement les possibilités d'entamer un travail plus solidaire et mieux coordonné.

Par ailleurs, tant du côté des échelles spatiales que des échelles de gouvernance du quartier, les enjeux du contact direct avec la vie quotidienne de la population restent une piste centrale et très féconde pour repenser les articulations entre les composants du système urbain: «l'essentiel des débats se fait toutefois le plus souvent à une échelle, celle des quartiers» (Hubert, Lewis et Reynaud, 2014: 397). Cependant, on doit aussi considérer l'échelle du quartier comme un système en soi et non pas comme la simple juxtaposition des espaces de résidence:

En bref, les quartiers qui fonctionnent ne sont pas des entités séparées les unes des autres. Au contraire, sur le plan matériel, social, et économique, ils sont étroitement liés les uns aux autres, à petite échelle certes, mais à la manière des fibres qui constituent une corde (Jacobs, 2012: 114).

Par ces considérations, penser l'action urbanistique institutionnelle comme un des producteurs de la ville parmi beaucoup d'autres, c'est-à-dire comme une partie du système, oblige à l'extraire des sphères technocratiques très restreintes avec lesquelles elle est com- 
munément associée. Chaque force qui intervient dans la production de la ville, que ce soit l'urbanisme (acteur urbanisant présumé) ou l'habiter (acteur urbanisé présumé), avec ses potentialités et ses limites, apporte un élément de plus à ce processus complexe. Reconnaître les capacités de chaque participant, ainsi que leurs échelles d'action, et s'approcher de la compréhension de ces interactions avec les autres, c'est faire un pas important vers la production collective d'une ville, qui non seulement trouve dans la diversité de ses éléments les fondements de son existence, mais aussi le moteur de son développement. Cela passe par ailleurs par la redéfinition de l'action urbaine institutionnelle comme un outil en plus pour l'action collective sur l'espace, un instrument qui favorise les articulations complexes et, par conséquent, la production de la continuité urbaine. Un outil promouvant l'appropriation des processus de morphogenèse de l'espace par les habitants et les organisations sociales permettrait de ne pas les considérer simplement comme des utilisateurs des objets et des systèmes pensés, mais aussi comme des acteurs constructeurs de la ville. Ainsi, l'action urbaine institutionnelle doit aussi être une pratique de l'interstice, du contact avec les échelles plus petites de la vie quotidienne. Elle ne doit pas seulement être perçue comme une sphère de médiation des conflits ni s'apparenter à un outil de régulation absolu, mais plutôt devenir un catalyseur d'interactions multidimensionnelles et multi-échelles, c'est-à-dire un producteur de continuité. 



\title{
CHAPITRE 6
}

\section{De la planification stratégique au droit à la ville}

\author{
Issa Sory
}

\begin{abstract}
À la faveur d'un mouvement de décentralisation, de libéralisation et la démocratisation, le Burkina Faso a entrepris, ces dernières années, une série de réformes institutionnelles et opérationnelles. Celles-ci sont perceptibles surtout dans le domaine de la production et de la gestion d'espaces urbains.

À partir du début des années 1990, différentes réformes - aux objectifs convergents - se sont télescopées au Burkina Faso. Après quatre années de régime révolutionnaire (1983-1987), les institutions financières internationales (IFI) considèrent le Burkina Faso comme un pays à la périphérie des politiques néolibérales, en cours dans la majorité des pays africains francophones. Ces politiques ont été mises en œuvre dans le cadre de l'application des Plans d'ajustement structurel (PAS) à la fin des années 1970. Dans une logique d'harmonisation (par le rattrapage), les IFI ont imposé les PAS au Burkina Faso dès 1991. Cette option néolibérale s'est ressentie dans la version de 1991 de la Réorganisation agraire et foncière (RAF), ainsi que sur les axes d'intervention des deuxième et troisième projets de développement urbain, par ailleurs financés par les IFI. Un processus de décentralisation est aussi enclenché dans la foulée à partir de 1993. Les
\end{abstract}


premières élections municipales sont organisées en 1995 dans quelques communes, et sur toute l'étendue du territoire à partir de 2006.

Le contexte néolibéral et de décentralisation implique la remise en cause de la planification urbaine classique, tout en offrant un cadre favorable à l'adoption de la planification territoriale stratégique. En rappel, le concept de néolibéralisme, développé après la Seconde Guerre mondiale, désigne cette vision politique et idéologique ayant pour but, entre autres, l'érection de l'individualisme en valeur cardinale de la société et la promotion de l'économie de marché. En milieu urbain, il s'agit surtout de la délégitimation des services publics au profit du secteur privé. Ainsi, les partenariats public-privé, qui ont recours aux grandes entreprises (nationales ou internationales) ou aux petits opérateurs privés, constituent d'autres formes de déclinaison du néolibéralisme. La notion de démocratisation, quant à elle, accompagne explicitement le processus de décentralisation par la création présupposée d'espaces de liberté permettant, du moins théoriquement, de favoriser la participation politique et les revendications citoyennes.

Ouagadougou, capitale du Burkina Faso, offre un terrain propice d'observation et d'analyse des différentes réformes en général. Pour cela, la prise en considération du double jeu d'échelles - entre le local et le global - permet de mieux cerner les pratiques des acteurs locaux.

\section{Des produits de la décentralisation}

Confronter la planification territoriale stratégique au droit à la ville constitue un exercice complexe. En effet, issus de deux idéologies radicalement opposées - respectivement le libéralisme et le marxisme -, ces concepts suscitent des confusions dans leur application. La planification territoriale stratégique - démarche d'aménagement qui tend à s'imposer globalement depuis les années 1990 - apparaît comme une forme plus aboutie dans le domaine de la coordination des acteurs urbains. Elle semble aussi mieux adaptée aux contextes institutionnels actuels des villes africaines francophones. La délégitimation de la 
planification urbaine centralisatrice ou planification urbaine classique est passée notamment par l'émergence de "gouvernements locaux» (Josse et Vauquelin, 2011). Ces nouveaux gouvernements sont promus par la décentralisation. Cette nouvelle démarche de planification met l'accent sur la participation des acteurs de la production de l'espace urbain (citadins, acteurs privés, autorités municipales et étatiques) aux projets d'aménagement. Elle participe ainsi à la réappropriation de l'espace urbain par les citoyens. Grâce à la participation des citadins, la démarche de planification territoriale stratégique peut donc s'apparenter, du moins en théorie, à celle du programme politique de réforme urbaine pour le droit à la ville (Lefebvre, 1967).

Au Burkina Faso, l'idée de la participation des citadins à la production de l'espace urbain est au cœur des réformes décentralisatrices. Le transfert de la compétence de gestion foncière aux collectivités locales vise, en effet, une meilleure prise en compte des aspirations des citoyens. Au cours de la même période, les prescriptions du troisième projet de développement urbain ont remis en cause implicitement la planification urbaine classique.

En effet, ce projet comprenait plusieurs composantes dont celle concernant le renforcement des capacités des acteurs institutionnels. Cette dernière intégrait trois volets : 1) la mobilisation des ressources, surtout fiscales; 2) la gestion du foncier urbain; 3) le renforcement de la participation communautaire, de la formation, de l'assistance technique et de l'équipement de la ville. Le volet «mobilisation des ressources fiscales» encourageait le renforcement du système urbain d'information, l'application effective du principe du pollueur payeur par la participation des populations urbaines à la couverture des charges induites par l'amélioration des conditions de vie, ainsi que l'intégration du plus grand nombre de citadins dans le système fiscal. Ce volet se focalisait donc essentiellement sur la mise en place d'un mécanisme de recouvrement efficace des taxes de résidence qui, selon le projet, était indispensable à l'accélération du développement urbain. Suivant la même logique, le volet concernant la gestion du foncier urbain mettait l'accent sur l'amélioration de l'information foncière et 
la réorganisation des Directions régionales de l'urbanisme et de la topographie (DRUT). Il s'agissait notamment de constituer des bases de données fiables sur l'occupation du sol ayant pour identifiant unique l'adresse de la parcelle. L'importance de ces bases nécessitait, selon le troisième projet de développement urbain, qu'elles soient régulièrement mises à jour pour cerner la dynamique de la production de l'espace urbain en vue de permettre une meilleure planification de la ville. La Banque mondiale considérait ainsi les outils classiques de planification urbaine - schéma directeur d'aménagement urbain (SDAU) et plan d'occupation des sols (POS) - comme peu adaptés aux nouveaux enjeux de développement urbain du Burkina Faso et à ses réalités locales. Du fait de leur durée d'élaboration, relativement longue, ces outils, une fois finalisés, n'étaient plus en phase avec les dynamiques urbaines à l'œuvre, y compris avec le rythme d'étalement de la ville concernée. Ainsi on a suggéré de passer à l'élaboration de plans de référence, plus simples et plus flexibles, concevables dans un temps court et facilement réaménageables. Pour l'actualisation des plans, on a prévu des méthodes incluant l'utilisation d'images satellites et d'enquêtes périodiques.

Dans les prescriptions des institutions financières internationales, il s'agissait de faire amorcer, par le pays, une transition dans les approches de planification: passer de la planification traditionnelle et classique - avec des méthodes strictes de fabrication de la ville - à la planification territoriale stratégique, qui ne définit que les grandes orientations et les grands axes du développement urbain. Pour ces institutions, cette nouvelle méthode de planification pouvait permettre de corriger les insuffisances de la méthode antérieure. Elle avait aussi l'avantage de répondre aux impératifs néolibéraux des bailleurs de fonds, qui consistent à ficher et taxer les citadins et à impliquer les acteurs privés dans la production, le contrôle, l'application et la modification des outils de la planification urbaine.

Dans ce contexte, on a créé de nombreuses associations de résidents ou de crise dans les différentes localités du Burkina Faso, mais plus particulièrement à Ouagadougou. Elles sont par ailleurs plus 
présentes dans les quartiers informels. L'objectif commun à ces associations est de participer aux opérations d'aménagement urbain. Elles revendiquent l'attribution des parcelles ou des lots aux résidents réels ${ }^{1}$. Paradoxalement, contre les objectifs des citadins qui correspondent aux attentes de la démarche de planification territoriale stratégique (la participation citoyenne), les autorités étatiques et municipales érigent des barrières qui contribuent à l'exclusion de ces associations des instances de l'aménagement urbain. Les revendications pour leur participation aux comités d'attribution des parcelles n'ont jamais été satisfaites. Finalement, on a supprimé la possibilité de participation de ces regroupements associatifs par la nouvelle loi portant sur la réorganisation agraire et foncière ${ }^{2}$. Cette dernière ne prévoit pas de possibilité de participation des citoyens aux opérations d'aménagement urbain. Désormais, comme le stipule l'article 159 de la loi : «la cession des terres du domaine privé immobilier non affecté des collectivités territoriales est faite par l'administration locale, par adjudication, par tirage au sort ou de gré à gré, après instruction des dossiers de demande par les services compétents».

\section{L'importation de concepts}

L'analyse des concepts de planification territoriale stratégique et du droit à la ville, dans le contexte ouagalais, révèle plusieurs difficultés essentiellement liées à la particularité du contexte. On a développé ces concepts dans un environnement institutionnel tout à fait différent de celui de Ouagadougou.

Le modèle de planification territoriale stratégique observable au Burkina Faso, et à Ouagadougou en particulier, est celui importé depuis la France; tous les outils d'aménagement urbain sont des calques de ceux de l'ancienne métropole. Cette nouvelle forme de planification n'est cependant pas explicite lors des opérations d'aménagement au

1. Sont considérés comme résidents réels les citadins habitant effectivement des quartiers informels et ayant été recensés par les services en charge du lotissement.

2. Il s'agit de la loi n 034-2012/AN du 2 juillet 2012. 
Burkina Faso. Il n'est fait référence à la planification territoriale stratégique dans aucun outil d'aménagement urbain. Par conséquent, sans une approche systémique, il est impossible de saisir les mutations opérées dans les outils d'aménagement du Burkina Faso depuis les années 1990. Au-delà de cette difficulté, le processus de transfert d'outils ou de modèles de planification pose des problèmes d'échelle et de gouvernance urbaine. En effet, les échelles géographiques d'application de ces outils sont souvent différentes de celles de la France. Il en est de même pour la temporalité de leur application. Aussi, l'exercice municipal en France - ne seraitce que la relative autonomie des pouvoirs locaux vis-à-vis du pouvoir central - est très différent de celui au Burkina Faso. Enfin, il est courant de constater au Burkina Faso l'inexistence d'outils de planification (c'est le cas des petites et des moyennes villes jusqu'à une date récente) ou leur faible application (cas de Ouagadougou). Tous ces facteurs exigent donc une certaine prudence lorsqu'il s'agit d'appliquer des concepts et des outils exogènes.

Mettre à l'épreuve l'évolution des logiques de planification avec la démocratisation et l'amélioration attendue du droit à la ville est tout aussi problématique. Concept formalisé par Henri Lefebvre à la veille de Mai 1968, la notion de droit à la ville est devenue, particulièrement au cours des années 2000, un concept que mobilise l'ONU, ainsi que certains États ou municipalités, pour promouvoir la participation citoyenne, l'émergence de nouvelles formes de mobilisation politique aux échelles locales ou encore l'implication des citadins dans les processus d'aménagement. Bien entendu, la portée du concept a largement changé de nature entre l'approche théorique que propose Lefebvre et la mobilisation du terme par des organisations institutionnelles ou civiles. Cependant, au même titre que la bonne gouvernance, le droit à la ville est devenu peu à peu un corollaire de l'amélioration de la participation citoyenne, qui accompagne théoriquement les processus de décentralisation et de réorganisation des logiques d'aménagement évoquées plus haut.

La mobilisation de cette expression dans le contexte ouagalais doit donc faire l'objet d'une grande prudence. Si l'on s'en tient à la 
proposition de Lefebvre, le niveau d'urbanisation a certainement guidé l'accent mis sur la ville, perçue comme le creuset d'une possible révolution sociale. Ce contexte contraste totalement avec celui du Burkina Faso, où environ $80 \%$ de la population réside en milieu rural. Ensuite, le concept de droit à la ville se voulait une réflexion évoquant notamment les répercussions du capitalisme et de l'économie de marché sur l'espace politique de la cité. Si on peut considérer la France comme un pays capitaliste développé, le Burkina Faso constitue un pays périphérique. Ce pays souffre à la fois des effets du système capitaliste et des conséquences de son non-développement. De ce fait, si le concept de droit à la ville est mobilisé dans le contexte ouagalais, il ne peut évoquer tout à fait la même chose. Le droit à la ville évoque de manière croissante la participation citoyenne et la démocratisation, notamment dans la sphère du développement. Il semble intéressant de le mettre en perspective avec l'évolution des logiques de planification décrites précédemment. Est-ce possible d'importer, sur le plan scientifique, les concepts de planification territoriale stratégique et du droit à la ville à Ouagadougou? Quelles sont les précautions à observer dans l'utilisation de ces concepts en milieu urbain burkinabè, et plus particulièrement à Ouagadougou?

\section{L’importance des échelles géographiques}

La convocation des concepts de planification territoriale stratégique et du droit à la ville à Ouagadougou implique une posture itérative - des allers-retours entre le local, le national et le global - prenant appui sur les pratiques des acteurs à l'échelle locale. Il s'agit de comprendre le contenu théorique de ces concepts en vue de saisir leurs déclinaisons à Ouagadougou. Les pratiques des acteurs de la production de l'espace urbain et de la gestion urbaine (à l'échelle municipale ou de quartier) servent ainsi de guides pour l'analyse. De ce fait, le but de la démarche est plus d'enrichir les concepts à partir d'autres expériences que de procéder à leur plâtrage dans un contexte différent. 
Dans cet exercice, la prise en compte de la nature du régime politique burkinabè est indispensable pour mieux appréhender le fonctionnement des villes. En effet, le paradoxe concernant la participation citoyenne aux opérations d'aménagement urbain est caractéristique du fonctionnement du régime semi-autoritaire ${ }^{3}$. La création d'un environnement institutionnel favorable à cette participation est accompagnée par des mécanismes ayant pour objectif sa confiscation. Ces contradictions se délocalisent de l'échelle nationale jusque dans tous les segments de la gestion urbaine.

Dans le domaine de la gestion des déchets par exemple, les autorités municipales ouagalaises ont entrepris, depuis les années 2000, des réformes visant à responsabiliser les précollecteurs sur des territoires urbains délimités à cet effet. Ouagadougou a été ainsi subdivisée en douze territoires de précollecte. On a ensuite concédé ces territoires aux groupements d'intérêt économique (GIE) - des regroupements d'associations ayant des objectifs lucratifs - et aux petites et moyennes entreprises (PME) par l'intermédiaire d'un appel d'offres. Ces concessionnaires de la filière ont obtenu de la part des autorités la garantie d'être les seuls acteurs sur leurs territoires respectifs. Selon le Schéma directeur de gestion des déchets (SDGD) ${ }^{4}$, outil technique de référence de la réforme, cette réorganisation de la filière a pour objectif de responsabiliser ces acteurs. La multitude de précollecteurs sur le territoire urbain devait donc être interdite pour ne laisser que les nouveaux. La contribution de la police municipale était attendue afin d'asseoir la réforme. Elle avait surtout la responsabilité d'appréhender les précollecteurs non concessionnaires, c'està-dire les acteurs informels. Paradoxalement, après quelques années de mise en œuvre de la réforme, la filière des déchets de Ouagadougou connaît la persistance de ces précollecteurs informels. L'une des principales raisons est qu'ils sont soutenus par les élus locaux. Ainsi,

3. Pour plus d'informations sur le régime politique semi-autoritaire, voir notamment: Laurent, 2000; Ottaway, 2003; Hilgers et Mazzocchetti, 2006.

4. L'élaboration du SDGD et sa mise en œuvre ont été financées par la Banque mondiale dans le cadre du troisième projet de développement urbain. 
ceux qui ont adopté le SDGD et devaient, avec les équipes municipales, œuvrer à son application sont les artisans de la désorganisation de la filière. Les conseillers municipaux participent à l'organisation, à la formalisation et au maintien des précollecteurs informels à Ouagadougou. Ces conseillers aident, en effet, les acteurs informels de la précollecte des déchets à s'organiser en associations par leur participation active à la rédaction des textes de l'organisation et le suivi du dossier de légalisation.

Ces différents paradoxes ne peuvent être compris que par la compréhension des assises politiques du régime semi-autoritaire. Celui-ci se caractérise par la présence de pratiques démocratiques et autoritaires. Ce type de régime œuvre à la création d'espaces de liberté permettant la libre expression et l'existence d'une opposition. Dans le même temps, par des dispositifs informels, il travaille à aliéner le champ d'expression des libertés, tout en rendant l'alternance impossible. Dans ce double jeu, ce régime assoit et consolide le pouvoir; un pouvoir désormais reconnu à la fois à l'échelle internationale (résultat de son aspect démocratique) et locale (du fait de son caractère autoritaire). Son apparence démocratique (officielle) est orientée vers les bailleurs de fonds internationaux, tandis que ses pratiques autoritaires (officieuses) garantissent sa durabilité. Le fonctionnement du régime politique semi-autoritaire laisse entrevoir que la réalité locale se saisit par la transcription des pratiques informelles guidées par les discours officieux. Ainsi, les discours officiels ne s'apparentent qu'à de véritables trompe-l'œil.

L'exemple de la désorganisation de la filière des déchets dans le soutien des associations enseigne ainsi que l'adoption du SDGD participe d'une stratégie d'acceptation du modèle proposé par la Banque mondiale tandis que les relations, apparemment paradoxales, entre les conseillers municipaux contribuent à entretenir l'assise du pouvoir municipal et étatique. En effet, le soutien que les conseillers apportent aux précollecteurs - par les actions ou les influences politiques permettant leur reconnaissance officielle - est partie intégrante de leur stratégie du maintien de leur base électorale. 
Concernant la confiscation de la participation citoyenne aux instances de gestion de la ville, le schéma fonctionnel du double jeu du régime semi-autoritaire peut constituer une grille d'analyse efficace. Les autorités municipales et étatiques ont emprunté les discours politiques en faveur de la participation du modèle de gouvernance que proposent les organismes internationaux. Ces discours font partie de la légitimation internationale des institutions nationales et communales. Par contre, vu les enjeux politiques et économiques que couvent les opérations d'aménagement urbain, l'effectivité de cette participation peut compromettre les assises du régime. Par exemple, la participation des responsables d'association aux instances de lotissement pourrait réduire les chances de détournement de parcelles, l'un des principaux supports d'accumulation du capital économique et social des élus locaux.

À Ouagadougou, les projets de développement urbain des IFI, le contexte de démocratisation et de décentralisation et le passage à la planification de territoire stratégique ont suscité l'éclosion d'un contexte de participation à la gestion urbaine. Cet environnement institutionnel de participation sert ensuite à justifier les revendications des habitants des quartiers informels pour le droit à la ville. Cependant, la compréhension des jeux des acteurs étatiques et municipaux - liés à la confiscation de cette participation - nécessite le décryptage de la nature du régime politique au Burkina Faso. Dans le cas de Ouagadougou, le mode de fonctionnement du régime semiautoritaire implique, entre autres, l'analyse du décalage entre les discours et la pratique, ainsi que la prise en compte des différentes échelles géographiques de validation des projets urbains. 


\title{
CHAPITRE 7
}

\section{Mobilité, échelles et chemins}

\author{
Bianca De Marchi Moyano
}

L'émergence des technologies des transports et l'augmentation des flux de populations et de marchandises ont mis au centre des débats la problématique de la mobilité. En effet, comme Montulet et Kaufman le soulignent, "nous sommes en train de vivre une phase de transition entre un monde organisé autour de territoires délimités et un monde réticulaire caractérisé par une fluidité spatiale » (2004: 11). Cette mutation, qui transforme les dynamiques spatiotemporelles des sociétés, intensifie les relations et les tensions entre les échelles sociospatiales. En conséquence, les pratiques et les expériences de proximité et de distance sont sans cesse modifiées et se doivent d'être systématiquement remises en question.

Objet de structuration de l'espace, le chemin sera ici mobilisé et présenté comme un outil théorique, qui renvoie notamment aux différents modes de transport terrestre utilisés dans l'histoire et aux diverses manières de cheminer des sociétés. Le chemin est bien plus qu'une ligne connectant deux nœuds: c'est un objet qui permet l'analyse d'expériences particulières, de rapports sociaux et de modes de transport divers dans l'espace. La pratique du chemin et sa représentation font, en ce sens, partie intégrante de la production de l'espace.

En s'appuyant sur plusieurs exemples de projets de construction de chemins dans le département de Cochabamba en Bolivie, cette 
contribution considère le chemin à la fois comme un objet technique qui permet la communication entre les lieux, comme un scénario social que différents acteurs ont adopté et révélé dans leurs rapports sociaux et leurs discours, et enfin comme une clé de lecture pour aborder le débat sur les frontières territoriales et leur porosité dans les dynamiques de mobilité.

Les exemples montrent deux logiques de compréhension du territoire. La première est politico-administrative, ancrée dans les territoires délimités par des frontières. La seconde se rattache aux flux et aux réseaux de mobilité. Elles ne sont pas simplement opposées, mais entretiennent un rapport complexe. Les acteurs fabriquent des chemins pour élargir leur capacité à mobiliser des flux sur des territoires circonscrits par des limites plus ou moins marquées. Dans le même temps, ces frontières vont agir comme des lignes de contrôle de la mobilité et favoriser un certain type de flux. Ces rapports entre frontières et mobilités seront analysés par la prise en considération de différentes échelles d'action d'acteurs variés.

\section{Le chemin et ses échelles de la mobilité}

On considère généralement les chemins comme des objets permettant d'assurer la communication entre deux lieux. Ces lieux connectés contribuent, de manière générale, à donner du sens à la voie tout en caractérisant sa dynamique sociale. Par exemple, les territoires urbains, en cherchant à être reliés par de nouveaux projets routiers, interviennent en fonction de leurs intérêts, de leur position et des rapports de force pour définir le tracé, afin de contrôler et de profiter des nouveaux flux.

Cependant, les lieux d'origine, de destination et ceux traversés ne participent pas en tant que tels à l'énonciation et à l'élaboration de projets de développement territorial. Ceux-ci sont au contraire le résultat de stratégies, de discours et de représentations d'acteurs, et donc des logiques sociales qui conditionnent tant leurs intérêts que les rapports qu'ils entretiennent entre eux. Au sujet des enjeux de 
mobilité, les acteurs les plus concernés sont tant les opérateurs étatiques, privés et commerciaux, que les usagers.

Une observation diachronique permet de montrer que l'évolution de l'étalement urbain est largement déterminée par des corridors structurants et par l'apparition des nouveaux nœuds secondaires qui, en retour, participent à la croissance de la ville. La pression développée par ces nouveaux flux stimule la construction de l'espace habitable et productif. Cela est encore plus évident lorsque la mobilité automobile est dominante et que les possibilités d'expansion de l'espace parcouru par les individus sont grandes. Néanmoins, les dynamiques produites par les voies automobiles ne doivent pas occulter les transformations engendrées par les autres modes de transport.

Dans ce cadre d'analyse, les échelles sociospatiales prennent une importance particulière. D'une part, il existe des caractéristiques de structuration des réseaux viaires qui sont normalement déterminées par l'État dans chaque pays pour organiser les différents types de transports, ainsi que les routes et les chemins capables de les supporter. Cette approche met en évidence la capacité planificatrice et gestionnaire des acteurs officiels dont l'objectif est de maîtriser et de garantir leur contrôle aux différentes échelles territoriales.

Cependant, il existe aussi d'autres liens entre les dynamiques de circulation de personnes, de biens et d'informations (comme conséquence de la mondialisation). Ces liens modifient les dynamiques de la mobilité et interviennent sur son développement. Par ailleurs, la dynamique des acteurs (usagers quotidiens et décideurs intermédiaires $^{1}$ ) renvoie aussi à leur attachement à des points de repère proches ou distants, locaux ou globaux. Ainsi, les échelles hiérarchisées et structurées dans la planification officielle se trouvent débordées ou remises en cause. Il devient alors primordial de considérer cette variété et cette superposition d'échelles réticulaires pour cerner les tensions et les conflits qui émergent à l'endroit de leurs articulations.

1. Comme les représentants territoriaux, les élus et les techniciens des départements et des municipalités, etc. 


\section{Le chemin et les politiques territoriales nationales}

Le cas bolivien est intéressant pour étudier les politiques publiques territoriales, notamment celles du département de Cochabamba, où on a contrarié et arrêté deux projets routiers à la suite de conflits sociaux. Cochabamba est un territoire situé sur différentes régions écologiques, entre les systèmes de la cordillère des Andes et de la plaine amazonienne. C'est également un espace possédant une population très urbanisée. Son aire métropolitaine ${ }^{2}$ est peuplée de 1,2 million d'habitants environ établis sur une zone de vallées andines. Ils représentent $60 \%$ de la population départementale.

Les exemples de projets routiers s'avèrent particulièrement propices à l'étude du rôle de l'organisation plurinationale actuelle de l'État bolivien dans l'aménagement et la gestion du territoire. À ce stade de l'analyse, il est nécessaire de rappeler que la décentralisation (commencée dès les années 1980) est effective en Bolivie. Le pays est administrativement organisé en 9 départements et 327 municipalités. Ces municipalités constituent les territoires d'exercice de gouvernements locaux. Le département de Cochabamba est ainsi composé de 47 municipalités. Il existe par ailleurs des territoires administratifs spéciaux, comme les terres communautaires d'origine (TCO) destinées à la propriété collective des peuples indigènes, ou encore les parcs nationaux qui assurent la protection du patrimoine biologique.

En 2005, le premier président indigène de la Bolivie (mais aussi de l'Amérique du Sud) est élu. Evo Morales met au cœur de sa politique la fortification et la modernisation de l'État dans le domaine de la gestion des ressources naturelles et de l'organisation du territoire. Ainsi, la structuration du territoire à partir des infrastructures routières constitue une des priorités de son programme de développement. Dans la foulée et à partir de 2009, une nouvelle constitution est appliquée. La Bolivie devient donc un État plurinational où les rapports entre l'État et la nation se complexifient.

2. La ville de Cochabamba est considérée comme une "région métropolitaine» bolivienne. Cela ne signifie pas qu'elle est une grande "métropole», mais elle peut développer des outils de planification et d'aménagement correspondant à ce statut. 
Dans le domaine de l'aménagement du territoire bolivien, la nouvelle constitution donne leur autonomie aux espaces infranationaux. Cette reconfiguration territoriale favorise la création de régions ayant de nouvelles compétences pour conduire des opérations de planification. La nouvelle gouvernance territoriale se consacre aussi à l'autonomie des départements, des municipalités et des TCO devenus des Entités territoriales indigènes originaires paysannes (ETIOC). Ces ETIOC matérialisent la territorialité des nations préhispaniques qui déborde de la compréhension classique de l'Étatnation. Ces trois types d'autonomies sont des espaces politiques situés au même niveau hiérarchique.

Dès 1998, on décentralise l'administration des chemins bolivienne, qui reste de fait très hiérarchisée. Elle distribue les rôles dans la construction et le maintien des réseaux viaires, à différents niveaux: national (réseau viaire fondamental: RVF), départemental (réseau complémentaire) et municipal (réseau vicinal). Cette gestion s'est maintenue pendant la période plurinationale. Les ETIOC et les municipalités sont formellement chargées du développement de routes vicinales, tandis que les autres types de réseaux sont à la charge du gouvernement central et départemental. Par conséquent, les discussions autour du développement du RVF ne semblent concerner que l'État central.

Les deux projets routiers ayant pour objectif de connecter la principale ville de Cochabamba aux villes des départements de La

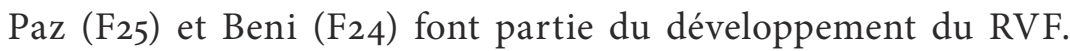
Cependant, on propose que les projets traversent des parcs nationaux et des ETIOC. Les conflits sociaux qu'ils déclenchent permettent de saisir les tensions qui existent entre le discours global environnemental et celui de la fortification de l'État central modernisateur. Néanmoins, ces deux projets procèdent d'enjeux sociaux différents. En effet, le cas du F24 est plus complexe et médiatisé du fait de l'ampleur de la répression des mouvements indigène et environnementaliste opposés au projet routier.

Ces deux extensions du réseau viaire fondamental visent à combler le manque d'articulations entre la ville de Cochabamba et le nord 


\section{Réseau viaire fondamental (RVF) dans le département de Cochabamba}

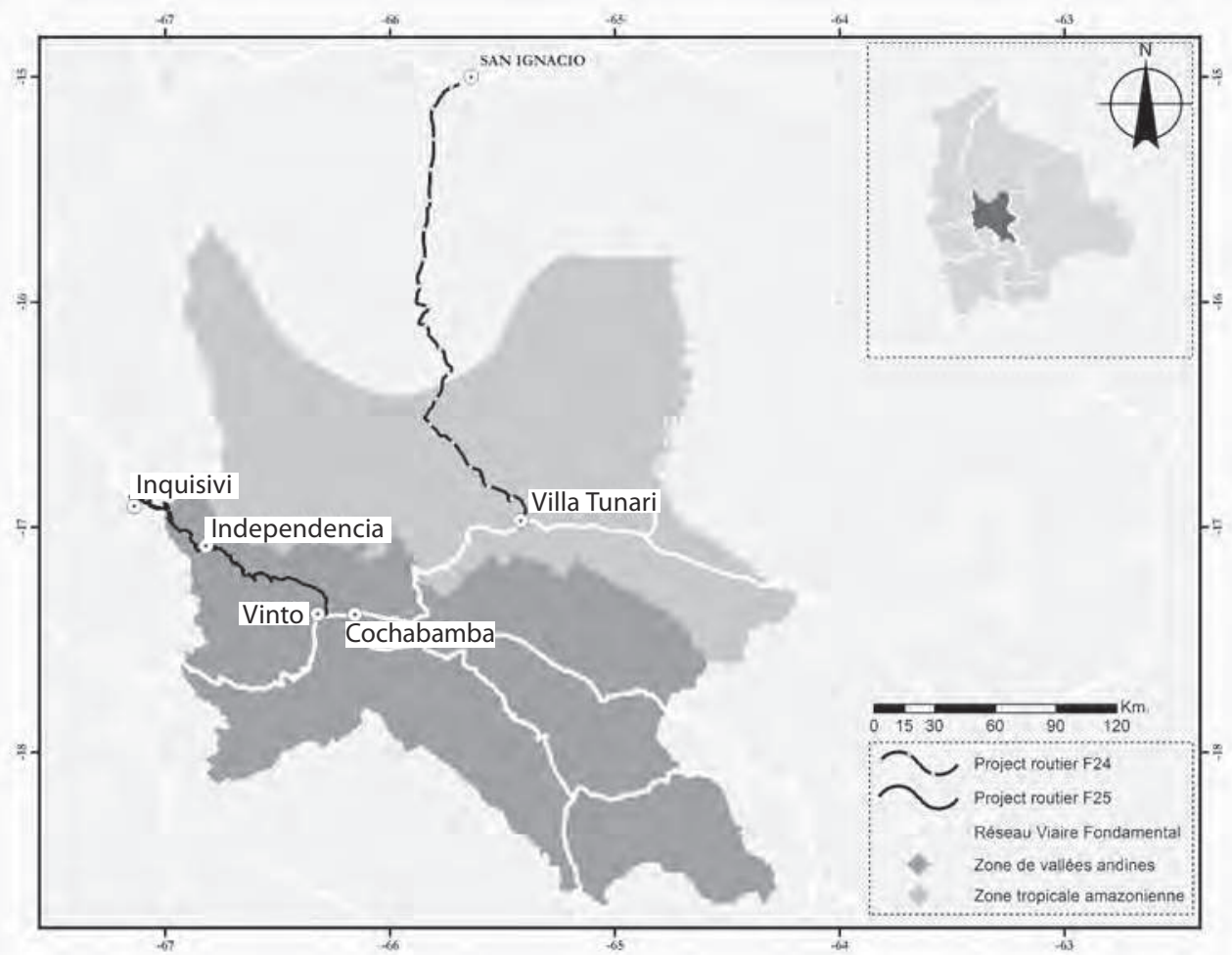

Réalisation: Bianca De Marchi Moyanno et Huascar Ignacio Morales Quintela.

du département. Bien que leurs tracés traversent des ETIOC et des parcs nationaux, ces routes vont traverser différents paysages, l'une partant vers les montagnes et l'autre plutôt vers le sud et la plaine amazonienne. Ces régions sont aussi différentes dans leurs représentations sociales contemporaines, la région tropicale du département étant nettement plus valorisée.

La F25 a comme destination la ville de La Paz. Elle est tributaire d'un relief fortement accidenté. Ce projet routier concerne principalement l'amélioration de chemins déjà existants, le territoire étant traversé par des chemins mal entretenus qui grimpent sur la cordillère 
orientale des Andes. Par contre, le projet de la F24 s'impose de façon hiérarchisée et centrale par rapport au territoire traversé. Il est lié à la logique d'organisation syndicale cocalera ${ }^{3}$, en conflit avec l'espace de protection appelé Territoire indigène et parc national IsiboroSécure (TIPNIS).

Mais le développement des conflits autour des deux projets montre que la logique de subordination au gouvernement central des entités territoriales censément décentralisées persiste. L'idée de l'autonomisation (c'est-à-dire des institutions déhiérarchisées) qui est à la base de l'État plurinational rencontre des difficultés évidentes de mise en œuvre. Lorsque des entités territoriales n'ont pas les mêmes objectifs, les conflits s'intensifient en mettant à rude épreuve le pouvoir central. Dans le contexte de gestion et de concertation autour de la construction des chemins contemporains de Cochabamba, les organismes multilatéraux d'appui au développement, ainsi que les organisations indigènes, environnementalistes et non gouvernementales ont autant d'importance que les autorités étatiques et les entreprises commerciales et productives transnationales.

Les deux projets routiers permettent d'affirmer que la structuration du réseau viaire bolivien est le résultat de rapports de pouvoir entre différents acteurs sociaux politisés, qui y font face et qui s'imposent dans le territoire. Ainsi, les infrastructures s'érigent au rythme des enjeux associés aux acteurs globaux, nationaux et locaux. Ces jeux autour des grandes infrastructures relèguent finalement au second plan les discussions techniques concernant les infrastructures de transport. Les autres voies de transport, par exemple ferroviaires ou fluviales, vont presque disparaître des débats, le transport automobile occupant une place quasi exclusive. Dans l'Amazonie, le transport fluvial continue à être sous-estimé, tandis que l'importance du train se réduit progressivement dans les vallées andines. Depuis la seconde moitié du $\mathrm{Xx}^{\mathrm{e}}$ siècle, les automobiles sont le mode de transport dominant, favorisé par des projets de développement territorial sur le

3. Il s'agit de la population paysanne pratiquant la culture de la feuille de coca. 
continent américain et en Bolivie. Une telle préférence aujourd'hui s'explique donc historiquement et représente une accumulation d'orientations et de décisions d'aménagement, qu'il faut réinterpréter dans le contexte des dynamiques territoriales contemporaines.

Depuis les années 1950, l'État encourage le processus de colonisation de l'Amazonie dans le département de Cochabamba. L'extension des infrastructures routières dans ce type d'espaces est guidée par des intérêts d'exploitation des ressources naturelles. Cependant, à la fin $\mathrm{du} \mathrm{xx}^{\mathrm{e}}$ siècle, la protection de l'environnement émerge du rapport de force entre acteurs sociaux, principalement dans l'Amazonie: l'idée de services fournis par les écosystèmes globalement valorisés s'impose, tandis que les écosystèmes andins sont constamment marginalisés.

On représente ainsi partiellement l'échelle globale par le commerce international des ressources, mais aussi par les parcs nationaux, qui sont liés aux projets internationaux environnementalistes ${ }^{4}$. Les échelles se complexifient avec les espaces de gouvernement infranationaux, comme les départements, les municipalités et les ETIOC qui sont traversés par les projets routiers. Toutefois, le réseau viaire fondamental est un projet de l'État central et son développement est dépendant de la capacité du gouvernement à réussir des alliances et des subordinations politiques avec les gouvernements des aires infranationales. Souvent, les tensions électoralistes et les conflits d'acteurs prennent le pas.

Ces différents jeux sont ancrés dans les niveaux d'organisation politico-administratifs de l'État, tout en étant conditionnés par la pression des environnementalistes et des organisations commerciales. Cette analyse révèle les tensions entre les échelles de la dynamique plurinationale, sous forme de recomposition des différentes aires délimitées et des rapports entre les discours environnementaux globaux et développementalistes d'exploitation des ressources.

4. Sur les projets internationaux de mobilité et de commerce, l'exemple le plus évident est l'Initiative pour l'intégration de l'infrastructure régionale sud-américaine (IIRSA); pour les projets environnementalistes, nous pouvons citer le Corridor AmboróMadidi (CAM). 
On observe donc une sorte de reproduction de la logique frontalière et de la souveraineté républicaine dans les espaces infraétatiques, qui ne se laissent cependant pas rattacher aux logiques transnationales dominantes. Les acteurs qui participent aux conflits du développement routier représentent ces intérêts. Cette approche politique permet de mettre en exergue les tensions sociales et les limites de l'actuelle conception de la mobilité bolivienne.

\section{L'organisation des chemins}

Soulignons que l'analyse présentée est le fruit d'une démarche de recherche spécifique qui cherche à comprendre les conflits dans le développement des chemins boliviens. Ce filtre de lecture ne réussit pas à approfondir les usages quotidiens de la circulation sur les routes ni à développer un regard de proximité sur la production des chemins. On accorde la priorité d'analyse aux acteurs collectifs et à leur capacité de mobilisation politique et discursive. Malgré cela, dans les différents éléments d'analyse exposés, nous pouvons rentrer quelque peu dans l'expérience spatiale du vécu et du quotidien ou, en d'autres mots, de la proximité. Mais pour bien la saisir, il est nécessaire de distinguer différentes échelles d'analyse sociospatiale. Adopter une approche ethnographique et microsociologique, complémentaire à l'approche politique et territoriale mobilisée plus haut, nous permet d'émettre des hypothèses sur le rapport entre les modes de transport et les types de mobilité utilisés.

Pour ce faire, mes réflexions s'appuient sur la notion d'«échelles de voisinage» que propose Lévy (1994). Ces dernières permettent en effet de mieux comprendre comment différentes manières de parcourir les espaces, et notamment les chemins, s'articulent et se confrontent. Les échelles ne renvoient pas seulement à la distance physique, mais aussi à la fréquentation des lieux et des parcours. Cette proposition illustre bien que la mobilité quotidienne (résidence-travail par exemple) constitue une première échelle de mobilité. Cette compréhension, à partir des usages de l'espace, révèle une autre façon de 
produire de l'espace. Ainsi, l'échelle n'est pas qu'une mesure de taille, mais aussi de temps, d'argent et de bien d'autres facteurs. Chaque sujet, à partir de sa façon particulière de mesurer ses espaces de référence, saisit ainsi la valeur et la substance des échelles.

Une autre méthode d'analyse de l'échelle consiste à la resituer au sein d'études paysagères. Si le paysage est conçu à partir de la perception du sujet de son environnement, en tant qu'«interprétation sociale de la nature» (Bertrand, 1995), il devient une occasion de valorisation de l'expérience humaine dans l'espace. Les chemins, malgré leurs niveaux d'attachement formel aux échelles administratives, s'apparentent à une architecture (Berque, 2008) qui permet la lecture des paysages depuis la circulation, où les différentes références spatiotemporelles des usagers se rencontrent. La prise en compte des perspectives et des projections des marcheurs, ainsi que de leurs descriptions techniques et symboliques de l'environnement, peut servir de base de dialogue entre acteurs dans la mise en œuvre de nouveaux itinéraires et formes de mobilité. Elle permet aussi de mieux cerner les diverses expectatives en matière d'innovation et d'invariants de la mobilité sur les territoires traversés.

Ces approches sont importantes, car elles peuvent être reprises comme base d'action pour un aménagement et un développement territorial inclusifs et intégraux. L'espace quotidien peut participer aux projets en tant que dimension vivante du territoire. Ainsi, le développement routier peut s'appuyer sur les échelles de proximité constituant les niveaux de référence pour favoriser la participation citoyenne et politique.

Celle-ci est aussi tributaire de modes d'expression politique proprement boliviens, où la participation des acteurs locaux et des citoyens à la sphère publique est encouragée par différents mécanismes et procédures. Au sein de la nouvelle constitution de 2009, la consultation "préalable, libre et informée» est encouragée, surtout lorsque les interventions affectent des aires dédiées aux peuples indigènes (ETIOC). Cependant, l'application de cette procédure a tendance à instrumentaliser la population indigène et à légitimer les 
projets officiels, voire à accroître la monétarisation des échanges fonciers.

De fait, les consultations n'incorporent pas formellement les acteurs des différentes échelles. Malgré cela, une lecture critique des résultats des consultations peut donner quelques pistes de compréhension de leurs interactions et de leurs conflits. Bien qu'il existe cet engagement pour la participation, les acteurs politiques s'imposent et l'échelle du quotidien reste difficile à saisir.

Le défi est non seulement de remettre en question le caractère sporadique et légitimant de la petite échelle, mais aussi de concevoir les routes en tant qu'espaces de communication entre les différents réseaux et niveaux de mobilité qui composent un territoire, où les limites sont plutôt zonales et poreuses. En Bolivie, ce regard renforce les principes clés de l'État plurinational, car les acteurs des différents niveaux d'autonomie doivent nécessairement dialoguer autour de rapports déhiérarchisés et processuels. Mener des projets routiers demande donc d'impliquer différents acteurs, de l'amont (phase du dessein et du dessin) à l'aval (gestion des flux). Ces acteurs pourraient être impliqués au moment de la conception des routes par exemple, mais ils peuvent aussi devenir des participants transversaux et processuels qui se maintiendront comme parties dans la gestion des flux.

Il est aussi souhaitable d'établir les lieux traversés par le tracé comme ancrages particuliers, pour faciliter le dialogue de la diversité d'acteurs qui participent de la construction du territoire. La géographie autour du projet pourrait être la base de la construction de scénarios et d'hypothèses prospectives dans la prise de décision collective à partir de l'échelle locale, mais aussi en dialogue avec les autres échelles. De cette façon, le paysage peut être mobilisé en tant que médiateur (Loza, 2008) en vue de valoriser la diversité de rencontres entre les modes de mobilité existants (De Rijck et Peemans, 2004). Finalement, un projet routier pourrait aussi devenir un moteur du développement territorial plurinational. 



\author{
TROISIÈME PARTIE \\ LES ENJEUX RÉFLEXIFS \\ ET MÉTHODOLOGIQUES DU TERRAIN
}





\title{
CHAPITRE 8
}

\section{Les outils d'urbanisme, entre modernité et développement}

\author{
Claire Simonneau
}

Comment, du point de vue du chercheur, se décline la question d'une recherche sur les outils d'urbanisme sur les terrains africains ${ }^{1}$ ? Cette question classique de la démarche scientifique s'impose tout particulièrement aux chercheurs occidentaux étudiant les outils d'urbanisme, pour lesquels la distance vis-à-vis de leur objet de recherche ne va pas de soi. Il serait plus juste de parler d'un singulier mélange de distance et de proximité, d'une double dynamique à la fois endogène et exogène. Cette question traverse dans une certaine mesure les études urbaines africanistes et les recherches en urbanisme portant sur les pays du Sud. Elle peut être ainsi traitée sur deux plans. Elle pose un problème méthodologique, que traitent notamment la socioanthropologie et l'ethnologie $e^{2}$. Au-delà il s'agit surtout d'un problème épistémologique, de cadre de pensée, comme la récente critique postcoloniale l'a démontré.

1. Le titre du chapitre fait référence à l'ouvrage de Jennifer Robinson: Ordinary cities: between modernity and development, Routledge, 2006.

2. Cet aspect est plus amplement analysé par Léandre Guigma au chapitre $10 \mathrm{du}$ présent ouvrage. 


\section{Les outils d'urbanisme : importés ou adaptés?}

La question de l'altérité, posée par la relation que le chercheur entretient avec ses terrains et ses objets de recherche, se décline d'une manière particulière en urbanisme et dans les contextes africains. Le cas des outils d'urbanisme ${ }^{3}$ permet d'explorer cette singularité. En effet, ces outils sont souvent hérités de la période coloniale. Ils accompagnent un important dispositif légal et technique d'encadrement de la ville, en particulier de sa production foncière, repris quasi intact par les États africains à leur indépendance. L'aide au développement postindépendance a également contribué à mettre en place des outils conçus en Occident dans le cadre de transferts de technologies, notamment institutionnelles. En ce sens, la «greffe» est une modalité très répandue de mise en place d'outils d'urbanisme au sein des administrations des villes africaines. Cependant, ces greffes ne prennent pas place dans des contextes vierges et anhistoriques; au contraire, ces administrations ont une longue histoire, faite d'empilements de modèles qui se cumulent, voire qui produisent des contradictions (Darbon et Crouzel, 2009).

Prenons l'exemple d'un cadastre simplifié au Bénin, le Registre foncier urbain $(\mathrm{RFU})^{4}$. On a conçu cet outil, comme beaucoup d'autres, dans le cadre d'un projet de développement financé par la coopération française, avec l'aide d'un assistant technique étranger. Ce projet de développement particulier s'inscrit dans une réforme de plus large portée menée par les institutions internationales qui font la promotion de la notion de gestion urbaine dès le milieu des années

3. Daniel Pinson définit trois «noyaux durs» des objets de recherche en urbanisme: le territoire, le projet (y compris ses techniques et ses instruments), ses représentations (2004).

4. Le Registre foncier urbain (RFU) est un cadastre municipal informatisé: il est composé d'un plan parcellaire et d'une base de données fiscales, foncières et urbaines. Il peut jouer trois rôles: amélioration de la fiscalité locale, meilleure connaissance du patrimoine foncier de la commune et de l'occupation du sol, et outil d'aide à la décision, en liant la base de données à un système d'information géographique. Ce chapitre fait référence à une recherche doctorale de l'auteure soutenue en 2015 à l'Université de Montréal. 
1980. La Banque mondiale a d'abord théorisé cette nouvelle stratégie, qui s'inscrit dans l'idée que le développement passera par le déploiement de l'économie de marché, le retrait de l'État et l'action concertée entre acteurs urbains, incluant les autorités publiques centrales et décentralisées, le secteur privé et la société civile. Les instruments traditionnels de la planification sont alors délaissés au profit des outils de gestion (des finances publiques, des investissements, de l'information foncière, etc.) inspirés de ceux du secteur privé et des théories du New Public Management. La conception du cadastre simplifié béninois est donc encastrée dans une théorie plus large de la gestion urbaine pensée depuis l'Occident et y faisant référence. Aussi, l'objet de recherche n'est pas complètement exotique pour le chercheur occidental et peut même être relativement familier à celui-ci. Cependant, à une échelle plus fine, on a déterminé les procédures de fonctionnement de l'outil selon un certain nombre de paramètres proprement béninois, comme le système de la fiscalité locale, les institutions existantes, ou encore la disponibilité des données cartographiques.

Les dynamiques endogènes et exogènes sont également entrelacées dans le cheminement historique de l'instrument. C'est bien dans le cadre d'un projet de développement exogène que le RFU a été conceptualisé à la fin des années 1980, mais cette conception s'est effectuée à l'intérieur d'un service ministériel national, avec un assistant technique français entouré d'une équipe de cadres béninois. De plus, alors que c'est un financement international qui a permis l'installation de l'outil dans toutes les villes concernées, ce financement est partout terminé, de sorte que la mise en œuvre est aujourd'hui assurée par les administrations locales, selon les routines propres à elles, avec la collaboration d'experts, d'institutions publiques et d'entreprises privées, tous béninois.

Dans une telle recherche, comment appréhender ce singulier mélange de distance et de proximité, d'endogène et d'exogène? Cette question doit être pensée sur deux plans: l'un, méthodologique et l’autre, épistémologique. 


\section{Des questions de méthodologie et d'épistémologie}

La place du chercheur et la distance culturelle sont des points de discussion méthodologique fondamentaux pour les sciences sociales. L'anthropologie et l'ethnologie ont largement abordé les problèmes de méthode pour comprendre le point de vue et l'organisation de sociétés étrangères. La sociologie qualitative a plutôt traité l'étude des contextes proches, mais en soulignant l'altérité provoquée par les effets d'image renvoyée par le chercheur, liés aux classes sociales, à l'âge, aux milieux socioprofessionnels, etc. Cette littérature fournit ainsi plusieurs balises méthodologiques pour s'assurer d'une rigueur scientifique dans l'étude qualitative de terrains étrangers ou exotiques.

La première de ces balises réside dans l'imprégnation du contexte: il s'agit, souvent par une insertion prolongée dans le milieu d'étude, de s'imprégner des codes de bienséance, de se familiariser avec les préoccupations de la vie quotidienne et, finalement, d'approcher le système de sens du milieu étudié. De plus, le chercheur doit prendre conscience de sa propre culture d'origine et de ses préjugés, ainsi que de l'image qu'il renvoie dans le milieu étudié. Il s'agit alors de déterminer les éventuelles perturbations engendrées par la présence et le travail du chercheur, pour pouvoir les limiter, les intégrer à l'interprétation, ou en tirer parti. Enfin, il est nécessaire de faire preuve d'honnêteté méthodologique, en précisant les conditions concrètes de l'enquête (le temps passé sur le terrain, les informateurs, les présupposés théoriques à partir desquels l'interprétation est faite). Ainsi, aucune situation d'enquête n'est totalement neutre, aucune collecte de données n'est absolument objective. Seules l'analyse et la restitution des conditions de production de la connaissance autorisent à prétendre à la validité des résultats.

Un constat fréquent fait sur les terrains urbains africains par les chercheurs étrangers (souvent occidentaux) relève du statut que leur donnent les enquêtés: l'amalgame entre chercheur et consultant d'agence de développement est en effet fréquent sur ces terrains 
rompus à l'aide au développement. Cette assimilation peut ouvrir des portes sur le terrain, mais aussi en fermer.

Il est également possible de mobiliser d'autres outils de collecte de données permettant de contourner les obstacles engendrés par le statut du chercheur. Reprenons le cas d'une recherche sur la mise en œuvre du RFU au Bénin: la collecte des données par les entretiens a été complétée par l'observation des organisations (matériel à disposition, organisation des bureaux, relations entre fonctionnaires et usagers), des relevés de terrain (morphologie urbaine, architecture, matériaux, etc.) et la production de recensions systématiques (comptage, repérage, cartographie sommaire, prix fonciers affichés ou annoncés). Enfin, l'utilisation de données et d'analyses de seconde main, comme des mémoires de recherche issus des universités locales, peut également permettre de vérifier la validité des autres sources ou de les compléter.

Cependant, dans le contexte particulier d'une recherche sur des outils d'urbanisme, le problème peut dépasser la dimension méthodologique. Il revêt également une dimension épistémologique, liée à la formulation du problème de recherche par le chercheur et par les informateurs sur le terrain.

La critique postcoloniale a contribué à révéler cette dimension épistémologique et en a montré les implications pour la recherche urbaine sur le continent africain. Le postcolonialisme est un mouvement de pensée qui s'attache à comprendre et à dépasser les structures de domination forgées par le phénomène colonial, notamment autour des questions de construction identitaire et de production de savoirs ${ }^{5}$. Depuis le début des années 2000, un nombre croissant de chercheurs en études urbaines se revendiquent de ce mouvement de pensée et

5. Le postcolonialisme désigne plus largement un courant intellectuel critique apparu dès le milieu du $\mathrm{xx}^{\mathrm{e}}$ siècle. Il propose une démarche visant à comprendre les conditions de la production des savoirs sur soi et sur l'autre dans un contexte de domination, ainsi que la marge de manœuvre - en matière de pensée et d'action - dont disposent les populations subalternes. Cette démarche a été appliquée dans de nombreuses disciplines et à de nombreux sujets, et en particulier aux enjeux d'identité et de production de connaissances. 
mobilisent certaines de ses méthodes et approches conceptuelles critiques dans leurs recherches à l'instar Mbembe et Nutall (2004), Murray et Myers (2006), Myers (2011), Robinson (2006). Simone (2004) et Watson (2009). Ces auteurs ont mis en évidence la pensée colonisatrice qui imprègne encore les villes africaines et les représentations occidentalo-centrées qui en découlent, au point de formater les politiques urbaines.

\section{Les critiques de la recherche urbaine africaniste}

Le premier apport des auteurs se revendiquant du courant postcolonial a été de montrer que la recherche urbaine africaniste est formatée par un double paradigme, de la modernité et du développement, ce qui constitue un frein à la compréhension des dynamiques urbaines africaines, pour paraphraser Robinson (2006).

Le paradigme de la modernité se caractérise par la croyance dans le progrès et par la confiance dans le fait que la science et la technologie permettront une amélioration des conditions de vie, au fil du temps et pour les générations futures. Le paradigme du développement s'inspire également de l'idée de progrès, mais s'applique plus directement aux pays dits «du Sud». Il prétend définir un état souhaitable pour un pays, celui du développement, défini principalement sur le plan économique dans les années 1960-1970, auquel se sont ajoutés des critères de développement humain (éducation, santé, niveau de vie) dans les décennies 1980-1990, puis de durabilité dans les années 2000. Il renferme ainsi l'idée d'un changement délibéré et dirigé. Ce double paradigme, entretenant des liens étroits avec la pensée coloniale, formate de manière puissante les productions de connaissances sur les villes non occidentales en général, et sur les villes d'Afrique subsaharienne en particulier.

Le double paradigme modernité/développement implique deux grands biais dans les approches du fait urbain en Afrique subsaharienne. Premier biais, une partie importante de la littérature scientifique et des approches techniques décrit ces villes dans les termes du 
manque: on cherche à identifier ce qui manque à ces villes pour qu'elles fonctionnent et se développent (des liens économiques globaux, une bonne gouvernance, des infrastructures adéquates, de l'emploi dans le secteur formel, etc.). La pensée urbaine dominante utilise ainsi comme référence, de manière plus ou moins explicite, le modèle des villes occidentales. En conséquence, de nombreux chercheurs et praticiens peinent à considérer les villes africaines et certaines de leurs caractéristiques (quartiers informels, extension spontanée de la ville, statuts fonciers multiples, etc.) comme normales, ordinaires et non problématiques. On évalue généralement ces villes en référence à l'urbanisation européenne, et en ce sens, elles sont régulièrement décrites comme des anomalies ou des aberrations. Finalement, le discours sur ces villes oscille entre deux pôles, l'un optimiste et l'autre pessimiste: dans le premier cas, les villes africaines sont présentées comme des situations de rattrapage des degrés et des formes d'urbanisation en Occident; dans le second cas, comme des situations apocalyptiques.

Deuxième biais, et dans la continuité du précédent, la production de savoirs sur les villes africaines est étroitement liée à l'élaboration de politiques publiques dans une perspective de solutions opérationnelles. Pieterse (2009) parle ainsi d'un "policy-fix genre», d'ailleurs en grande partie financé par les agences d'aide au développement. Ce type de recherche contribue au renouvellement récurrent des ingrédients du développement urbain promus par les agences d'aide: ces dernières décennies, ces ingrédients seraient la libéralisation économique, l'économie de marché et la démocratisation/décentralisation. Or, ces solutions se révèlent souvent inadaptées, puisqu'on les élabore dans un cadre de référence occidentalo-centré, avec des connaissances très limitées sur les dynamiques réelles de l'urbanisation et du jeu d'acteurs urbains. Par exemple, les stratégies de développement ont présupposé à tort la cohérence des communautés urbaines, et par conséquent la capacité d'une société civile à prendre en charge l'aménagement.

Les politiques publiques urbaines apparaissent particulièrement formatées par ce double paradigme. L'aide au développement et sa 
boîte à outils, tout comme l'influence de ces paradigmes sur les modes de pensée des décideurs africains, conduisent à des décisions de politiques publiques devant répondre aux manques, déterminés à partir de constats souvent formulés par des théories et des méthodes allochtones pouvant être très déconnectées des contextes locaux. L'émergence d'outils et d'approches réflexives innovants et adaptés s'en trouve alors freinée.

Dépasser ce paradigme est un défi important tant il est intégré par un certain nombre de cadres et d'agents administratifs, d'experts africains, voire parfois par les "populations bénéficiaires» ellesmêmes, selon le vocabulaire consacré. Une partie importante de ces acteurs connaissent et manipulent le vocabulaire du développement, adoptent une organisation du discours apte à formuler un projet de développement et prennent comme référence (explicite souvent) le modèle occidental. De nombreux auteurs y voient un effet pervers et parfois inattendu de l'aide au développement.

Cependant, une telle critique ne doit pas refuser aux sociétés africaines (lesquelles incluent les administrations publiques) des aspirations sociales, des idéologies politiques ou des référentiels de politiques publiques. Les valeurs, les aspirations et les idéaux que véhicule tout modèle de ville et de société trouvent un écho en Afrique comme partout ailleurs dans le monde. Dénigrer ce fait serait tomber dans un double piège: celui du manichéisme d'un côté, qui se limite à la dénonciation et à la traque des restes de colonialisme dans les sociétés et les politiques africaines; celui du culturalisme idéologique de l'autre, en quête d'une authenticité africaine essentialisée, aux expressions socioculturelles précoloniales, ancestrales et autochtones. Ainsi, les aspirations sociales à une forme occidentale de modernité sont à prendre au sérieux, au même titre que les fondements ancestraux des règles d'accès à la terre dans certains quartiers urbains.

Le problème pointé par la critique postcoloniale réside plutôt dans le rapport que la recherche entretient avec les modèles. La référence à un modèle idéal est en effet problématique dès lors qu'elle formate la recherche, qu'elle oriente les questions posées et qu'elle biaise les 
conclusions de recherche. Ces auteurs du courant postcolonial plaident donc pour une recherche engagée, qui contribue à mettre au jour les réalités urbaines, sociales, économiques et politiques des mondes urbains, et ce, dans toute leur complexité.

\section{Une stratégie de recherche renversée}

Le courant postcolonial de la recherche urbaine encourage finalement un retournement des stratégies de recherche qui peut se résumer comme suit: plutôt que de décrire les villes africaines dans les termes du manque, tentons de comprendre ce qui fonctionne dans ces villes, d'identifier celles et ceux qui produisent la ville, matériellement et symboliquement, de mettre en lumière les logiques qui concourent à leur construction et leur fonctionnement quotidien. Ce retournement apparaît comme fondamental sur le plan épistémologique, mais aussi sur le plan pragmatique, dans une perspective d'élaboration d'un urbanisme ancré au plus près des réalités urbaines locales. Plusieurs éléments permettent d'opérer ce retournement de pensée:

- Considérer ces villes comme ordinaires, et non comme la version ratée d'un modèle. Ce positionnement implique de faire basculer le raisonnement, en portant attention non plus seulement aux blocages et aux éléments manquants, mais aussi et surtout aux éléments structurants et aux logiques discrètes de régulation qui produisent, d'une certaine manière, la ville.

- Contextualiser ces recherches, autrement dit considérer ces situations urbaines pour ce qu'elles signifient dans leur contexte, et non en référence à ce qu'elles devraient être selon un modèle préétabli. Ceci signifie prendre en compte la complexité et la profondeur historiques et géopolitiques (position géographique, rapport à la colonisation, à la mondialisation) de ces situations.

- Considérer les habitants comme des agents actifs de la construction physique et symbolique de la ville plutôt que comme de simples victimes d'un processus non contrôlé. Dans le même mouvement, 
il est nécessaire de prendre au sérieux l'informalité et les pratiques urbaines métissées et de poser l'hypothèse que l'ensemble de ces pratiques peut constituer une forme innovante d'urbanisme ${ }^{6}$.

- Adopter une approche relationnelle ou interactionniste entre les acteurs urbains, au-delà des catégories de types formel/informel, public/privé, moderne/coutumier.

Finalement, les approches postcoloniales posent l'hypothèse d'une complexité et d'une hybridité des villes africaines et de leur régulation. Dans le sillage de l'anthropologie du développement et des sciences politiques, elles proposent comme méthode d'exploration de suivre les processus locaux d'appropriation des modèles extérieurs, impliquant d'étudier leurs logiques de mise en œuvre et souvent leurs déformations. Sur ce point particulièrement, la mise en œuvre d'outils d'urbanisme représente un objet de recherche fécond pour mettre en lumière ces hybridations.

Dans cette perspective, il semble également intéressant d'adopter une approche multidisciplinaire, en mobilisant conjointement des travaux issus des études du développement et des recherches urbaines africanistes portant par exemple sur les modes de vie urbains. Ces dernières peuvent en effet donner des clés d'interprétation des mécanismes de métissage entre les différents modèles dont la société urbaine est le creuset; elles permettent aussi de mieux considérer les répercussions de la globalisation sur les pratiques urbanistiques, sur les aspirations et les représentations socioculturelles, ou encore sur les modes de vie urbains, généralement peu traités par les études de développement. En s'intéressant de près aux citadins, de telles approches peuvent finalement donner des pistes de réflexion et d'action pour les chercheurs comme pour les professionnels de l'urbain.

6. Cette perspective est particulièrement développée dans les recherches de Gavin Shatkin portant sur les pratiques urbanistiques ordinaires et quotidiennes, ce que l'auteur nomme les «actually existing urbanisms» (2011). 


\section{CHAPITRE 9}

\section{Un nécessaire bricolage méthodologique}

Divya Leducq

S’appuyant sur des expériences de recherche empiriques menées dans différentes villes du sud de l'Inde depuis 2007, et à Hanoi au Vietnam depuis 2010, ces réflexions ont pour but de comprendre quelles sont les dynamiques urbaines faisant suite à la mise en œuvre des lignes d'action visant soit à accroître l'attractivité et le développement économique, soit à impulser une réorganisation spatiale d'une grande région métropolitaine. Ces deux objectifs se rejoignent parfois et laissent apparaître une hypothèse de travail pour le jeune chercheur en aménagement et urbanisme.

Ainsi, de nombreux auteurs et spécialistes des sciences humaines et sociales soutiennent qu'un bricolage méthodologique est incontournable pour recueillir des données de terrain et contribuer à documenter son objet de recherche. Cette hypothèse s'apparente à un positionnement du chercheur face à un terrain qui est éloigné géographiquement de son quotidien, mais dont la distance peut aussi se mesurer par la différence linguistique, culturelle ou religieuse. Les cadres de référence, les techniques ou encore les acteurs de l'urbanisme recouvrent des réalités parfois aux antipodes des connaissances préalables du chercheur et il convient souvent de les examiner, afin 
d'éviter des maladresses de lecture ou les incompréhensions d'un terrain que l'on chercherait avant tout à s'approprier.

\section{Une littérature éclairante}

Remettre en question l'émergence des systèmes territoriaux de l'innovation informatique à Pune ou analyser la durabilité du dernier plan directeur de Hanoi a nécessité préalablement, et en guise de défrichage du terrain à venir, d'examiner des objets d'étude, comme le cluster innovant ou les documents de planification stratégique. Il a fallu aussi considérer les caractéristiques des espaces d'étude (dimensions urbaine, architecturale, économique, sociologique, démographique, immatérielle...), et a minima les données qui étaient disponibles dans la littérature blanche (scientifique) ou la littérature grise (presse, rapports). Ces deux contextes urbains, bien qu'incomparables en euxmêmes, présentent au moins deux caractéristiques communes. Ce sont des espaces en plein essor et qui connaissent des mutations rapides. Documenter des transformations urbanistiques en cours demande un travail empirique sur place, lequel s'avère incontournable si l'on souhaite les saisir au plus près de leur actualité.

Presque aussitôt et avant mon arrivée en ville, j'ai fait face à plusieurs limites ou contraintes qui m'ont incitée à me mettre dans la peau d'un chercheur-bricoleur, à créer avec les moyens du bord et à me détacher partiellement du chercheur qui élabore plus strictement son projet en amont (Lévi-Strauss, 1962). Il fallait saisir la complexité d'espaces urbains étrangers où les tendances lourdes de l'organisation spatiale de villes éloignées impliquent de l'inspiration et de l'ingéniosité afin de créer et tester des outils qui puissent faire parler les idées et les objets. Les chercheurs confirmés soulignent que le terme «bricolage» - qu'il soit méthodologique ou plus expérimental - aura le défaut de déplaire par le caractère amateur de la démarche scientifique, mais qu'il est en même temps porteur d'originalité et de nouveauté dans la production scientifique. Il permettrait de faire évoluer des démarches et des questionnements par la confrontation et le test 
ou l'adoption de nouvelles solutions, plus ou moins pertinentes, se référant à un «système $\mathrm{D}$ » (débrouillardise) que l'on met souvent en œuvre afin de "faire parler» son terrain et d'obtenir une donnée exploitable (Saint-Martin, 2002). Gumuchian et Marois (2000) montrent ainsi l'importance de laisser la place à une double démarche, à la fois théorique et appliquée.

En tant que jeune chercheure, j'ai tenté de construire une boîte à outils adaptée à chaque question de recherche, et ce, afin de faire face aux problèmes d'accès et de construction de données, en m'inspirant des outils d'investigation existants, dont la pertinence d'utilisation varie en fonction du contexte et des catégories de personnes enquêtées, urbanisants ou urbanisés. Ainsi, l'observation récurrente et l'éthologie des lieux et des espaces, les méthodes d'entretien semidirectif et de questionnaire, les outils d'analyse des espaces publics apparaissent incontournables. Par ailleurs, les "ficelles du métier» qu'évoque Becker (2002) donnent des pistes pour sortir de la monographie de territoire tout en essayant de généraliser des conclusions à partir de l'étude d'un ou de plusieurs cas particuliers.

\section{L’approche qualitative}

Parallèlement à ce bricolage méthodologique, certains problèmes semblent récurrents dans les travaux de recherche portant sur de très grandes villes, qu'il s'agisse de la surface couverte, de leur démographie, parfois impressionnante, ou encore de l'enchevêtrement de leurs systèmes administratifs, socioéconomiques et politiques. Comment tenter de rendre compte de la complexité urbaine? À quelle échelle évoquer les problématiques urbaines (transport, habitat, énergie...)? Comment concilier le lien entre le global et le local en maintenant du sens, sans se perdre dans l'exhaustivité des situations, tout en comprenant où et avec qui cela se passe? Travailler à l'échelle des quartiers peut s'avérer dangereux, car il n'existe pas de quartier sans ville ou agglomération métropolitaine. Parallèlement, ce travail minutieux est indispensable à la compréhension des références, des valeurs et 
des aspirations des individus dans leurs espaces de vie. La question du bricolage revient alors dans le traitement efficace des données recueillies via un flot d'informations acquises aux différentes échelles complémentaires de la planification urbaine.

Travailler sur des espaces en développement ou des villes en émergence, c'est aussi se demander comment instaurer un dialogue - si possible itératif - avec les acteurs et les partenaires du développement, des plus hautes autorités nationales ou internationales au simple citoyen.

Il paraît important d'évoquer les problèmes d'expression et de sémantique que j'ai rencontrés sur mes terrains indien ou hanoïen. En effet, ceux-ci se posent à l'écrit comme à l'oral, en amont de la construction du problème ou des enquêtes, sur le terrain, et enfin au retour de la journée de travail, lors de la retranscription et de l'analyse des résultats. Ce verrou sémantique revêt une triple dimension: il touche en même temps à la langue, à la culture et au positionnement même du chercheur sur son terrain.

Un autre problème vient de la confrontation entre le choix du positionnement théorique de recherche et le champ disciplinaire mobilisable des études urbaines, des études sur le développement, de l'aménagement du territoire, de l'architecture et de toutes les autres disciplines ayant trait aux recherches urbaines (géographie, économie, sociologie, sciences politiques...), qui contribuent à alimenter les débats sur les formes et la gouvernance urbaines actuelles. Autant de corpus qui diffèrent également dans les littératures anglophone et francophone à propos des villes durables ou de l'économie de la connaissance. Cela complexifie dangereusement les acceptions et les définitions que l'on peut avoir de tels notions ou concepts, et donc du vocabulaire sur lequel s'appuieront les recherches.

Lors d'un travail en terrain étranger, nous composons bien souvent avec une langue qui n'offre pas les capacités et les libertés de notre langue maternelle. D'une part, le problème de l'environnement ou de la ville se pose, de même que celui des mots, car ils expriment une vision socialement et culturellement située. D’autre part, l'(in)traduc- 
PHOTO 9.1

\section{Hanoi, les défis d'une dynamique métropolitaine en construction}

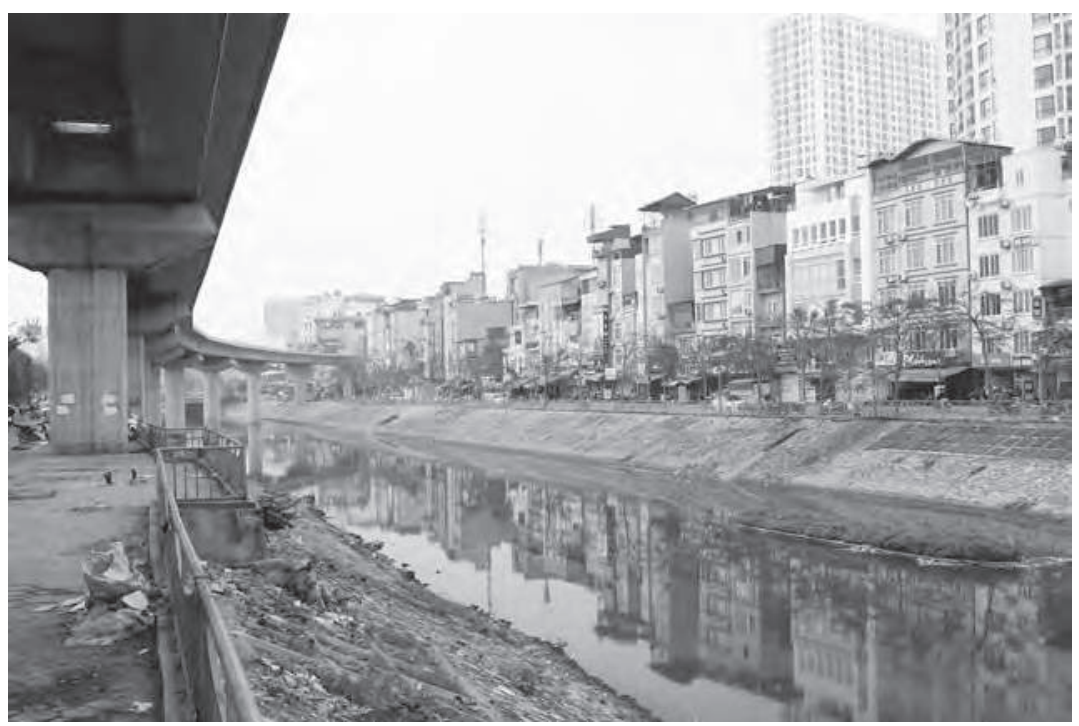

Photo: Divya Leducq, 2016

tibilité des idées, des concepts et des notions d'une langue à une autre implique une perte de sens ou de nuance. L'usage de l'anglais permet de se comprendre dans certaines sphères, mais le passage par les langues vernaculaires et locales comme le vietnamien, le malayâlam, le marâthî ou le tamoul s'avère rapidement incontournable et nécessite la présence d'un traducteur. Par ailleurs, l'enquête scientifique auprès de la population n'est pas aisée en Inde, où elle peut apparenter le chercheur à un journaliste; de même, à Hanoi, de nombreuses barrières, comme les autorisations officielles, ont pour objectif d'éviter la mise en présence directe du chercheur avec la population.

Le chercheur, y compris natif, est dans la très grande majorité des cas étranger sur son terrain. Il travaille dans un milieu auquel il n’appartient pas, mais avec lequel il est amené à se familiariser afin de pouvoir le mettre en débat. Au Vietnam, la particularité provient de l'État communiste et de l'omniprésence des Comités populaires comme partie prenante principale. De ce fait, il est souvent difficile 
de rendre compte du sens et de l'utilité de certaines approches multiacteurs lors des entretiens avec des acteurs de l'aménagement et de l'urbanisme. En effet, l'examen des projets urbains et urbanistiques fait régulièrement face aux justifications des logiques d'une croissance néolibérale et des effets attendus. Les obstacles qu'on rencontre, dans l'élaboration d'un corpus de données, peuvent être liés au chercheur et à ce qu'il accepte ou non de révéler. De même, la recherche est tout autant tributaire de ce que les acteurs sont en mesure de dévoiler quant aux futurs urbains qu'ils construisent, à leur vision de la ville idéelle ou idéale et des projets qu'ils conçoivent - plus ou moins démocratiquement - pour y parvenir. Dans la réalité, les multiples aspects des coalitions d'intérêt ou de la corruption sont difficilement reconnaissables ou quantifiables, et opposent régulièrement un défaut de preuves tangibles pour le scientifique. La programmation des projets urbains implique une divergence de points de vue, des rivalités, des tensions, qui sont la source de tout autant de conflits potentiels ou latents. Lors de la conférence inaugurale des journées de l'APERAU 2014 à Montréal, Annick Germain, sociologue canadienne, insistait sur la nécessité de "comprendre les phénomènes avec empathie avant de faire preuve de discernement critique»; peut-être est-ce là aussi un appel à un nécessaire bricolage épistémologique et méthodologique.

En cherchant à mettre en regard les spécificités de l'urbanisme de villes situées en Inde (Pune) ou au Vietnam (Hanoi), j'ai été amenée à me pencher sur les modalités et les tendances de fond à l'œuvre derrière les processus observables. Ainsi, il apparaît que des questionnements se posent autour du creusement des inégalités sociospatiales lié à la construction des nouvelles centralités économiques et des zones multifonctionnelles dans la ceinture périurbaine. Les temporalités stratégiques des acteurs et leurs décisions en matière d'aménagement ou de politiques publiques d'innovation soulèvent d'autres interrogations, de même que la naissance de pratiques d'action collective dans le cadre de la contestation foncière.

Imprégné de la notion de développement urbain durable, le monde tend à se doter de modèles que le chercheur décrypte en carac- 
térisant les vecteurs de mobilité et les modes de circulation des références et des idées qui inspirent les pratiques locales, par exemple grâce à l'influence des cabinets d'urbanisme transnationaux dans l'avènement des figures du parc technologique et scientifique, de la ville satellite ou encore des écocités vertes. On cherche également à analyser les facteurs qui contribuent à renforcer ou à affaiblir la capacité des institutions nationales, régionales et locales à maîtriser l'aménagement de leur territoire.

Enfin, que ce soit en Inde ou à Hanoi, le statut de la recherche en sciences sociales semble être en cause. En dehors des établissements d'excellence nationale et internationale, l'enseignement supérieur s'appuie très peu sur la recherche. La question méthodologique qui se pose vient du fait que les résultats, parfois critiquables et grossiers, importent souvent davantage que les moyens. Ainsi, il n'est pas rare de constater que les conclusions d'articles ou d'ouvrages de recherche sur le développement des territoires - inscrits dans une dynamique de rattrapage ou d'émergence - reprennent en partie des rapports de cabinets de consultants ou des statistiques officielles non fiables.

Il faut donc essayer de construire sa propre boîte à outils, tout en assurant la cohérence des méthodes empruntées afin d'apporter des données fiables acquises honnêtement. Se pose enfin la question de la pertinence des résultats et de la validation des méthodes, qui amène indirectement à élaborer une méthodologie expérimentale afin de déconstruire les fausses données quantitatives et qualitatives disponibles, pour reconstruire l'information. Le temps passé sur le terrain ne semble jamais suffisant au vu de l'ampleur des enquêtes nécessaires à la reconstruction des processus et de la réalité. Les barrières culturelles ou linguistiques mettent aussi parfois du temps à tomber et biaisent, de ce fait, des résultats dont la lecture est dans certains cas trop hâtive, le chercheur étant dans l'obligation de publier ou de diffuser sans délai ses travaux. 



\title{
CHAPITRE 10
}

\section{Les identités du chercheur}

\author{
Léandre Guigma
}

Les identités du chercheur peuvent être multiples en fonction des spécificités des acteurs du terrain étudié et de l'image reflétée par le chercheur lui-même. Les terrains de recherche concernés sont constitués de quartiers non lotis, situés à la périphérie nord-ouest de Ouagadougou (capitale du Burkina Faso): Bissighin et WatinomaNoghin-Basnéré (WNB). Précisons tout d'abord que l'urbanisation de Ouagadougou se caractérise par la superposition d'une trame lotie sur un espace déjà habité, localement désigné par le terme "non loti». Les citadins qui y résident ont acquis leurs terrains auprès des chefs traditionnels et des propriétaires terriens locaux. Ils y ont construit leurs habitations sans l'autorisation de l'administration. La plupart des habitants de ces quartiers non lotis sont dans l'attente d'une régularisation foncière par le lotissement. De ce fait, les zones d'enquête font l'objet de mutations rapides, tant sur le plan de l'occupation spatiale que sur celui de la planification et de l'interprétation des projets d'aménagement urbain en cours ou à venir.

Les stratégies des acteurs et leurs discours respectifs de légitimation de leurs actions ont été analysés en fonction des politiques institutionnelles et des dynamiques sociales en cours dans les quartiers non lotis, suivant une logique interactionniste. Quelles ont été les difficultés identitaires rencontrées par le chercheur sur ses terrains 
d'enquête, lors de ses entretiens avec les acteurs du terrain, puis au cours de ses séances d'observation à des ateliers sur le même sujet? Quels moyens ont permis d'atténuer, de contourner ou d'exploiter ces difficultés?

\section{Les difficultés identitaires du chercheur}

Au cours de la recherche, à l'arrivée sur le terrain à Bissighin ou à WNB, malgré mes efforts de sobriété et de discrétion sur le plan matériel (vêtements sobres, véhicule garé discrètement loin du site d'enquête), malgré mes gestes de familiarité et de politesse sur le plan relationnel (salutations systématiques, discussions en langue locale mòoré), les habitants m'ont tout de suite perçu comme un étranger au statut social spécifique. Pas un étranger au sens de la nationalité, puisqu'ils ne doutaient pas que j'étais burkinabè, mais dans le sens simmelien de "“forme sociologique", caractérisée par le fait d'être à la fois fixé en un point de l'espace et détaché de ce même point» (Rammstedt, 1994: 147). Les habitants de Bissighin m’ont attribué donc, voire m'ont imposé cette caractéristique d'étranger. Je portais également cette «figure flottante» de l'étranger (Chaouite, 1997: 2-3), qui oscillait non seulement entre proximité et distanciation vis-à-vis des enquêtés, mais aussi entre la figure concrète et la figure construite de ma personne par mes interlocuteurs. La plupart de mes interlocuteurs étaient donc convaincus que je pouvais leur apporter une aide matérielle tout de suite, ou que j'étais en mesure de convaincre les autorités publiques d'améliorer leur cadre de vie.

À Bissighin, où j’ai travaillé en 2011 en tant que professionnel au sein d'une équipe d'experts, certains habitants m'assimilaient déjà à mes commanditaires et s'adressaient à moi comme si j'avais le pouvoir de satisfaire directement leurs doléances. Par exemple, en faisant référence à la nécessité du lotissement de sa zone, un habitant déclare: "Vous pouvez le faire... si vous le voulez.» En 2011, le chef de WNB m'a surnommé Sor Naba, ce qui signifie "chef de la voirie». Cette forme d'intronisation exprimait, d'une part, son appropriation du 
PHOTO 10.1

\section{Vue sur le paysage urbain du quartier non loti de Watinoma}

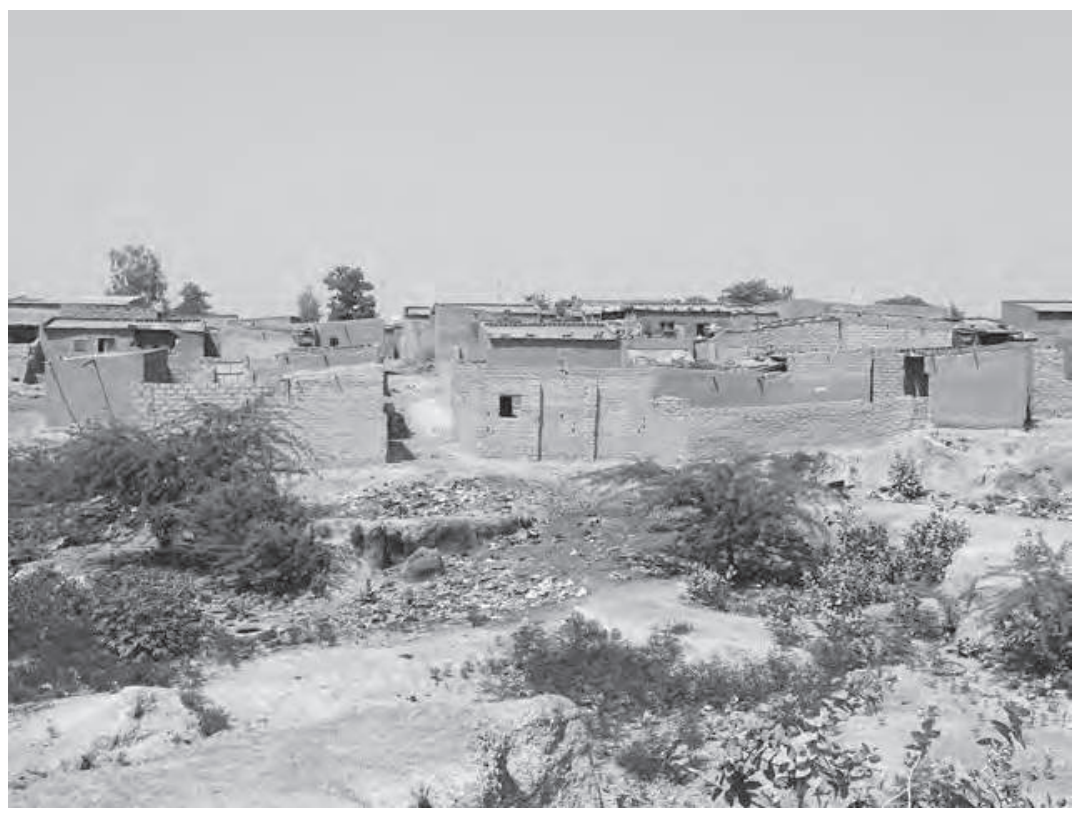

Photo: Léandre Guigma, 2016

projet et son désir de bénéficier d'un nouvel aménagement de voirie dans son quartier, et d'autre part, sa confiance en l'étude et en la personne que je suis pour y parvenir. À plusieurs reprises, le chef m'a également considéré comme un intermédiaire chargé de transmettre les doléances des habitants et de plaider en leur faveur auprès du ministère de l'Habitat et de l'Urbanisme et d'ONU-Habitat. La posture a donc évolué de chercheur-passeur, qui informe sur les actions du programme, à celle de chercheur-médiateur, qui représente un intermédiaire entre les habitants et leurs gouvernants.

J'ai fait face à trois formes d'identité, au sens de construit social et de relation que l'on construit avec son environnement. Tout d'abord, mon identité créée de représentant direct de l'autorité publique ou des bailleurs de fonds, vue par certains habitants des zones non loties. 
Ensuite, mon identité subie d'intermédiaire, en lien avec mon intervention dans le quartier en 2011, que d'autres habitants m'ont rappelée. Enfin, mon identité de chercheur, celle que je revendiquais, même si ses contours, son intérêt et sa finalité ne semblent ni pertinents ni saisissables dans ce contexte. Le discours langagier a joué ici un rôle essentiel dans la construction identitaire. À ce propos, Gervais-Lambony affirme que l'identité «correspond non pas à une réalité donnée mais à un discours qui propose un "ordre des choses" (2004: 470).

Ces identités multiples se révèlent dans le contexte d'une configuration développementiste, définie par Olivier de Sardan comme un «ensemble complexe d'institutions, de flux et d'acteurs, pour qui le développement constitue une ressource, un métier, un marché, un enjeu, ou une stratégie» (2001: 731). Doit-on rejeter ou assumer ces identités créées et subies?

Le problème d'identification s'est également posé à moi en matière de posture et de déconstruction de la pensée, donc par rapport à mon implication personnelle dans la recherche. Parmi ce qu'il qualifie de «risques du métier», Cefaï se demande «comment assurer la transition de la posture d'enquêteur et d'analyste à celle d'expert en maintenant une fidélité due aux enquêtés et une loyauté à ses propres convictions» (2002: 7). Dans le cas précis de cette recherche, la question a été inversée. À l'issue des observations et des enquêtes de terrain que l'équipe d'experts a menées, j'ai établi un diagnostic du cadre de vie et formulé des projets visant l'amélioration des sites étudiés. Ce document-projet, qu'ont apprécié les acteurs nationaux et internationaux (atelier national, ONU-Habitat, Union européenne) après plusieurs évaluations, a été présenté au Forum urbain mondial à Naples en juin 2012. Tout en assumant ces résultats de l'étude, j’ai été dans l'obligation de m'en défaire, de les remettre en question, de les déconstruire afin de rechercher auprès des acteurs de nouvelles tendances ou pratiques qui pourront confirmer, nuancer ou infirmer ces résultats d'étape.

Par ailleurs, j'ai dû faire face au fait que les promesses d'ONUHabitat et du ministère burkinabè de l'Habitat et de l'Urbanisme, prises 
РНOTO 10.2

\section{Point d'eau collectif (forage) de Bissighin}

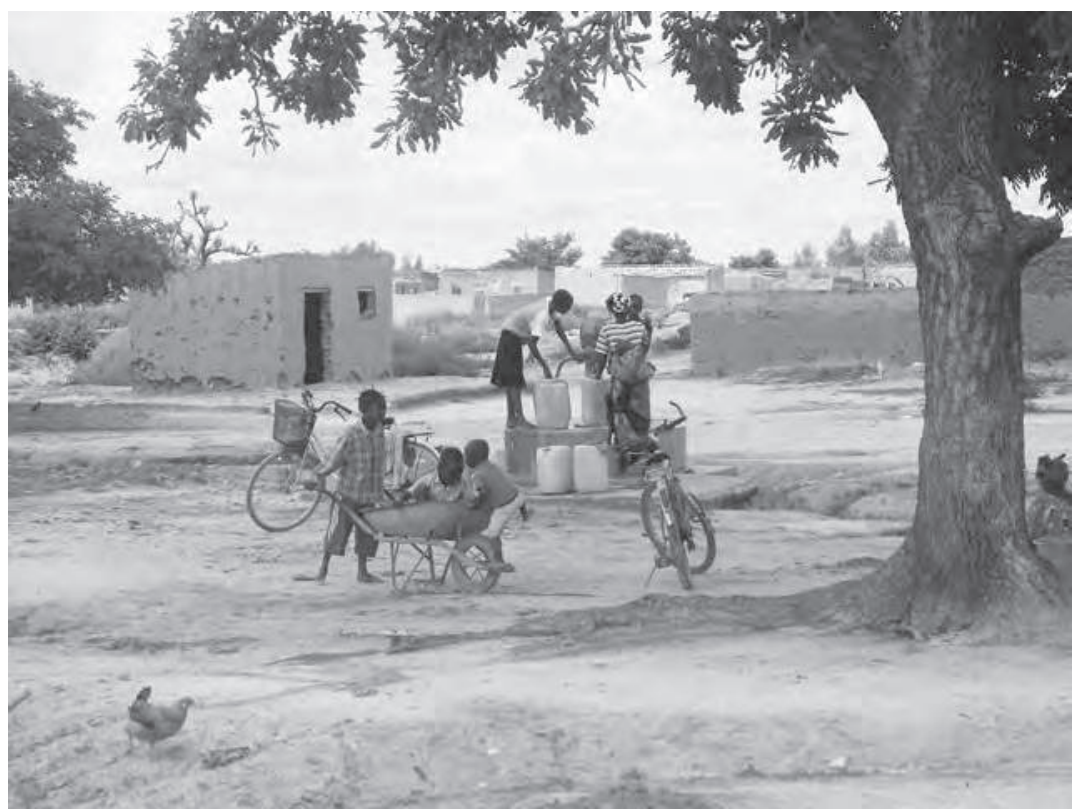

Photo: Léandre Guigma, 2011

en ma présence en tant qu'expert devant les habitants, n'étaient pas encore exécutées. Cette situation du terrain était à la fois intéressante et embarrassante. Elle constituait une condition propice où l'information en tant qu'objet d'analyse pouvait être investiguée sous la forme d'un canal de transmission produisant des effets induits: déformations de l'information, pratiques d'anticipation, accélération du processus de transformation ou relative passivité. Mais elle pouvait être embarrassante dans le sens où certains habitants n'hésitaient pas à exprimer sur ma personne leur colère, justifiée par les promesses non tenues de l'administration. En conséquence, les habitants ont adapté leurs discours et l'état de leur situation socioéconomique dans l'espoir de bénéficier d'aides diverses des autorités institutionnelles pour aménager leur quartier ou pour construire eux-mêmes leurs logements. 
En effet, attendant l'exécution des projets d'aménagement dans leur quartier, les habitants fournissaient des informations selon la nature du projet qu'ils souhaitaient voir se réaliser. Peut-on considérer cette fonction d'intermédiaire que j'assurais vis-à-vis de l'administration publique et des bailleurs comme un contre-don à assumer, en échange des informations obtenues auprès des habitants? Cette question pose plus largement celle du rapport entre le chercheur et son terrain. Le chercheur travaille-t-il sur, avec ou pour son terrain? Le chercheur doit-il tenir une position d'extériorité par rapport à celui-ci?

\section{Contourner les embûches du terrain}

À cette double identité d'expert et de chercheur s'est greffée, quelquefois, celle d'intermédiaire entre les populations résidentes des zones non loties et les autorités publiques, voire celle d'allié de l'un ou l'autre groupe d'acteurs qui n'hésitaient pas à me faire des confidences ou à solliciter de ma part une intermédiation. Ces identités multiples sontelles un atout ou une contrainte?

La prise de conscience par le chercheur de ses multiples identités serait une première étape de la démarche. La deuxième étape consisterait à cerner, dans la situation précise de l'entretien, laquelle des identités du chercheur est perçue par l'interlocuteur, notamment afin de pouvoir lier les réponses de l'enquêté à cette identité spécifique.

Lors de la retranscription ou de la lecture des entretiens, j'ai pu me rendre compte clairement de mes identités créées et subies. D’autres attitudes, gestes ou émotions (in)exprimées ont également influencé les réponses de mes interlocuteurs. Est-il authentique et utile de garder devant son interlocuteur une attitude de distanciation, permettant au chercheur de garantir son objectivité d'étranger et de ne pas virer indigène?

Dans certains cas, la posture de représentant direct de l'autorité publique ou du bailleur de fonds m'a permis d'obtenir de la part de mes interlocuteurs une ouverture et une confiance qui m’ont aidé à 
mieux cerner leurs besoins. À maintes reprises, j’ai pu provoquer, par mon attitude, non pas de la curiosité ou de l'indifférence, mais une certaine empathie qui a contribué à mettre mes interlocuteurs en confiance et à leur délier la langue. En évoquant la gestion de la relation aux enquêtés, Cefaï remarque que «l'enquêteur trouve parfois son salut dans le maintien d'une ambiguïté qui lui ouvre des portes sans pour autant révéler ses positions personnelles» (2002: 6).

Le décryptage des transformations du terrain semble exiger de la part du chercheur à la fois une proximité au terrain, afin d'observer et cerner ces évolutions, et une distance nécessaire, pour découvrir et analyser les causes profondes et les circonstances quelquefois inavouées de ces mutations. Comment concilier ces deux rapports au terrain, a priori contradictoires?

Sans renier tous les avantages de la confiance et de la proximité avec nos interlocuteurs de Bissighin et de $\mathrm{WNB}$, on peut reconnaître les limites de cette approche exclusive qui ne permet pas de décrypter toutes les logiques et les stratégies des personnes enquêtées. Pour m’en défaire, j’ai expérimenté d'autres rapports au terrain. Il s'agit de la mise en place d'enquêtes à passages répétés et de formes d'observation ou d'entretiens indirects, en évitant d'être physiquement sur le terrain et en utilisant d'autres canaux d'information. J'ai pu suivre les réactions des habitants auprès d'autres interlocuteurs grâce à la presse, aux commentaires et aux comptes-rendus d'entretiens menés par des tiers, ainsi que par ma participation en tant qu'observateur à des ateliers et à des réunions administratives. Ces astuces méthodologiques ont permis de révéler d'autres réalités, différentes ou complémentaires des résultats issus des premières approches de proximité.

\section{Les leçons à tirer}

Ma distanciation du terrain et mes observations indirectes ont permis de découvrir différents registres de discours des autorités publiques, parfois contradictoires en fonction des interlocuteurs, selon qu'elles s'adressent aux bailleurs de fonds ou aux populations. Il en est de 
même pour les discours des populations locales qui se contredisent selon qu'ils sont adressés aux bailleurs de fonds internationaux ou aux autorités publiques nationales.

J'ai adjoint un discours qui exprime le point de vue des acteurs sur le terrain, produit par mes propres réflexions et analyses. Ce discours n'est guère une synthèse des discours entendus, mais le fruit d'un parti pris fondé à la fois sur l'objectivité et la sincérité du chercheur.

Le chercheur s'engage donc dans sa propre recherche. Plus qu'un informateur ou un enquêteur, plus qu'un médiateur ou un simple intermédiaire, le chercheur est coauteur et coproducteur d'un discours sur l'objet de sa recherche. Mais ce discours restera empreint d'une subjectivité par ailleurs indispensable à la remise en question permanente de la recherche, plus particulièrement dans le domaine des sciences sociales.

Il semble que l'identité du chercheur et son rapport au terrain ne soient pas dictés seulement par sa propre conviction: ils sont aussi, et parfois surtout, influencés par les représentations de ses interlocuteurs. Les relations humaines que le chercheur entretient avec ses interlocuteurs peuvent engendrer une certaine familiarité, voire une ambiguité face aux conséquences majeures pour la présupposée et généralement admise objectivité de la recherche. C'est pourquoi garder une distance par rapport au terrain apparaît indispensable, notamment par la gestion des temps d'enquête et par l'exploitation de moyens indirects pour compléter, nuancer, conforter ou infirmer les informations.

La gestion du temps remet en question la pertinence d'observer des faits en temps réel dans une démarche d'observation participante prolongée, ou de se distancier du terrain en décrivant des situations conjoncturelles isolées en référence à une situation initiale éloignée, au risque de perdre de vue les transformations quotidiennes en matière d'allers-retours et de situations d'entre-deux.

L'articulation nécessaire du rapport entre le chercheur et son terrain marque la subtilité de la participation observante en tant que méthode d'enquête. Ce sont donc à la fois les dires (et les non-dits) 
des acteurs, l'observation ethnographique et celle du terrain qui font l'objet d'une réobjectivation régulière tout au long du processus de construction de la recherche. En ce sens, le choix d'un terrain d'enquête est «la subjectivité d'une procédure d'objectivation» (Raoul, 2002). 



\section{Les auteurs}

Mauricio Anaya est architecte, docteur en urbanisme et chercheur invité à l'Institut de recherche de la Faculté d'architecture et sciences de l'habitat de l'Université Majeur de San Simón. Il a travaillé comme consultant en planification pour la municipalité de Cochabamba.

Pauline Bosredon est maître de conférences en géographie et aménagement à l'Université Lille 1. Ses recherches portent sur les mutations urbaines contemporaines vues à travers le prisme des processus et des politiques de patrimonialisation et de développement des activités culturelles. Sa réflexion porte sur l'accès aux ressources de la ville pour tous dans différents contextes non occidentaux - en Éthiopie, dans les Territoires palestiniens occupés et au Brésil.

Valeria Cartes Leal est architecte-urbaniste et docteure en Art de bâtir et Urbanisme et Sciences de l'ingénieur de l'Université catholique de Louvain. Elle est chargée de mission du Service École au Bureau bruxellois de la planification - Région de Bruxelles-Capitale. Ses recherches portent sur l'école au cœur de projets de société et de l'espace public.

Bianca De Marchi Moyano est communicatrice sociale et master en gestion du patrimoine et développement territorial de l'Université Mayor de San Simón (Bolivie). Elle est docteure en Art de bâtir et Urbanisme de l'Université Catholique de Louvain (Belgique) et chercheuse responsable de la ligne «théorie et histoire» du Centre de recherche sociale de la Vice-présidence de l'État plurinational à la Paz. 
Gabriel Fauveaud a obtenu son doctorat en géographie à l'Université de Paris 1 Panthéon-Sorbonne en 2013. Il est chercheur invité au Centre d'études et de recherches international de l'Université de Montréal (CÉRIUM) et chercheur associé à l'UMR 8586 Prodig.

Léandre Guigma est gérant de l'agence PERSPECTIVE d'architecture et d'urbanisme sise à Ouagadougou. Il est doctorant en études urbaines à l'Université Paris 8 Vincennes Saint-Denis. Ses travaux de recherche et son expertise portent sur l'aménagement des quartiers populaires dits «non-lotis» de Ouagadougou et de leurs acteurs.

Divya Leducq est maître de conférences en Aménagement de l'espace et Urbanisme à Polytech Tours. Ses travaux mettent en relief les spécificités des projets et l'évolution des dynamiques urbaines et régionales dans des contextes du Nord (Lille, Tours, Québec) et du Sud global (Inde, Hanoi, Océan Indien).

Cécilia Montoya Antich est professeur à la Universidad Peruana de Ciencias Aplicadas (UPC) à Lima. Doctorante en géographie à Paris 1, elle mène ses recherches sur les nouvelles formes de gouvernance, les espaces publics, la décentralisation et les espaces littoraux. Elle a enseigné à l'Université de Paris 13 et à la Universidad Nacional Mayor de San Marcos (UNMSM) à Lima. Elle est associée au laboratoire PRODIG (France).

Aniss M. Mezoued est architecte-urbaniste et docteur en Art de bâtir et Urbanisme. Il est chercheur à l'École Polytechnique fédérale de Lausanne et à l'Université catholique de Louvain. Ses recherches portent sur les liens entre mobilité, urbanisme et développement territorial. Il est l'un des auteurs du MOOC «Mobilité et Urbanisme» https://www.coursera.org/learn/mobiurba

Claire Simonneau est docteure en aménagement de l'Université de Montréal et postdoctorante au sein de la Faculté d'architecture, d'ingénierie architecturale, d'urbanisme de l'Université Catholique de Louvain. Ses intérêts de recherche se situent au croisement de l'aide internationale et de l'urbanisme.

Issa Sory est enseignant-chercheur au Département de géographie de l'Université de Koudougou. Il est membre du laboratoire Groupe de recherche sur les initiatives locales (GRIL). Ses recherches portent sur les politiques urbaines, l’aménagement et les services urbains au Burkina Faso. 


\section{Bibliographie}

Alexander, Christopher, «A pattern language», dans Christopher Alexander et al. (dir.), A pattern language: Towns, buildings, constructions, New York, Oxford University Press, 1977, p. ix-xix.

Amin, Ash et Stephen Grahama, "The ordinary City», Transactions of Institute of British Geographers, vol. 22, n 4 , 1997, p. 411-429.

Amin, Samir et al., "Qu'est-ce que le néolibéralisme?», Actuel Marx, n 40, 2006, p. 12-23.

Anaya, Mauricio, Prácticas cotidianas y estrategias producción de continuidad urbana en la ciudad de Cochabamba, Louvain-la-Neuve, Presses universitaires de Louvain, 2013.

Anderson, Ben et Colin McFarlane, «Assemblage and Geography», Area, vol. 43, $\mathrm{n}^{\circ}$ 2, 2011, p. 124-127.

Arborio, Anne-Marie et Pierre Fournier, L'observation directe, Paris, Armand Colin, 2008.

AtKinson, Adrian, "Environnement urbain et développement: le cas de l'Asie du Sud-Est», dans Françoise Lieberherr-Gardiol et Germán Solinis (dir.), Quelles villes pour le $21^{e}$ siècle?, Suisse, Les éditions Infolio, 2012, p. 264-310.

Bacqué, Marie-Hélène et Mario Gauthier, "Participation, urbanisme et études urbaines », Participations, n ${ }^{\circ}$ 1, 2010, p. 36-66.

Bako-Arifari, Nassirou et Pierre-Yves Le Meur, «Une anthropologie sociale des dispositifs de développement» dans Jean-François Baré (dir.), L'évaluation de politiques de développement. Approches pluridisciplinaires, Paris, L'Harmattan, 2001, p. 121-173.

Baron, Catherine et Clément Frenoux, «Ville néolibérale et accès à l'eau au Sud: changer ou aménager le modèle? », dans Lise Bourdeau-Lepage (dir.), Regards sur la ville, Paris, Economica, 2012, p. 111-142.

BARon, Catherine et Élisabeth Peyroux, "Services urbains et néolibéralisme: Approches théoriques et enjeux de développement: Regards croisés sur deux terrains contrastés (Burkina Faso, Afrique du Sud)», Cahiers d'études africaines, $\mathrm{n}^{\mathrm{o}}$ 202-203, 2011, p. 369-393. 
BAron, Myriam et Laurent JÉGou, «Carte à la une: le monde selon... le Web of Science», Géoconfluences, 2016, <bit.ly/2nLTTRw >.

Barthel, Pierre-Arnaud et Éric Verdeil, «Experts embarqués dans le «tournant financier»: Des grands projets urbains au sud de la Méditerranée », Les Annales de la recherche urbaine, $\mathrm{n}^{\circ} 104,2008$, p. 38-48.

BARThel, Pierre-Arnaud, Tunis en projet(s): la fabrique d'une métropole au bord de l'eau, Presses universitaires de Rennes, 2006.

Bastien, Soulé, «Observation participante ou participation observante? Usages et justifications de la notion de participation observante en sciences sociales", Recherches qualitatives, vol. 27, $\mathrm{n}^{\circ}$ 1, 2007, p. 127-140.

Beard, Victoria, "Learning Radical Planning: The Power of Collective Action", Planning Theory, vol. 2, $\mathrm{n}^{\circ}$ 1, 2003, p. 13-35.

Beaudet, Gérard, Meloche, Jean-Philippe et Franck Scherrer, Questions d'urbanisme, Presses de l'Université de Montréal, 2012.

Becker, Howard, Les ficelles du métier, Paris, La Découverte, 2002.

Bellina, Séverine, Magro, Hervé et Violaine De Villmeur (dir.), La gouvernance démocratique: un nouveau paradigme pour le développement, Paris, Karthala, 2008.

Bénit-Gbaffou, Claire, Fabiyi, Seyi et Élisabeth Peyroux (dir.), Sécurisation des quartiers et gouvernance locale: Enjeux et défis pour les villes africaines (Afrique du Sud, Kenya, Mozambique, Namibie, Nigeria), Paris, Karthala, 2009.

Benjamin, Solomon, «Occupancy Urbanism: Radicalizing Politics and Economy Beyond Policy and Programs", International Journal of Urban and Regional Research, vol. 3, nº 32, 2008, p. 719-729.

Berque, Augustin, La Pensée paysagère, Paris, Archibooks, 2008.

Berry-Chikhaoui, Isabelle, «Les notions de citadinité et d'urbanité dans l'analyse des villes du monde arabe: Essai de clarification", Les Cahiers d'EMAM. Études sur le monde arabe et la Méditerranée, $\mathrm{n}^{\circ}$ 18, 2009, p. 9-20.

Bertrand, Georges, «Le paysage entre la Nature et la Société», dans Alain Roger (dir.), La théorie du paysage en France. 1974-1994, Seyssel, Champ Vallon, 1995, p. 88-108.

Bertrand, Monique, «Du logement à la ville: nouvel agenda urbain et questionnements scientifiques", Autrepart, ${ }^{\circ}$ 25, 2003, p. 5-19.

Bertrand, Monique, «Mobilité, pauvretés: les villes interrogées - Introduction », Tiers Monde, $\mathrm{n}^{\circ}$ 201, 2010, p. 7-23.

Bertrand, Monique, "Villes africaines, modernités en question", Tiers Monde, vol. $39, \mathrm{n}^{\circ} 156,1998$, p. 885-904.

Bertrand, Monique, La question foncière dans les villes du Mali: marchés et patrimoines, Paris, Karthala, 1994.

Bidou-Zachariasen, Catherine (dir.), Retours en ville - des processus de "gentrification» urbaine aux politiques de «revitalisation» des centres, Paris, Descartes \& Cie, 2003.

Blanchet, Anne et Alain Gotman, L'entretien, Paris, Armand Colin, 2007. 
BLOt, Julie et Amandine SpIRE, «Déguerpissements et conflits autour des légitimités citadines dans les villes du Sud», L'Espace Politique, $\mathrm{n}^{\circ}$ 22, 2014, <espacepolitique. revues.org/2893>.

Bondaz, Julien, IsNart, Cyril et Anaïs LEBLON, «Au-delà du consensus patrimonial», Civilisations, vol. 61, $\mathrm{n}^{\circ} 1,2012$, p. 9-22.

Bonvalet, Catherine et al., Recompositions du savoir urbain entre nord et sud, Paris, Orstom, 1997.

Bonvalet, Catherine et Françoise Dureau, «Les modes d'habiter: des choix sous contraintes", dans Françoise Dureau et al. (dir.), Métropoles en mouvement. Une comparaison internationale, Paris, Anthropos, 2000, p. 131-153.

Bosredon, Pauline et Julie Dumas, «Régulations et contestation du droit: la production des espaces urbains en question», Géocarrefour, vol. 88, nº 3, 2013, <geocarrefour.revues.org/9199>.

BosRedon, Pauline, «Le processus patrimonial à Hébron: Accaparement ou partage d'une centralité urbaine disputée? », dans Yves Bonny, Nicolas BaUtès et Vincent GoüsET, L'espace en partage: Approche interdisciplinaire de la dimension spatiale des rapports sociaux, Presses Universitaires de Rennes, "Géographie sociale», 2016.

Bosredon, Pauline, Gravereau, Sophie, Gregoris, Marie-Thérèse et Anissa HABANE, "Art et culture à Ramallah dans les Territoires palestiniens occupés (TPO): Entre patrimoine, revendications politiques et développement territorial», Belgeo, vol. 3, 2014, <belgeo.revues.org/13392>.

Brenner, Neil et Nik Theodore, "Cities and the Geographies of "Actually Existing Neoliberalism" ", Antipode, vol. 34, n 3, 2002, p. 349-379.

BrICKELL, Katherine, "The Whole World Is Watching”: Intimate Geopolitics of Forced Eviction and Women's Activism in Cambodia", Annals of the Association of American Geographers, vol. 104, n $\mathrm{n}^{\mathrm{0}}$ 6, 2014, p. 1256-1272.

Brickell, Katherine et Ayona Datta (dir.), Translocal Geographies, Routledge, 2011.

BUlle, Sylvaine, «Entre monde et patrie: l'entreprise patrimoniale palestinienne, 1995-2002", Autrepart, nº33, janvier 2005, p. 127-140.

CAIllé, Alain et Stéphane Duforx, Le tournant global des sciences sociales, Paris, La Découverte, 2013.

CAPron, Guénola, Quand la ville se ferme: Quartiers résidentiels sécurisés, Paris, Bréal, 2006.

CARTEs LeAL, Valeria, L'école, l'enfant et la ville: les conditions de l'urbanisme scolaire: cas de la Région de Bruxelles-Capitale, Presse universitaires de Louvain, Université Catholique de Louvain, 2015.

Castells, Manuel, L'ère de l'information 1, La société en réseaux, Paris, Fayard, 1999.

CAvin, Joëlle Salomon, «Éloge de la concentration urbaine. Plaidoyer de la Banque mondiale en faveur de la grande ville », Cybergeo: European Journal of Geography, 2009, <cybergeo.revues.org/22685>.

Cefä̈, Daniel, «Éditorial», Cultures \& Conflits, 2003, <http://conflits.revues.org >.

Cefä̈, Daniel (dir.), L'enquête de terrain, Paris, La Découverte/M.A.U.S.S., 2003. 
Chakrabarty, Dipesh, Provincializing Europe: Postcolonial Thought and Historical Difference, Princeton University Press, 2000.

ChaléARD, Jean-Louis (dir.), Métropoles aux Suds, le défi des périphéries?, Paris, Karthala, 2014.

ChalÉArd, Jean-Louis et Alain Dubresson (dir.), Villes et campagnes dans les pays du Sud: géographie des relations, Paris, Karthala, 1999.

Chaléard, Jean-Louis et Roland Pourtier (dir.), Politiques et dynamiques territoriales dans les pays $d u$ Sud, Paris, Publications de la Sorbonne, 2000.

Chant, Sylvia, "Cities through a "gender lens": a golden "urban age" for women in the global South?», Environment and Urbanization, vol. 25, nº 1, 2013, p. 9-29.

Chaouite, Abdellatif, «L'étranger, une figure flottante», Ecarts d'identité, n 81 , juin 1997, p. 2-3.

Chenal, Jérôme et al., Quelques rues d'Afrique: Observation et gestion de l'espace public à Abidjan, Dakar et Nouakchott, Lausanne, LaSUR, 2009.

Chenal, Jérôme et al., «Esquisse d'une théorie «alter-moderne» de la ville africaine ", EspacesTemps.net, 2009.

Chibber, Vivek, Postcolonial theory and the specter of capital, Verso, New York, 2013.

Choplin, Armelle, "Le foncier urbain en Afrique: entre informel et rationnel, l'exemple de Nouakchott (Mauritanie) », Annales de géographie, vol. 1, n 647, 2006, p. 69-91.

Choplin, Armelle, "Désoccidentaliser la pensée urbaine", Métropoliques.eu, 2012 http://www.metropolitiques.eu/Desoccidentaliser-la-pensee.html.

Collectif Inverses, Morelle, Marie, Jacquot, Sébastien, Tadié, Jérôme, Bautès, Nicolas, Bènit-Gbaffou, Claire, Maccaglia, Fabrizio, Rivelois, Jean et Alexis SIERRA, "L'informalité politique en ville. 8 chercheurs et 9 villes face aux modes de gouvernement urbain ", L'Espace Politique, vol. 29, nº 2, 2016, <espacepolitique. revues.org/3806>.

Comeliau, Christian, «Développement du "développement durable” ou blocages conceptuels?», Tiers Monde, $\mathrm{n}^{\circ}$ 35, 1994, p. 61-76.

Corboz, André, Le territoire comme palimpseste et autres essais, Paris, Éditions de l'Imprimeur, 2001.

Costes, Laurence, «Le Droit à la ville de Henri Lefebvre: quel héritage politique et scientifique? ", Espaces et sociétés, nº 140-141, 2010, p. 177-191.

Cresswell, Tim et Peter Merriman, Geographies of Mobilities: Practices, Spaces, Subjects, Routledge, 2011.

Cusset, François, French theory: Foucault, Derrida, Deleuze et Cie et les mutations de la vie intellectuelle aux États-Unis, Paris, La Découverte, 2013.

Daina-Gueida, Laurence, Circulation des savoirs et reconfiguration des idées, Perspectives croisées: France-Brésil, Lille, Presses universitaires du Septentrion, 2016.

Dansereau, Francine et Françoise Navez-Bouchanine (dir.), Gestion du développement urbain et stratégies résidentielles des habitants, Paris, L'Harmattan, 2002.

Darbon, Dominique et Ivan Crouzel, «Administrations publiques et politiques publiques des Afriques», dans Mamoudou Gazibo et Céline Thiriot (dir.), Le 
politique en Afrique: État des débats et pistes de recherche, Paris, Karthala, 2009, p. 71-101.

DARBon, Dominique, «À qui profite le mime? Le mimétisme institutionnel confronté à ses représentations en Afrique», dans Yves MÉNY (dir.), Les politiques du mimétisme institutionnel: La greffe et le rejet, Paris, L'Harmattan, 1993, p. 113-137.

Darbon, Dominique (dir.), La politique des modèles en Afrique: Simulation, dépolitisation et appropriation, Paris, Karthala, 2009.

Davis, Mike, Planet of Slums, Londres, Verso, 2006.

De Certeau, Michel, L'invention du quotidien: Arts de faire (vol. 1), Paris, Gallimard, 1990.

DeClÈve, Bernard, "Mutations territoriales et dimension culturelle de la conduite des projets de renouveau urbain", Savoirs et jeux d'acteurs pour des développements durables, Academia-Bruylant, 2001, p. 501-522.

Declève, Bernard, "Coproduire une puissance publique d'intervention", dans Bernard Declève et al. (dir.), "Coproduire nos espaces publics: Formation-actionrecherche», Presses universitaires de Louvain, 2002, p. 69-83.

DeClève, Bernard, "Comment décrire une ville ordinaire?», préface à la thèse de Mauricio Anaya, Prácticas cotidianas y estrategias producción de continuidad urbana en la ciudad de Cochabamba, Louvain-la-Neuve, Presses universitaires de Louvain, 2013, p. xi-xvii.

Delcourt, Laurent, «Explosion urbaine et mondialisation: points de vue du sud», Alternatives Sud, vol. 14, $\mathrm{n}^{\circ}$ 2, 2007, <bit.ly/2orgk4f $>$.

Deler, Jean-Paul, LE BRIS, Émile et Graciela SCHNEIER (dir.), Les métropoles $d u$ Sud au risque de la culture planétaire, Paris, Karthala, 1998.

Denef, Julie, Nouvelles formes de médiation urbanistique et esthétique de l'ouverture, Louvain-la-Neuve, Presses universitaires de Louvain, 2011.

Denis, Éric (dir.), Villes et urbanisation des provinces égyptiennes: vers l'écoumènopolis, Paris, Karthala, 2007.

Denis, Éric, "Qualifier les aires urbaines en forte expansion dans les Suds: Positionnement au prisme de travaux sur l'expansion des villes en Asie ", L'Espace géographique, vol. 44, nº 4 , 2015, p. 307-324.

De Rijck, Kim et Jean-Philippe Peemans, Mobilité et paysage. Les rapports entre espaces urbains, périurbains et ruraux: convergences et divergences des regards $d u$ développement et de l'urbanisme, Presses universitaires de Louvain, 2004.

Devisme, Laurent, «Le praticien réflexif et le théoricien activiste», Urbanisme, $\mathrm{n}^{\circ} 372$, 2010, p. 41-43.

Di Meo, Guy, «Patrimoine et territoire, une parenté conceptuelle», Espaces et Sociétés, $\mathrm{n}^{\circ} 78,1995, \mathrm{p} .16-33$.

Dick, Howard W. et Peter J. Rimmer, The city in Southeast Asia: Patterns, processes and policy, NUS Press, 2009.

DoraIs, Louis-Jacques, "La construction de l'identité», Actes: Culture française d'Amérique, 2004, p. 1-11. 
Dorier-Apprill, Élisabeth et Cécilia Meynet, «Les ONG: acteurs d'une "gestion disputée" des services de base dans les villes africaines? ", Autrepart, vol. 3, nº 35, 2005, p. 19-37.

Dorier-Apprill Élisabeth et Sylvy Jaglin, «Introduction. Gestions urbaines en mutation: du modèle aux arrangements locaux", Autrepart, $\mathrm{n}^{\circ}$ 21, 2002, p. 5-15.

Dorier-Apprill, Élisabeth et Philippe Gervais-Lambony (dir.), Vies citadines, Paris, Belin, 2007.

Douglass, Mike et John Friedmann, Cities for Citizens: Planning and the Rise of Civil Society in a Global Age, Wiley \& Son, 1998.

Dupont Véronique, «Création de nomades urbains et appauvrissement. Impact des politiques d'éradication des camps de squatters à Delhi", Tiers Monde, $\mathrm{n}^{\circ}$ 201, 2010, p. 25-45.

Dupont, Véronique et Djallal Gérard Heuzé (dir.), La ville en Asie du Sud: analyse et mise en perspective, Paris, Éditions de l'École des hautes études en sciences sociales, 2007.

DURAND-LASSERVE, Alain, «Réguler les marchés fonciers pour des villes inclusives», dans Pierre JaCQuet, Rajendra K. PAChauri et Laurence Tubiana, Regards sur la Terre 2010: Villes, changer de trajectoire, Paris, Presses de Sciences Po, 2010.

Durand-Lasserve, Alain, «La question foncière dans les villes du Tiers-monde: un bilan", Économies et sociétés, vol. 38, nº 7, 2004, p. 1183-1211.

DurEau, Françoise et al., (dir.), Villes et sociétés en mutation. Lectures croisées sur la Colombie, Paris, Anthropos, 2003.

Dureau, Françoise et Jean-Pierre LÉvy, «Villes et mobilités au Nord et au Sud: la construction d'une problématique commune», Autrepart, n 41, 2007, p. 135-148.

Edensor, Tim et Jayne Mark (dir.), Urban Theory Beyond the West: A World of Cities, Routledge, 2012.

Fainstein, Susan S., "New Directions in Planning Theory», Urban Affairs Review, vol. $35, \mathrm{n}^{\circ} 4$, 2000, p. 451-478.

Fainstein, Susan S., "Planning Theory and the City», Journal of Planning Education and Research, vol. 25, 2005, p. 121-130.

FARIAs, Ignacio et Thomas Bender (dir.), Urban Assemblages: How Actor-Network Theory Changes Urban Studies, Routledge, 2010.

Fauveaud, Gabriel, La production des espaces urbains à Phnom Penh: pour une géographie sociale de l'immobilier, Paris, Publications de la Sorbonne, 2015.

Forester, John, "Planning in the Face of Power", Journal of the American Planning Association, vol. 48, 1982, p. 67-80.

Franck, Manuelle et Thierry Sanjuan (dir.), Territoires de l'urbain en Asie. Une nouvelle modernité?, Paris, CNRS Éditions, 2015.

Franck, Manuelle, Goldblum, Charles et Christian Taillard (dir.), Territoires de l'urbain en Asie du Sud-Est: métropolisations en mode mineur, Paris, CNRS éditions, 2012.

Friedmann, John, «The world city hypothesis», Development and Change, vol. 17, $\mathrm{n}^{\mathrm{o}} 1,1986$, p. 69-83. 
Friedmann, John, Insurgencies: Essays in Planning Theory, Routledge, 2011.

Friedmann, John, Planning in the Public Domain: From Knowledge to Action, Princeton University Press, 1987.

Gehl, Jan et Birgitte Svarre, How to Study Public Life, Island Press, 2013.

Gervais-Lambony, Philippe, "Mondialisation, métropolisation et changement urbain en Afrique du Sud», Vingtième Siècle, Revue d'histoire, vol. 1, n 81, 2004, p. 57-68.

Gervais-Lambony, Philippe et al. (dir.), La justice spatiale et la ville: regards du Sud, Paris, Karthala, 2014.

Gervais-Lambony, Philippe, «De l'usage de la notion d'identité en géographie. Réflexions à partir d'exemples sud-africains / The notion of identity in geography, a reflection through South African exemples", Annales de Géographie, t. 113, n 638639, 2004, p. 469-488.

Gervais-Lambony, Philippe, De Lomé à Harare: le fait citadin, Paris, Karthala, 1994.

Ghorra-Gobin, Cynthia et Alain Musset, «Par-delà le Nord et le Sud: débats sur la ville américaine», Cahiers des Amériques latines, nº 59, 2010, p. 11-13.

GHorra-Gobin, Cynthia, «Une ville mondiale est-elle forcément une ville globale? Un questionnement de la géographie française», L'Information géographique, vol. 71, $\mathrm{n}^{\circ} 2$ 2, 2007, p. 32-42.

Giraut, Frédéric, "Les ambiguïtés de la nouvelle doctrine spatiale de la Banque mondiale», Cybergeo: European Journal of Geography, 2009, <cybergeo.revues. org/22695>.

Glassman, Jim, «Primitive Accumulation, Accumulation by Dispossession, Accumulation by "Extra-Economic" Means", Progress in Human Geography, vol. 30, $\mathrm{n}^{\circ} 5,2006$, p. 608-625.

Gold, Raymond, "Jeux de rôles sur le terrain: Observation et participation dans l'enquête sociologique», dans Daniel Cefaï (dir.), L'Enquête de terrain, Paris, La Découverte, 2003, p. 340-349.

Goldblum, Charles, «Phnom Penh ou la métropolisation dépendante: entre processus d'urbanisation et stratégie d'aménagement», dans Manuelle FrancK, Charles Goldblum et Christian Taillard (dir.), Territoires de l'urbain en Asie du Sud-Est: Métropolisations en mode mineur, Paris, Éditions du CNRS, 2012, p. 25-73.

Goldblum, Charles, Osmont, Annick et Isabel Diaz (dir.), Gouverner les villes $d u$ Sud: Défis pour la recherche et pour lraction, Actes du Colloque International du Programme de recherche urbaine pour le développement, Paris, Unesco, 2004.

Goldblum, Charles, Métropoles de l'Asie du Sud-Est: stratégies urbaines et politiques du logement, Paris, L'Harmattan, 1988.

Granier, Gérard et Yvette Veyret (dir.), Développement durable: Quels enjeux géographiques?, La Documentation photographique, dossier n8053, 2006.

Granotier, Bernard, La planète des bidonvilles: perspectives de l'explosion urbaine dans le tiers monde, Paris, Seuil, 1980.

Gravari-BArbas, Maria (dir.), Habiter le patrimoine: enjeux, approches, vécu, Presses universitaires de Rennes, 2005. 
Gravari-Barbas, Maria et Vincent Veschambre, «Patrimoine: derrière l'idée de consensus, les enjeux d'appropriation de l'espace et des conflits», dans Patrice Melé, Corinne Larrue et Muriel Rosemberg (dir.), Conflits et territoires, Presses universitaires François-Rabelais, 2004, p. 67-82.

Gravari-BArbas, Maria, «le "sang” et le "sol”: le patrimoine, facteur d'appartenance à un territoire urbain", Géographie et culture, n² 20, 1996, p. 55-68.

Grosjean, Michèle et Jean-Paul Thibaud (dir.), L'espace urbain en méthode, Paris, Parenthèses, 2001.

Gugler Joseph (DIr.), World Cities Beyond the West-Globalization, Development and Inequality, Cambridge University Press, 2004.

Gumuchian, Henri et Christian Marois (dir.), Initiation à la recherche en géographie, aménagement, développement territorial, environnement, Montréal/Paris, Les Presses de l'Université de Montréal/Économica, 2000.

Habane, Anissa, Fabrique patrimoniale, culturelle et touristique dans un espace en tension: le cas des villes palestiniennes, Université Lille 1, 2015.

Habermas, Jürgen, L'espace public, Paris, Payot, 1978.

Hancock, Claire, « «Délivrez-nous de l'exotisme»: quelques réflexions sur des impensés de la recherche géographique sur les Suds (et les Nords) ", Autrepart, $\mathrm{n}^{\circ} 41,2007$, p. $69-81$.

HANCOCK, Claire, «Décoloniser les représentations: esquisse d'une géographie culturelle de nos "Autres»", Annales de géographie, $\mathrm{n}^{\circ}$ 660-661, 2008, p. 116-128.

Hansen, Karen Tranberg et al., Youth and the City in the Global South, Indiana University Press, 2008.

Hartman, Chester, Between Eminence and Notoriety: Four Decades of Radical Urban Planning, New Brunswick, CUPR Press, 2002.

Harvey, David, «Neoliberalism as Creative Destruction», The Annals of the American Academy of Political and Social Science, $\mathrm{n}^{\circ}$ 610, 2007, p. 21-44.

Harvey, David, A Brief History of Neoliberalism, Oxford University Press, 2005.

Healey, Patsy, «The Communicative Turn in Planning Theory and its Implications for Spatial Strategy Formation», Environment and Planning B: Planning and design, vol. 23, 1996, p. 217-234.

Healey, Patsy, Collaborative Planning: Shaping Places in Fragmented Societies, UBC Press, 1997.

Healey, Patsy, Urban Complexity and Spatial Strategies: Towards a Relational Planning for Our Times, Routledge, 2006.

Heinrichs, Dirk, Krellenberg Kerstin et Michail Fragkias, «Urban Responses to Climate Change: Theories and Governance Practice in Cities of the Global South", International Journal of Urban and Regional Research, vol. 37, $\mathrm{n}^{\circ} 6,2013$, p. $1865-1878$.

Herzog, Lawrence A., Global Suburbs: Urban Sprawl from the Rio Grande to Rio De Janeiro, Routledge, 2014.

Heurgon, Edith, "Retours sur la Prospective du présent», dans Edith Heurgon (dir.), Des «nous» et des «je» qui inventent la cité, Paris, Éditions de l'Aube, 2003. 
Hilgers, Mathieu et Jacinthe Mazzocchetti, «L'après-Zongo: entre ouverture politique et fermeture des possibles», Politique Afrique, $\mathrm{n}^{\circ}$ 101, 2006, p. 5-18.

Hubert, Michel, Paul Lewis et Michel Max Reynaud (dir.), Les grands projets urbains. Territoires, acteurs et stratégies, Presses de l'Université de Montréal, 2014.

IrazÁbal, Clara, Ordinary Places/Extraordinary Events: Citizenship, Democracy and Public Space in Latin America, Routledge, 2008.

Jacoвs, Jane M., «Urban Geographies I. Still Thinking Cities Relationally», Progress in Human Geography, vol. 36, $\mathrm{n}^{\circ}$ 3, 2012, p. 412-422.

JacoBs, Jane M., Edge of Empire: Postcolonialism and the City, Routledge, 2002.

JacoBs, Jane M., Déclin et survie des grandes villes américaines, Marseille, Parenthèses, 2012.

JAGLIN, Sylvy, «Villes disloquées? Ségrégations et fragmentation urbaine en Afrique australe», Annales de géographie, vol. 110, nº 619, 2001, p. 243-265.

Jenkins, Paul et al. (dir.), Planning and Housing in a Rapidly Urbanising World. New York, Routledge, 2007.

Josse, Guillaume et Zoé VAuQuelin, De la planification urbaine à la planification territoriale stratégique. Comment planifier les villes du Sud?, Paris, AFD, 2011.

Jouvenet, Morgan, «La culture du «bricolage» instrumental et l'organisation du travail scientifique enquête dans un centre de recherche en nanosciences ", Revue d'anthropologie des connaissances, vol. 1, $\mathrm{n}^{\circ}$ 2, 2007, p. 189-219.

Kaufmann, Jean-Claude, L'entretien compréhensif, Paris, Armand Colin, 2011.

Kessides, Christine, «The Urban Transition in Sub-Saharan Africa: Implications for Economic Growth and Poverty Reduction», The World Bank, Washington D.C., 2005.

Koop, Kirsten et Anne-Laure Amilhat, «Introduction», L'Information géographique, vol. 75, 2011, p. 6-14.

Lamizet, Bernard et Pascal Sanson, Les langages de la ville, Marseille, Parenthèses, 1997.

LAUMONIER, Laetitia, Le discours sur les villes en développement: épistémologie de la notion de ségrégation urbaine, Thèse de doctorat, Université de Paris 7 Diderot, 2004.

Laurent, Pierre-Joseph, «Le "big man” local ou la "gestion coup d'État” de l'espace public », Politique africaine, 2000, n 8o, p. 169-181.

LAUtier, Bruno, «Les limites de l'économie informelle comme alternative à la mondialisation libérale», Revue du MAUSS, vol. 1, n 21, 2003, p. 198-214.

LeducQ, Divya, Les systèmes territoriaux de l'innovation informatique dans l'Inde urbaine. Regards croisés depuis les villes de Pune, Thiruvananthapuram et Kochi, Université de Lille, thèse de doctorat en géographie et aménagement, 2011, p. 435 .

Lees, Loretta, "The Geography of Gentrification Thinking Through Comparative Urbanism ", Progress in Human Geography, vol. 36, n 2, 2012, p. 155-171.

Lees, Loretta, Hyun Bang Shin et Ernesto López-Morales (dir.), Global Gentrifications: Uneven Development and Displacement, Bristol, Policy Press, 2015. 
Lefebvre, Henri, «Le droit à la ville», L'Homme et la société, nº 6, 1967, p. 29-35.

Lefebvre, Henri, La révolution urbaine, Paris, Gallimard, 1970.

LÉvi-Strauss, Claude, La pensée sauvage, Paris, Plon, 1962.

LÉvy, Jacques, "Penser la ville: un impératif sous toutes les latitudes», Cahiers d'études sur la Méditerranée orientale et le monde turco-iranien, vol. 24, 1997, $<$ cemoti.revues.org/1458>.

LÉvy, Jacques, L'espace légitime: Sur la dimension géographique de la fonction politique, Paris, Presses de la Fondation nationale des sciences politiques, 1994.

LÉvy, Jacques, Le tournant géographique: penser l'espace pour lire le monde, Paris, Belin, 1999.

LoISEAU, Francine, "Évolution des rues à vivre dans quelques villes européennes", dans Centre d'études sur les réseaux, les transports, l'urbanisme et les constructions publiques (CERTU) (dir.), Conférence «Childstreet2005» Lyon, 2005.

LOMbARD, Jérôme et Benjamin STEск, «Quand le transport est d'abord un lieu!», Autrepart, $\mathrm{n}^{\circ}$ 32, 2004, p. 3-19.

Lorrain, Dominique (dir.), Métropoles XXL en pays émergents, Paris, Presses de Sciences Po, 2011.

Louiset, Odette, "Comment appréhender les slums indiens? Normes sociale et scientifique", L'Information géographique, vol. 75, $\mathrm{n}^{\circ}$ 1, 2011, p. 37-52.

LozA, Andrés, Permanencias y transformaciones territoriales, la construcción histórica del territorio en la micro región de los valles interandinos de Cochabamba, thèse en aménagement/urbanisme, Université Catholique de Louvain, 2008.

LUCAN, Jacques, «Où va la ville aujourd'hui? Formes urbaines et mixités», Éditions de la Villette, 2012.

LynCH, Kevin, The Image of the City, Harvard, MIT Press, 1960.

Magnaghi, Alberto, Le projet local, Torino, Mardaga, 2003.

Malaquais, Dominique, «Villes flux. Imaginaires de l'urbain en Afrique aujourd'hui», Politique Africaine, vol. 4, 2006, p. 15-37.

MAPPA, Sophia, «Rationalités et valeurs économiques au Nord et au Sud», dans Sophia MAPpa (dir.), La coopération internationale face au libéralisme, Paris, Karthala, p. 301-339.

Massey, Doreen, For Space, Londres, SAGE Publications, 2005.

Massiah, Gustave et Jean-François Tribillon, Villes en développement, Paris, La Découverte, 1988.

Mbembe, Achille et Sarah Nutall, «Writing the World from an African Metropolis», Public Culture, vol. 16, 2004. p. 347-372.

McCann, Eugene et Kevin Ward (dir.), Mobile Urbanism: Cities and Policymaking in the Global Age, University of Minnesota Press, 2011.

MCCANn, Eugene et Kevin WARD, «Relationality/Territoriality: Toward a Conceptualization of Cities in the World", Geoforum, vol. 41, n² 2, 2010, p. 175-184.

McFarlane, Colin, "The City as Assemblage: Dwelling and Urban Space», Environment and Planning-Part D, vol. 29, $\mathrm{n}^{\circ}$ 4, 2011, p. 649-671. 
McFarlane, Colin, «Rethinking Informality: Politics, Crisis, and the City», Planning Theory \& Practice, vol. 13, $\mathrm{n}^{\circ}$ 1, 2012, p. 89-108.

McFarlane, Colin, «Translocal Assemblages: Space, Power and Social Movements», Geoforum, vol. 40, n 4, 2009, p. 561-567.

McGee, Terry G. et Wang Yao-Lin, "La formation des mégapoles en Asie», Mappemonde, vol. 4, 1992, p. 2-3.

Melé, Patrice, "Conflits patrimoniaux et régulation urbaine», ESO Travaux et Documents, Rennes, Espaces et Sociétés - UMR 6590, 2005, p. 51-57.

MelÉ, Patrice, Patrimoine et action publique au centre des villes mexicaines, Presses Sorbonne Nouvelle, Institut des hautes études de l'Amérique latine, 1998.

Meunier, Jean-Pierre, "Connaître par l'image», Recherches en communication, $\mathrm{n}^{\circ}$ 10, 1998, p. 35-75.

Mezoued, Aniss, «Temps et récits urbains, essai méthodologique et éléments d'application au cas d'Alger", Urbia, Hors série n 1, 2013, p. 173-186.

Mezoued, Aniss, La mise en récit de l'urbanisme algérois, passé, présent, futur: À la recherche des conditions d'institution de l'espace public comme médiation et comme projet: Cas du tramway d'Alger, Presses universitaires de Louvain, 2015.

Michel, Aurélia, Denis, Éric et Rafael Soares Gonçalves, «Introduction : les enjeux du foncier urbain pour le développement - Nouveaux marchés et redistribution des responsabilités », Tiers Monde, nº206, 2011, p. 7-20.

Miraftab, Faranak, «Feminist Praxis, Citizenship and Informal Politics: Reflections on South Africa's Anti-Eviction Campaign", International Feminist Journal of Politics, vol. 8, $\mathrm{n}^{\circ}$ 2, 2006, p. 194-218.

Miraftab, Faranak, «Insurgent Planning: Situating Radical Planning in the Global South", Planning Theory, vol. 8, $\mathrm{n}^{\circ}$ 1, 2009, p. 32-50.

Miraftab, Faranak, Silver, Christopher et Victoria Beard, «Situating Contested Notions of Decentralized Planning in the Global South", dans Victoria BEARD, Faranak Mirafta B et Christopher Silver, Planning and Decentralization: Contested Spaces for Public Action in the Global South, Routledge, 2008, p. 1-18.

Mitchell, Timothy, Rule of Experts: Egypt, Techno-Politics, Modernity, University of California Press, 2002.

Monnet, Jérôme, «Mexique, Amérique latine et Amériques face à la métropolisation et à la mondialisation: Réflexions sur la permanence des cadres de l'analyse géographique », Historiens et géographes, $\mathrm{n}^{\circ} 374,2001$, p. 369-378.

Montulet, Bertrand et Vincent Kaufman, «Mobilités spatiales et fluidités sociales», dans Bertrand Montulet et Vincent Kaufman (dir.), Mobilités, fluidités... libertés?, Bruxelles, Publications des facultés universitaires SaintLouis, 2004, p. 9-21.

Morange, Marianne et Sylvie Fol, «Ville, néolibéralisation et justice», Justice Spatiale / Spatial Justice, $\mathrm{n}^{\circ}$ 6, 2014, <bit.ly/2oSEwGh>.

Morelle, Marie et Laetitia Laumonier, "Introduction au dossier», Revue Tiers Monde, vol. 1, $\mathrm{n}^{\circ} 185,2006$, p. 9-13.

Morelle, Marie, «La prison centrale de Yaoundé: l'espace au cœur d'un dispositif de pouvoir», Annales de géographie, $\mathrm{n}^{\circ}$ 3, 2013, p. 332-356. 
Murray, Martin J. et Garth A. Myers (dir.), Cities in Contemporary Africa, New York, Palgrave Macmillan, 2006.

Myers, Garth A., African Cities. Alternative Visions of Urban Theory and Practice, Londres \& New York, ZED Books, 2011.

NAUdeT, Jean-David, Trouver des problèmes aux solutions: vingt ans d'aide au Sahel, Paris, OCDE, 1999.

Navez-Bouchanine, Françoise et Licia Valladares, «Éditorial », Espaces et sociétés, vol. $4, \mathrm{n}^{\mathrm{0}} 131,2007$, p. 9-13.

Navez-Bouchanine, Françoise (dir.), La fragmentation en question: des villes entre fragmentation spatiale et fragmentation sociale?, Paris, L'Harmattan, 2002.

Navez-Bouchanine, Françoise (dir.), Effets sociaux des politiques urbaines, l'entre-deux des politiques institutionnelles et des dynamiques sociales, Paris, Karthala, 2012.

Newman, Peter et Jeffrey Kenworthy, Sustainability and Cities: Overcoming Automobile Dependence, Island Press, Washington D.C., 1999.

Odin, Françoise et Christian Thuderoz (dir.), Des mondes bricolés: arts et sciences à l'épreuve du bricolage, Lausanne, Presses polytechniques et universitaires romandes, 2011.

Oldfield, Sophie et Kristian STOKке, «Building Unity in Diversity: Social Movement Activism in the Western Cape Anti-Eviction Campaign", dans Richard BALLARD et al., Globalisation, Marginalisation and New Social Movements, Durban, University of KwaZulu-Natal Press, 2006, p. 25-49.

OldField, Sophie, "Negotiating Society and Identity in Urban Spaces of the South", dans Susan Parnell et Sophie Oldfield (dir.), The Routledge Handbook on Cities of the Global South, Routledge, 2014, p. 339-340.

Olivier De Sardan, Jean-Pierre, Anthropologie et développement. Essai en socioanthropologie du changement social, Marseille-Paris, APAD-Karthala, 1995.

Olivier De SARdan, Jean-Pierre, La rigueur du qualitatif: Les contraintes empiriques de l'interprétation socio-anthropologique, Louvain-la-Neuve, Bruylant Academia, 2008.

Osmont, Annick, La Banque mondiale et les villes: du développement à l'ajustement, Paris, Karthala, 1995.

Ottaway, Marina, Democracy Challenged: The Rise of Semi-Authoritarianism, Washington D.C., Carnegie Endowment for International Peace, 2003.

Panerai, Philippe et David Mangin, Proyectar la ciudad, Madrid, Celeste Ediciones, 2002.

PAQuot, Thierry, L'espace public, La Découverte, Paris, 2009.

PARnell, Susan et Jennifer Robinson, «(Re)theorizing Cities from the Global South: Looking Beyond Neoliberalism», Urban Geography, vol. 33, 2012, p. 593-617.

Patel, Sujata, "Is there a "South" Perspective to Urban Studies?», dans Susan Parnell et Sophie Oldfield (dir.), The Routledge Handbook on Cities of the Global South, Routledge, 2014, p. 37-47.

Peemans, Jean-Phillipe, "Développement, identités culturelles, villes et territoires: quelques aspects Nord-Sud» dans Bernard Declève et Dominique Нibo, 
Développement territorial et mutations culturelles, Presses universitaires de Louvain, 2000.

Peemans, Jean-Phillipe, "The many stakes and challenges of another look at the informal sector", dans Isabelle HILLENKAMP et al. (dir.), Securing Livelihoods, Informal Economy Practices and Institutions, Oxford University Press, 2013.

Pereira, Elson, Manoel et Mathieu Perrin, «Le droit à la ville: Cheminements géographique et épistémologique (France - Brésil - International) ", L'Information géographique, vol. 75, 2011, p. 15-36.

Peyronnie, Karinne et al., "Analyse des thèses portant sur les villes du Sud et soutenues en France en 1994 et 2011, bilan prospectif pour la recherche et la formation en coopération urbaine», Paris, Groupement d'intérêt scientifique pour l'étude de la mondialisation et du développement (GEMDEV), 2013.

Pierre Louis, Liliane et Léandre Guigma, «La contribution du Burkina Faso au Programme d'amélioration des bidonvilles mené par ONU-Habitat: Une réflexion sur les espoirs puis désillusions suscitées chez la population », Communication au séminaire REHAL «Vulnérabilités résidentielles: Comment enquêter sur la vulnérabilité résidentielle? Échanges d'expériences », Paris, 7 février 2014.

Pieterse, Edgar, Exploratory Notes on African Urbanism, Cape Town, African Centre for Cities, University of Cape Town, 2009.

Pinson, Daniel, «L'Urbanisme: une discipline indisciplinée?», Futures, vol. 36, nº 4, 2004, p. 503-513.

Polèse, Mario, Wolfe, Jeanne et Sylvain Lefebvre, L'urbanisation des pays en développement, Paris, Economica, 1995.

RaIson, Jean-Paul, «Nommer, c'est créer un peu: De «Tiers-Monde» à «Tropicalisme» : les avatars d'un vocabulaire ", Autrepart, nº 41, 2007, p. 57-68.

Rammstedt, Otthein, «L'étranger de Georges Simmel», Revue des sciences sociales de la France de l'Est, 1994 p. 146-153.

RaOul, Bruno, «Un travail d'enquête à l'épreuve du terrain ou "l'expérience de terrain" comme relation en tension ", Études de communication, $\mathrm{n}^{\circ} 25,2002$, <http:// edc.revues.org >.

RENARD, Vincent, «Recherche urbaine et coopération avec les pays en développement: À la recherche d'un nouveau paradigme», dans Annick Osmont et Charles Goldblum, Villes et citadins dans la mondialisation, Paris, Karthala-GEMDEV, 2003, p. 237-245.

Ribeiro de Souza, Amélia et Valeria Cartes Leal, Les chemins de l'école: déplacements scolaires dans la Région de Bruxelles-Capitale, Louvain-la-Neuve, Presses universitaires de Louvain, "Territoires et développements durables», 2005.

RigG, Jonathan, An Everyday Geography of the Global South, Routledge, 2007.

Rivière D’Arc, Hélène et Maurizio Memoli (dir.), Le pari urbain en Amérique latine: Vivre dans le centre des villes, Paris, Armand Colin, 2006.

Robinson, Jennifer, "Cities in a World of Cities: The Comparative Gesture», International Journal of Urban and Regional Research, vol. 35, $\mathrm{n}^{\circ}$ 1, 2011a, p. 1-23.

RoBinson, Jennifer, "Global and World Cities: a View from off The Map», International Journal of Urban and Regional Research, vol. 26, $\mathrm{n}^{\circ}$ 3, 2002, p. 531-554. 
Robinson, Jennifer, «New Geographies of Theorizing the Urban: Putting Comparison to Work for Global Urban Studies", dans Susan PARnell et Sophie Oldfield (dir.), The Routledge Handbook on Cities of the Global South, Routledge, 2014, p. 57-70.

Robinson, Jennifer, «The Spaces of Circulating Knowledge: City Strategies and Global Urban Governmentality", dans Eugene McCann et Kevin Ward (dir.), Mobile Urbanism, University of Minnesota Press, 2011b, p. 15-39.

Robinson, Jennifer, Ordinary cities: between modernity and development, Routledge, 2006.

Rochefort, Michel, Le Défi urbain dans les pays du Sud, Paris, L'Harmattan, 2000.

Roshan, Samara, Tony, He, Shenjing et Guo Chen, Locating Right to the City in the Global South, Routledge, 2013.

Rотн, Alfred, La Nouvelle École, Zurich, Éditions Girsberger, 1966.

Roy, Ananya et Aihwa ONG, Worlding Cities: Asian Experiments and the Art of Being Global, John Wiley \& Sons, 2011.

Roy, Ananya, "Slumdog Cities: Rethinking Subaltern Urbanism», International Journal of Urban and Regional Research, vol. 35, $\mathrm{n}^{\circ}$ 2, 2011, p. 223-238.

Roy, Ananya, «Urban Informality: Toward an Epistemology of Planning», Journal of the American Planning Association, vol. 71, $\mathrm{n}^{\circ}$ 2, 2005, p. 147-158.

Roy, Ananya, "Who's Afraid of Postcolonial Theory?", International Journal of Urban and Regional Research, vol. 40, $\mathrm{n}^{\circ} 1,2015$, p. 200-209.

Roy, Ananya, "Worlding the South. Toward a Post-Colonial Urban Theory", dans Susan Parnell et Sophie Oldfield (dir.), The Routledge Handbook on Cities of the Global South, Routledge, 2014, p. 9-27.

Roy, Ananya, "Strangely Familiar: Planning and the Worlds of Insurgence and Informality», Planning Theory, vol. 1, n 8, 2009b, p. 7-11.

Saint-Lary-MaÏga, Maud, Boulllon, Florence, Frésia, Marion et Virginie Tallio (dir.), «Terrains sensibles: Expériences actuelles de l'anthropologie», Cahiers d'études africaines, $\mathrm{n}^{\circ}$ 191, 2008, <http://etudesafricaines.revues.org>.

Saint-Martin, Arnaud, "Les bricolages sociologiques: Du pluralisme en sciences sociales", Esprit critique, vol. 4, n 2, 2002.

SALAT, Serge, Les villes et les formes: Sur l'urbanisme durable, Paris, Hermann-CSTB, 2011.

SANDERCock, Leonie, Making the Invisible Visible: A Multicultural Planning History, University of California Press, 1998.

SAndercock, Leonie Jean-Pierre, Towards Cosmopolis: Planning for Multicultural Cities, Chichester, Wiley \& Sons, 1998.

Sassen, Saskia, La ville globale: New York, Londres, Tokyo, Paris, Descartes \& Cie, 2006.

Scarwell, Helga-Jane, Leduce, Divya et Du Tran Dinh, «Hanoi, une ville vietnamienne en transition urbaine vers le développement durable», dans Isabelle Hajek, Philippe Hamman et Jean-Pierre Lévy (dir), Villes du Nord, villes du Sud: la durabilité, entre prêt à penser et opérationnalité, Presses universitaires du Septentrion, 2015, p. 33-58. 
Scherrer, Franck, «Le champ de la recherche en urbanisme existe-t-il? Quelques repères pour la relève», Urbia, Hors série $\mathrm{n}^{\circ} 1,2013$.

SCHERRER, Franck, «Le contrepoint des études urbaines et de l'urbanisme, ou comment se détacher de l'évidence de leur utilité sociale», Tracés, vol. 10, 2010, p. 187-195.

SснӧN, Donald A., «Knowing-in-Action: The New Scholarship Requires a New Epistemology", Change: The Magazine of Higher Learning, vol. 27, $\mathrm{n}^{\circ}$ 6, 1995, p. 27-34.

SeCCHI, Bernardo, La ville du vingtième siècle, Paris, Recherches, 2009.

Secchi, Bernardo, Première leçon d'urbanisme, Marseille, Parenthèses, 2006.

Segbers, Klaus (dir.), The Making of Global City Regions: Johannesburg, Mumbai/ Bombay, Sao Paulo, and Shanghai, Baltimore, The John Hopkins University Press, 2007.

Shatkin, Gavin, "Contesting the Indian City: Global Visions and The Politics of the Local», International Journal of Urban and Regional Research, vol. 38, $\mathrm{n}^{\circ}$ 1, 2014, p. 1-13.

Shatkin, Gavin, "Coping with Actually Existing Urbanisms: The Real Politics of Planning in the Global Era», Planning Theory, vol. 10, $\mathrm{n}^{\circ}$ 1, 2011, p. 79-87.

Shatkin, Gavin, "Global Cities of the South: Emerging Perspectives on Growth and Inequality», Cities, vol. 24, $\mathrm{n}^{\circ}$ 1, 2007, p. 1-15.

Shatkin, Gavin, "Planning Privatopolis: Representation and Contestation in the Development of Urban Integrated Mega-Projects», dans Ananya Roy et Aihwa ONG, Worlding Cities: Asian Experiments and the Art of Being Global, Wiley \& Sons, 2011.

Shatkin, Gavin, "Coping with actually existing urbanisms: The real politics of planning in the global era", Planning Theory, vol. 10, p. 79-87, 2011.

ShePpard, Eric et al., A World of Difference: Encountering and Contesting Development, New York, Guilford Press, 2009.

Sheppard, Eric, "Globalizing Capitalism and Southern Urbanization », dans Susan Parnell et Sophie Oldfield (dir.), The Routledge Handbook on Cities of the Global South, Routledge, 2014, p. 143-154.

Sheppard, Eric, Leitner, Helga et Anant Maringanti, «Provincializing Global Urbanism: a Manifesto ", Urban Geography, vol. 34, n 7, 2013, p. 893-900.

Sierra, Alexis et Jérôme TAdié, «La ville face à ses marges", Autrepart, $\mathrm{n}^{\circ}$ 45, 2008, p. 3-13.

Simmel, Georges, "Digression sur l'étranger», 1908, dans Yves Grafmeyer et Isaac Joseph, L'école de Chicago, Paris, Aubier, 1984, p. 53-59.

Simon, David, «Rethinking (post) Modernism, Postcolonialism, and Posttraditionalism: South-North Perspectives ", Environment and Planning D: Society and Space, vol. 16, n' 2, 1998, p. 219-245.

Simon, David, «The World City Hypothesis: Reflections from The Periphery», dans Paul L. Knox et Peter J. TAYlor (dir.), World Cities in a World System, 1995, p. 132-155. 
Simone, Abdoumaliq, The Social Infrastructures of City Life in Contemporary Africa, The Nordic Africa Institute, Uppsala, 2010.

Simone, Abdoumaliq, City Life from Jakarta to Dakar: Movements at the Crossroads, Routledge, 2010.

Simone, Abdoumaliq, For the City Yet to Come: Changing African Life in Four Cities, Durham, Duke University Press, 2004.

Simonneau, Claire, "Gérer la ville au Bénin: La mise en œuvre du Registre foncier urbain à Cotonou, Porto-Novo et Bohicon», thèse en aménagement, Université de Montréal, 2015.

Singly (DE), François, Le questionnaire, Paris, Armand Colin, 2008.

Slater, Tom, «Planetary Rent Gaps», Antipode, 2015, <bit.ly/203JbrF>.

Sмiтн, Michael P., Transnational Urbanism: Locating Globalization, Wiley-Blackwell, 2000.

Smith, Neil, «New Globalism, New Urbanism: Gentrification as Global Urban Strategy", Antipode, vol. 34, n 3, 2002, p. 427-450.

Söderström Ola et AL., Critical Mobilities, Routledge, 2013.

Söderström, Ola, Cities in Relations: Trajectories of Urban Development in Hanoi and Ouagadougou, Malden, Wiley Blackwell, 2014.

SojA, Edward W., Postmodern Geographies: The Reassertion of Space in Critical Social Theory, New York, Verso, 1989. (vérifier la date qui n'est pas la même que celle écrite à la p. 28).

Sory, Issa, «Ouaga la belle!» Gestion des déchets solides à Ouagadougou: enjeux politiques, jeux d'acteurs et inégalités environnementales, Thèse de Géographie, Université Paris 1 Panthéon-Sorbonne, 2013.

Souami, Taoufik et Éric Verdeil (dir.), Concevoir et gérer les villes: milieux d'urbanistes du sud de la Méditerranée, Paris, Anthropos, 2006.

SouAmi, Taoufik, "Liens interpersonnels et circulation des idées en urbanisme: L'exemple des interventions de l'IAURIF au Caire et à Beyrouth ", Géocarrefour, $\mathrm{n}^{\circ} 88,2005$, p. 237-247.

Staeneli, Lynn et Don Mitchell, The People's Property? Power, Politics and the Public, New York, Routledge, 2007.

STREN, Richard, «Old wine in new bottles? An overview of Africa's urban problems and the "urban management" approach to dealing with them ", Environment and Urbanization, vol. 3, 1991, p. 9-22.

Supiot, Alain, «Communiquer ou se comprendre? La question du régime linguistique de la recherche en sciences humaines et sociales», Trivium, ${ }^{\circ}{ }^{15}, 2013$.

TALL, Serigne Mansour, Investir dans la ville africaine: les émigrés et l'habitat à Dakar, Paris, Karthala, 2009.

Troin, Jean-François, Les métropoles des «Sud», Paris, Ellipses, 2000.

UN-Habitat, State of the World's Cities 2010/2011: Bridging The Urban Divide, Nairobi, UN-Habitat, 2011.

Urry, John, Mobilities, Malden, Polity, 2007. 
Van Damme, Stéphane, «Une histoire des savoirs urbains est-elle possible? ", Metropolitiques.eu, $2013<$ bit.ly/2n5L5c8>.

VARrel, Aurélie, «L'émergence d'un marché transnational de l'immobilier indien ", Autrepart, $\mathrm{n}^{\circ}$ 67-68, 2013, p. 233-246.

VERDEIL, Éric, «Expertises nomades au Sud. Éclairages sur la circulation des modèles urbains », Géocarrefour, vol. 8o, n 3, 2005, p. 165-169.

VerdeIL, Éric, «Recension de Métropoles XXL: repenser le développement urbain à partir des réseaux d'infrastructure?», Métropolitiques.eu, 2012, <www.metropolitiques.eu/Metropoles-XXL-repenser-le.html>.

Véron, Jacques, L’urbanisation du monde, Paris, La Découverte, 2006.

Vigano, Paola, Les territoires de l'urbanisme: Le projet comme producteur de connaissance, Paris, MétisPresses, 2012.

WaEchter-Larrondo, Virginie, «Plaidoyer pour le bricolage et l'enracinement des méthodes d'enquête dans le terrain: l'exemple d'une recherche sur le changement dans les services publics locaux", Bulletin de méthodologie sociologique, $\mathrm{n}^{\circ} 88$, 2005, p. 31-6o.

WARD, Kevin, «Editorial Toward a Comparative (Re)turn in Urban Studies? Some Reflections", Urban Geography, vol. 29, n 5, 2008, p. 405-410.

Watson, Sophie, «Spaces of Difference: Challenging Urban Divisions from the North to the South», dans Susan Parnell et Sophie Oldfield (dir.), The Routledge Handbook on Cities of the Global South, Routledge, 2014, p. 385-395.

Watson, Vanessa, «Seeing from the South: Refocusing Urban Planning on the Globe's Central Urban Issues », Urban Studies, vol. 46, n 11, 2009, p. 2259-2275.

Watson, Vanessa, "The Allure of "Smart City" Rhetoric India and Africa», Dialogues in Human Geography, vol. 5, n 1, 2015, p. 36-39.

Watson, Vanessa, «The Usefulness of Normative Planning Theories in the Context of Sub-Saharan Africa», Planning Theory, vol. 1, $\mathrm{n}^{0}$ 1, 2002, p. 27-52.

YeOH, Brenda S. A., «Postcolonial Cities », Progress in Human Geography, vol. 25, $\mathrm{n}^{\circ}$, 2001, p. 456-468. 



\section{Table des matières}

Avant-propos

Repenser la place des villes non occidentales

dans les recherches urbaines

Gabriel Fauveaud

\section{PREMIÈRE PARTIE \\ L'ESPACE PUBLIC COMME MÉdiation}

CHAPITRE 1

L'espace public comme fabricant de liens

Aniss M. Mezoued

CHAPITRE 2

L'espace public et le patrimoine

Pauline Bosredon

CHAPITRE 3

Les revers du budget participatif

Cécilia Montoya Antich

CHAPITRE 4

L'école coproductrice de la ville et du projet de société

Valeria Cartes Leal 


\title{
DEUXIÈME PARTIE \\ LA FABRICATION URBAINE \\ AU PRISME DES JEUX D'ÉCHELLES
}

CHAPITRE 5

L'échelle dans la définition de l'urbain et de l'urbanité

Mauricio Anaya

CHAPITRE 6

De la planification stratégique au droit à la ville

Issa Sory

CHAPITRE 7

Mobilité, échelles et chemins

Bianca De Marchi Moyano

\author{
TROISIÈME PARTIE \\ LES ENJEUX RÉFLEXIFS \\ ET MÉTHODOLOGIQUES DU TERRAIN
}

CHAPITRE 8

Les outils d'urbanisme, entre modernité et développement

Claire Simonneau

CHAPITRE 9

Un nécessaire bricolage méthodologique

Divya Leduca

CHAPITRE 10

Les identités du chercheur

Léandre Guigma

Les auteurs

Bibliographie 
1939: l'alliance de la dernière chance. Une réinterprétation des origines de la Seconde Guerre mondiale • Michael J. Carley

L'Afrique du Sud. Entre émergence et responsabilité • Moda Dieng

L'aide au conditionnel. La contrepartie dans les mesures envers les personnes sans emploi en Europe et en Amérique du Nord • Gérard Boismenu, Pascale Dufour et Alain Noël

L'aide canadienne au développement • François Audet, Marie-Eve Desrosiers et Stéphane Roussel

Amerindia. Essais d'ethnohistoire autochtone • Roland Viau

L'analyse des politiques publiques • Luc Bernier, Guy Lachapelle et Stéphane Paquin

Anatomie de la prison contemporaine. Nouvelle édition • Guy Lemire et Marion Vacheret

L’année politique au Québec. 1997-1998 • Robert Boily

Apprendre en situation d'autodidaxie $\bullet$ Nicole Anne Tremblay

Approches et pratiques en évaluation de programmes. Nouvelle édition revue et augmentée $\bullet$ Christian Dagenais, Valéry Ridde

Architecture de paysage du Canada $\bullet$ Ron Williams

L’armée canadienne vous parle. Communication et propagande gouvernementales • Isabelle Gusse

Les Autochtones et le Québec. Des premiers contacts au Plan Nord• Alain Beaulieu, Stéphan Gervais et Martin Papillon

Aux origines sociales de l'État-providence $\bullet$ Dominique Marshall

Barcelone ou comment refaire une ville $\bullet$ Béatrice Sokoloff

La Beauce Inc. Capital social et capitalisme régional • Jacques Palard

De la beauté comme violence. L'esthétique du fascisme français, 1919-1939• Michel Lacroix

Le bon sens à la scandinave. Les inégalités sociales de santé • Dominique Côté et Marie-France Raynault

La bourgeoisie industrielle au Québec • Arnaud Sales

Le campus. Le patrimoine architectural et paysager de l'Université de Montréal / The Architectural and Landscape Heritage • Christina Cameron, Claudine Déom et Nicole Valois

Le Canada et l'Arctique • Franklyn Griffith, Rob Huebert et P. Whitney Lackenbauer

Le Canadien de Montréal. Une légende repensée • Audrey Laurin-Lamothe et Nicolas Moreau (dir.)

Charte canadienne et droits linguistiques. Pour en finir avec les mythes • Frédéric Bérard

La Chine imaginaire. Les Chinois vus par les Occidentaux, de Marco Polo à nos jours • Jonathan D. Spence

Le comportement électoral des Québécois • Éric Bélanger et Richard Nadeau

Consolidation de la paix et fragilité étatique. L'ONU en République Centrafricaine• Jocelyn Coulon et Damien Larramendy, Marie-Joëlle Zahar (coll.)

Continuité et rupture. Les sciences sociales au Québec • Georges-Henri Lévesque (dir.)

Contre la réforme. La dérive idéologique du système d'éducation québécois • Normand Baillargeon 
Converties à l'islam. Parcours de femmes au Québec et en France • Géraldine Mossière

Croissance et développement de l'enfant québécois de la naissance à six ans • Arto Demirjian

Le débat linguistique au Québec • Donat J. Taddeo et Raymond C. Taras

Débattre pour transformer l'action urbaine. Planification urbaine et développement durable à Grenoble, Lyon et Montréal • Michel Gariépy, Mario Gauthier, Franck Scherrer, Florence Paulhiac Scherrer et Lila Combe

Décentralisation, politiques publiques et relations de pouvoir • Vincent Lemieux

Les défis du nationalisme moderne. Québec, Catalogne, Écosse • Michael Keating

La délinquance sexuelle des mineurs. Approches cliniques • Monique Tardif, Martine Jacob, Robert Quenneville et Jean Proulx (dir.)

Démocratie médiatique et représentation politique • Denis Monière

La démographie québécoise. Enjeux du $\mathrm{xx}^{e}$ siècle $・$ Victor Piché et Céline Le Bourdais (dir.)

Le désengagement de l'État providence $\bullet$ Nicole F. Bernier

Des idées reçues en santé mondiale • Valéry Riddle et Fatoumata Ouattara

Développement durable et participation publique. De la contestation écologiste aux défis de la gouvernance • Corinne Gendron et Jean-Guy Vaillancourt (dir.)

La différenciation sociale. Modèles et processus $•$ Danielle Juteau (dir.)

Discrimination et accès aux soins en Guyane française • Estelle Carde

Un drame de la Deuxième Guerre. Le sort de la minorité japonaise aux États-Unis et au Canada • Greg Robinson

Douceville en Québec. La modernisation d'une tradition • Colette Moreux

Drogue et criminalité. Une relation complexe (Troisième édition revue et augmentée) • Serge Brochu, Natacha Brunelle et Chantal Plourde

Du pain ou du sang. Les travailleurs irlandais et le canal Beauharnois • Roland Viau

L'économie circulaire. Une transition incontournable • Sébastien Sauvé, Daniel Normandin et Mélanie McDonald (dir.)

L'éducation aux médias à l'ère numérique. Entre fondations et renouvellement • Normand Landry et Anne-Sophie Letellier (dir.)

Éducation en milieu urbain • Manuel Crespo et Claude Lessard (dir.)

De l'éducation spécialisée • Maurice Capul et Michel Lemay

Éduquer avant l'école. L'intervention préscolaire en milieux défavorisés et pluriethniques • François Victor Tochon (dir.)

Emploi et salaire. Deuxième édition revue et augmentée • Jean-Michel Cousineau

Énergie et fédéralisme au Canada $•$ Michel Duquette

Les enfants qu'on n'a plus au Québec • Jacques Henripin, Paul-Marie Huot, Évelyne Lapierre-Adamcyk et Nicole Marcil-Gratton

L'énigme haïtienne. Échec de l'État moderne en Haïti • Sauveur Pierre Étienne

L'état des citoyennetés. En Europe et dans les Amériques • Jane Jenson,

Bérengère Marques-Pereira et Éric Remacle (dir.)

L'éthique et le droit. Face aux nouvelles technologies biomédicales • Guy Bourgeault

L'ethnicité et ses frontières • Danielle Juteau

Et si nous dansions? Pour une politique du bien commun au Canada • Charles Blattberg

L'Europe qui se fait. Regards croisés sur un parcours inachevé • Gérard Boismenu et Isabelle Petit (dir.) 
Les excuses dans la diplomatie américaine. Pour une approche pluraliste des relations internationales $•$ Jérémie Cornut

La famille et les troubles émotionnels des jeunes • Claude Villeneuve

Le fédéralisme canadien contemporain. Fondements, traditions, institutions • Alain-G. Gagnon (dir.)

Fédéralisme et cours suprêmes. Federalism and Supreme Courts • Edmond Orban et collaborateurs

Femmes philanthropes. Catholiques, protestantes et juives dans les organisations caritatives au Québec • Yolande Cohen

France-Canada-Québec. 400 ans de relations d'exception $\bullet$ Serge Joyal et Paul-André Linteau (dir.)

La France depuis de Gaulle. La Ve République en perspective • Marc Chevrier et Isabelle Gusse (dir.)

La gauche et la droite. Un débat sans frontières • Alain Noël et Jean-Philippe Thérien

Gérer les catastrophes. L'incertitude à apprivoiser • Hélène Denis

Gestion des ressources humaines. Du modèle traditionnel au modèle renouvelé • Gilles Guérin et Thierry Wils

La gestion écologique des déchets • Jean-Guy Vaillancourt, Michel Séguin, Louis Maheu et Liliane Cotnoir, collaborateur: Pierre Hamel

Gouvernance et planification collaborative. Cinq métropoles canadiennes • Michel Gariépy et Olivier Roy-Baillargeon (dir.)

Les grandes universités de recherche. Institutions autonomes dans un environnement concurrentiel $\bullet$ Robert Lacroix et Louis Maheu

Grands projets et innovations technologiques au Canada • Philippe Faucher (dir.)

Les grands projets urbains. Territoires, acteurs et stratégies • Michel Max Raynaud, Paul Lewis et Michel Hubert

Grands Seigneurs et multinationales. L'économie politique de l'éthanol au Brésil • Michel Duquette

Les grèves au Canada: causes et conséquences • Robert Lacroix

Une guerre sourde. L'émergence de l'Union soviétique et les puissances occidentales • Michael Jabara Carley

Guide méthodologique universitaire. Un programme en 12 semaines • Aude Jimenez et Jamal-Eddine Tadlaoui

Gunnar Myrdal et son œuvre • Gilles Dostaler, Diane Éthier et Laurent Lepage (dir.)

Guide de l'architecture contemporaine de Montréal • Nancy Dunton et Helen Malkin

Guy Rocher. Le savant et le politique • Violaine Lemay et Karim Benyekhlef (dir.)

Images et sociétés. Le progrès, les médias et la guerre • Catherine Saouter

Immigration et diversité à l'école. Le débat québécois dans une perspective comparative $\bullet$ Marie Mc Andrew

L'Inde dans tous ses états • Guy Taillefer

L'Inde et ses avatars. Pluralité d'une puissance • Serge Granger, Christophe Jaffrelot, Karine Bates et Mathieu Boisvert

Les inégalités sociales de santé au Québec • Katherine Frohlich, Maria

De Koninck, Andrée Demers et Paul Bernard (dir.)

Initiation à la recherche en géographie. Aménagement, développement territorial, environnement • Hervé Gumuchian et Claude Marois

L'intégration des familles d'origine immigrante. Les enjeux sociosanitaires

et scolaires • Fasal Kanouté et Gina Lafortune (dir.) 
L'intégration des immigrants. Cinquante ans d'action publique locale • Aude-Claire Fourot

L'interculturel au Québec. Rencontres historiques et enjeux politiques • Bob W. White et Lomomba Emongo

Interprétations radicales $\bullet$ Daniel Laurier

Introduction à la politique africaine. Deuxième édition revue et augmentée • Mamoudou Gazibo

Introduction à la victimologie • Jo-Anne Wemmers

Introduction aux relations internationales. Quatrième édition • Diane Éthier

Le Japon au travail • Bernard Bernier avec la collaboration de Vincent Mirza

Le Japon contemporain. Une économie nationale, une économie morale •

Bernard Bernier

Je suis un lieu • Jacynthe Tremblay

Jeux de mémoire • Bruno Roy et Paul Zumthor (dir.)

Les Juifs du Maghreb. Diasporas contemporaines • Jean-Claude Lasry et Claude Tapia

Maintenir la paix en zones postconflit. Les nouveaux visages de la police•

Samuel Tanner et Benoit Dupont (dir.)

Les majorités fragiles et l'éducation. Belgique, Catalogne, Irlande du Nord, Québec • Marie Mc Andrew

Maurice Sand. Une cuvre et son brisant au $19^{e}$ siècle • Lise Bissonnette

Méchante langue. La légitimité linguistique du français parlé au Québec •

Chantal Bouchard

Médecine(s) et santé. Une petite histoire globale $\bullet 19^{\mathrm{e}}$ et $2 \mathrm{O}^{\mathrm{e}}$ siècles $\bullet$ Laurence Monnais

Méthodes de recherche pour l'éducation • Jean-Marie Van der Maren

Micro-économie. Exercices et corrigés • François Leroux

Milton Friedman et son cuvre • Marc Lavoie et Mario Seccareccia (dir.)

Les meurtriers sexuels. Analyse comparative et nouvelles perspectives • Jean Proulx,

Maurice Cusson, Eric Beauregard et Alexandre Nicole (dir.)

Un modèle québécois? Gouvernance et participation dans la gestion publique •

Pierre Hamel et Bernard Jouve

Les MOOCs. Cours en ligne et transformations des universités • Pablo Achard

Les migrations internationales contemporaines. Une dynamique complexe

au cour de la globalisation • François Crépeau, Delphine Nakache

et Idil Atak (dir.)

Montréal en paysages • Philippe Poullaouec-Gonidec et Sylvain Paquette

Montréal et Toronto. Villes intérieures • Michel Boisvert

Monuments intellectuels de la Nouvelle-France et du Québec ancien. Aux origines d'une tradition culturelle $\bullet$ Claude Corbo (dir.)

Les mouvements sociaux • Antimo L. Farro

Naissance d'une population. Les Français établis au Canada au XVII siècle • Hubert Charbonneau (dir.)

Nouvelles valeurs et gouvernance au Canada $•$ Neil Nevitte (dir.)

Un nouvel ordre mondial made in China? • Mamoudou Gazibo et Roromme Chantal

L'ordre et la fête • Frédéric Diaz

Où va le modèle suédois? État-providence et protection sociale •

Lionel-Henri Groulx 
Les paradoxes de la démocratisation en Afrique. Analyse institutionnelle et stratégique $\bullet$ Mamoudou Gazibo

Parcours éducatifs. Construction de soi et transformation sociale • Paul Bélanger

Paysages ruraux. Méthodes d'état des lieux et de diagnostic • Gérald Domon et Julie Ruiz

La pauvreté. Quatre modèles sociaux en perspective • Sylvain Lefèvre, Gérard Boismenu et Pascale Dufour

Penser l'international. Perspectives et contributions des sciences sociales • François Crépeau et Jean-Philippe Thérien (dir.)

Philosophies de la connaissance • Robet Nadeau (dir.)

Les pièges de l'austérité • Pierre Paquette et Mario Seccareccia

Planifier et évaluer l'action sociale • Ricardo Zúñiga

Plurilinguisme et pluriculturalisme. Des modèles officiels dans le monde • Gillian Lane-Mercier, Denise Merkle et jane Koustas

Politique internationale et défense au Canada et au Québec • Kim Richard Nossal, Stéphane Roussel et Stéphane Paquin

La politique comparée. Fondements, enjeux et approches théoriques • Mamoudou Gazibo et Jane Jenson

La politique économique du développement et les modèles d'équilibre général calculable • Bernard Decaluwé, André Martens et Luc Savard

La politique en questions $•$ Professeurs de science politique de l'Université de Montréal

La politique internationale en questions $•$ Professeurs de science politique de l'Université de Montréal

Les populations amérindiennes et inuit du Canada. Aperçu démographique • Louise Normandeau et Victor Piché (dir.)

La population du Québec d'hier à demain • Jacques Henripin et Yves Martin (dir.)

Pour comprendre le nationalisme, au Québec et ailleurs • Denis Monière

Pour un nouvel art de vivre. Entretiens sur la vie, la santé, l'éthique biomédicale et l'éducation • Daisaku Ikeda, René Simard et Guy Bourgeault

Pour un regard-monde $•$ Armand Mattelart

Pratiques innovantes auprès des jeunes en difficulté • Denis Lafortune,

Marie-Marthe Cousineau et Claudia Tremblay (dir.)

Prévention des toxicomanies. Aspects théoriques et méthodologiques • Pierre Brisson

Profession démographe $\bullet$ Victor Piché

Profession géographe • Rodolphe De Koninck

Profession sinologue $\bullet$ Charles Le Blanc

Profession sociologue $\bullet$ Marcel Fournier

Profession urbaniste $\bullet$ Gérard Beaudet

Protection de l'enfant: échec? • Alice Parizeau

Protection sociale et fédéralisme. L'Europe dans le miroir de l'Amérique du Nord • Bruno Théret

La psychocriminologie. Apports psychanalytiques et applications cliniques • Dianne Casoni et Louis Brunet

La psychologie des adolescents $\bullet$ Michael Claes

Les Québécois aux urnes. Les partis, les médias et les citoyens en campagne • Éric Bélanger, Frédérick Bastien, François Gélineau 
Quelle justice internationale au Proche Orient? Le cas du Tribunal spécial pour le Liban • Fady Fadel et Cynthia Eid

Questions de criminologie • Jean Poupart, Denis Lafortune et Samuel Tanner (dir.) Qui a peur de l'État? Le Canada dans un monde aux stuctures polycentriques de pouvoir • Gordon Smith et Daniel Wolfish (dir.)

Radioscopie de l'information télévisée au Canada • Denis Monière et Julie Fortier Les raisons fortes. Nature et signification de l'appui à la souveraineté du Québec • Gilles Gagné et Simon Langlois

La recomposition du politique $\bullet$ Louis Maheu et Arnaud Sales (dir.)

La réforme du droit de la famille. Une comparaison Sénégal-Maroc • Marième N'Diaye

Les relations ethniques en question. Ce qui a changé depuis le 11 septembre • Jean Renaud, Linda Pietrantonio et Guy Bourgeault (dir.)

Le régime monétaire canadien. Institutions, théories et politiques $\bullet$ Bernard Élie La résolution de conflits. Guide d'implantation et de pratiques • Nina Admo Les sciences sociales de l'environnement. Analyses et pratiques • Brigitte Dumas, Camille Raymond et Jean-Guy Vaillancourt (dir.)

Les scientifiques et la paix. La communauté scientifique internationale au cours des années 20 • Brigitte Schroeder-Gudehus

Sexes et pouvoir. La construction sociale des corps et des émotions • Marc Préjean Les sextants de Pékin • Joanna Waley-Cohen

Signé Papineau. La correspondance d'un exilé • Yvan Lamonde

Socialisation et communication dans les jeux vidéo • Charles Perraton, Magda Fusaro et Maude Bonenfant (dir.)

La societé efficiente. Pourquoi fait-il si bon vivre au Canada?• Joseph Heath. Traduit de l'anglais par Jean Chapdelaine Gagnon

La sociocriminologie $\bullet$ Stéphane Leman-Langlois

La sociologie des populations • Victor Piché et Hubert Gérard (dir.)

Sociologie et société québécoise. Présences de Guy Rocher • Céline Saint-Pierre et Jean-Philippe Warren (dir.)

Sphères de surveillance • Stéphane Leman-Langlois (dir.)

Stratégies de recherche en sciences sociales. Applications à la gérontologie • Richard Lefrançois

Le système de santé québécois. Un modèle en transformation • Clermont Bégin, Pierre Bergeron, Pierre-Gerlier Forest et Vincent Lemieux (dir.)

Le système politique américain • Michel Fortmann et Pierre Martin (dir.)

Terrorisme et anti-terrorisme au Canada • Stéphane Leman-Langlois et Jean-Paul Brodeur (dir.)

Théories de la politique étrangère américaine. Auteurs, concepts et approches • Charles-Philippe David (dir.)

La thèse. Un guide pour y entrer... et s'en sortir • Emmanuelle Bernheim et Pierre Noreau (dir.)

Le Tiers-Monde postcolonial. Espoirs et désenchantements • Maurice Demers, Patrick Dramé (dir.)

Tradition antique et tolérance moderne. Textes choisis et présentés par Philippe Despoix et Georges Leroux • Raymond Klibansky

Tradition et innovation en Chine et au Japon • Charles Le Blanc et Alain Rocher (dir.) 
Traité de criminologie empirique • Marc Le Blanc et Maurice Cusson (dir.)

Trajectoires de la neutralité • Valérie Amiraux, David Koussens (dir.)

Le travail au féminin. Analyse démographique de la discontinuité professionnelle des femmes au Canada • Marianne Kempeneers (dir.)

Trois espaces de protestation. France, Canada, Québec • Pascale Dufour

L'univers social des adolescents • Michel Claes

Le vert et le bleu. Identité québécoise et identité irlandaise au tournant du $\mathrm{xx}^{e}$ siècle • Simon Jolivet

Les Vietnamiens de Montréal • Louis-Jacques Dorais et Éric Richard

Les visages de la police. Pratiques et perceptions • Jean-Paul Brodeur

Vivre en famille au cour de la ville • Jean-Philippe Meloche et Juan Torres

$Z$ pour Zombies $•$ Bernard Perron, Antonio Dominguez Leiva et Samuel Archibald (dir.) 